\title{
Molecular Regulation of Fetal Brain Development in Pigs
}

\author{
A Thesis presented to \\ the Faculty of the Graduate School \\ University of Missouri-Columbia \\ Division of Animal Science \\ In partial Fulfillment \\ of the Requirements for the \\ Degree Master of Science \\ by \\ MONICA P. STRAWN \\ Dr. Susanta K. Behura, Thesis Supervisor
}

July 2021 
The undersigned, appointed by the Dean of the Graduate School, have examined the thesis entitled

\section{Molecular Regulation of Fetal Brain Development in Pigs}

Presented by Monica P. Strawn

A candidate for the degree of Master of Science,

And hereby certify that in their own opinion it is worthy of acceptance.

Dr. Susanta K. Behura

Dr. Timothy J. Safranski

Dr. Brenda T. Beerntsen 


\section{ACKNOWLEDGEMENTS}

I would like to express my sincere gratitude to my advisor, Dr. Behura, for taking me on as a young, naive graduate student that had no idea what I was fully getting myself into. He provided continued patience, enthusiasm, and immense knowledge as we navigated this together. My time here has been anything but typical, but I am honored to be Dr. Behura's first graduate student as we traversed this process together.

Thank you to Dr. Safranski and Dr. Beerntsen, for serving on my committee and giving encouragement, practical applications, and scientific knowledge. Dr. Safranski was invaluable in finding the holes in my study plan in terms of animal management and for that I am grateful. Dr. Beernsten was incredibly helpful in the editing and refining process of my writing.

A special thank you goes out to all my mentors that did not serve on my committee but were always there for me in the small ways. Depending on the day, that might have been a smile in the hallways, even though I could not see it behind the masks, or a confidant I could turn to when I had life questions and someone who was there to check on me periodically.

I also want to thank all my fellow scientists and graduate students that showed me so many procedures, tips, and tricks, and answered all my questions no matter how dumb they seemed. More importantly, I am thankful for the friendships I have gained.

Finally, thank you to my family and friends who have supported me in all my life endeavors, encouraged me to keep going, shaping me into who I am today and stuck with me through the stressful times. 


\section{TABLE OF CONTENTS}

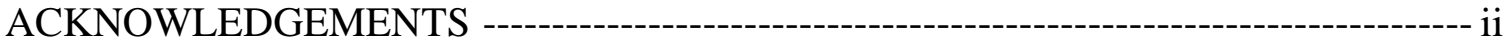

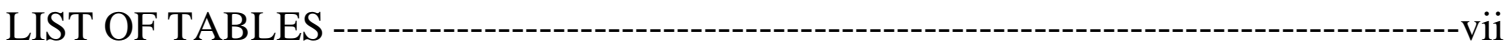

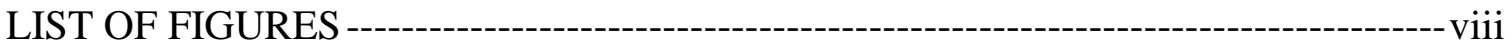

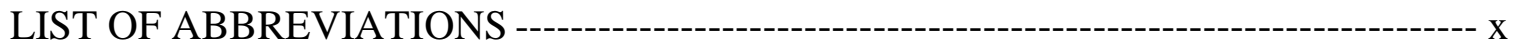

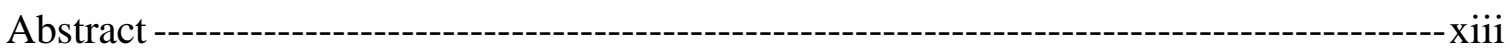

Chapter 1. Literature review ---------------------------------------------------------------------------- 1

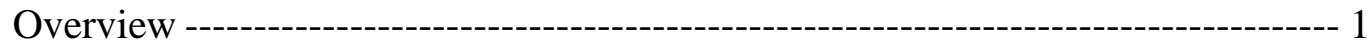

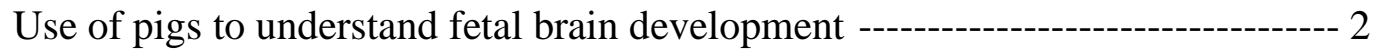

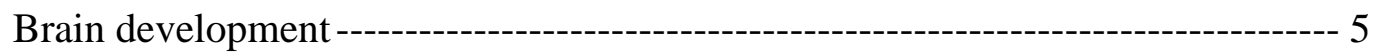

Epigenetics and fetal programing---------------------------------------------------------- 7

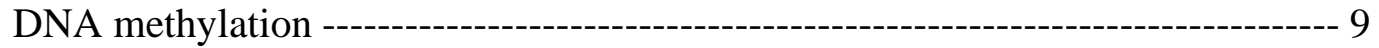

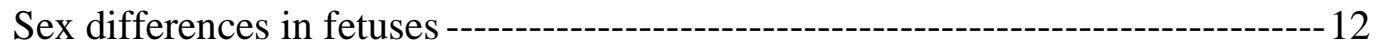

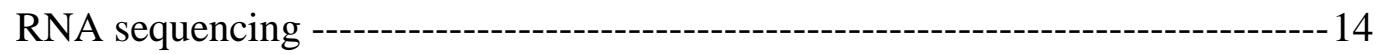

Chapter 2. Sexual dimorphic gene expression during fetal brain development in pigs --- 16

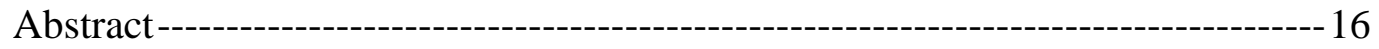

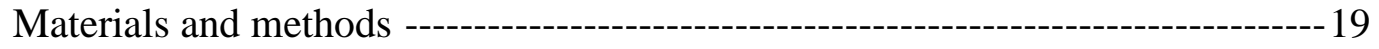

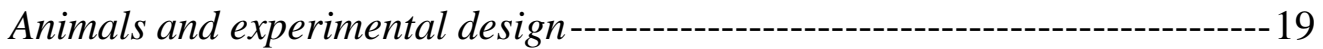

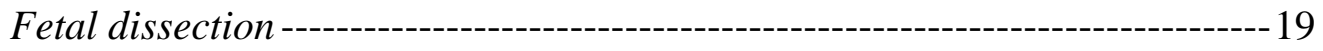

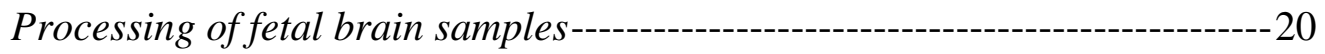


Gene expression profiling by RNA sequencing

RNA sequencing data analysis $-21$

Results 23

Developmental changes in the fetal brain weight

Developmental changes in gene expression of the fetal brain

Differential gene expression between the male and female fetal brain 25

Effect of the sex of adjacent fetuses on gene expression of the developing

brain $-26$

Bias in exon-level expression of the fetal brain

Integrative functional annotation of differentially expressed genes $-28$

Comparison of gene expression in the fetal brain and placenta 30

Discussion $-31$

Conclusion 34

Chapter 3. Epigenetic changes during fetal brain development in pigs ---------------------46

Abstract

Introduction

Materials and methods $-50$

Animals and experimental design $-50$

Fetal dissection $-50$ 
Blood collection

DNA extraction from blood 52

Enzymatic methyl sequencing $-52$

Analysis of the enzymatic methyl sequencing data 53

Results 55

DNA methylation of fetal brain at gestation day 45, 60 and 90 $-55$

Relationship between DNA methylation and gene expression in the fetal

brain $-57$

Methylation in the gene body and regulatory regions $-58$

Methylation of single nuclei polymorphic sites $-59$

Sex differences in methylation of the fetal brain-

Canonical correlation in DNA methylation between the fetal brain and adult

blood $-61$

Discussion

Conclusion $-67$

Chapter 4. Comparative analysis of gene expression between the fetal brain and placenta of pigs and mice 82

Abstract $-82$

Introduction 83 
Materials and methods

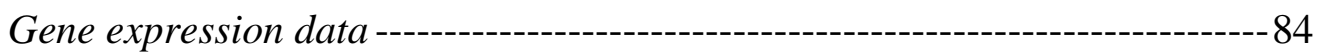

Identification of orthologous genes-----------------------------------------------84

Identification of genes expressed either in the fetal brain or placenta--------85

Prediction of transcription factors that control gene sets in pigs and mice -- 85

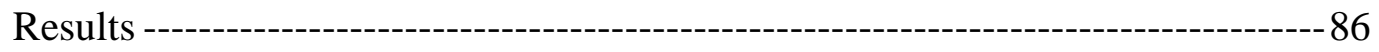

Identification of genes that show an 'ON/OFF' expression pattern in the

fetal brain or placenta of pigs ----------------------------------------------------86

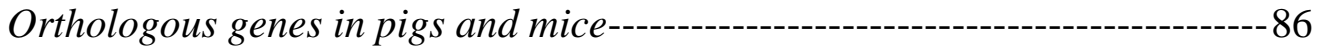

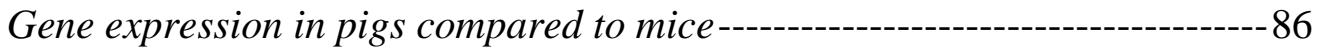

Prediction of transcription factors that target the common genes ------------- 87

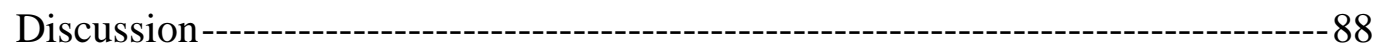

Conclusion ---------------------------------------------------------------------------------93

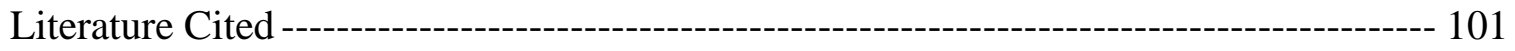




\section{LIST OF TABLES}

Table 2.1. The number of significantly differentially expressed exons (upregulated or downregulated) in the developing male and female fetal brain of pigs between gestation day 45,60 and 90 .

Table 2.2. The number of significantly differentially expressed exons (upregulated or downregulated) in the developing male and female fetal brain of pigs between gestation day 45,60 and 90 .

Table 2.3. PANTHER pathways that were significantly over-represented by the differentially expressed genes in the developing male and female fetal brain.

Table 3.1. The number and location of methylations in the gene body and the immediate flanking DNA of genes on gestation day 45, 60 and 90 in the fetal brain.

Table 3.2. The number of $\mathrm{CpG}$ methylated sites within exons and introns, splice sites and untranslated regions of genes at gestation day 45,60 and 90 in the fetal brain

Table 3.3. Significant enrichment of binding motifs of specific transcription factors in sequences encompassing the methylation sites canonically correlated between the fetal brain and adult blood.

Table 3.4. Age matched adult blood between sows and boars

Table 4.1. The number of genes expressed in the fetal brain or placenta of pigs at gestation day 60 and 90 .

Table 4.2. The number of pig genes, at gestation day 60 and 90, expressed in the mouse fetal brain or placenta.

Table 4.3. The number and type of homology genes between pigs and mice 96 


\section{LIST OF FIGURES}

Figure 2.1. The weight of male and female pig fetuses $(\mathbf{A})$, the fetal brains $(\mathbf{B})$, the relative weigh of the fetal brains (brain weight per $100 \mathrm{~g}$ of fetal weight) $(\mathbf{C})$, and the length of the fetal brains (D) on gestation day 45, 60 and $90 .$.

Figure 2.2. Principal component analysis (A) and Hierarchical clustering (B) of gene expression variation of the male and female fetal brain on gestation day 45, 60 and 90. 39

Figure 2.3. A. A chord diagram showing the mutation information pattern of gene expression relationship between the fetal brain of males and females on gestation day 45 , 60 and 90. B. A tanglegram comparing clusters of the top 20 expressed genes in the pig fetal brain based on their expression patterns in males and females during development.

Figure 2.4. Volcano plots showing differential expression of genes in the female and male fetal brain between gestation day 45, 60 and 90 .

Figure 2.5. Venn diagrams showing the number of upregulated and downregulated genes in the female and male fetal brains at gestation day 45, 60 and 90. 42

Figure 2.6. Network plots of genes based on mutation information of expression changes during development of the female fetal brain (A) and the male fetal brain (B). 43

Figure 2.7. A. A donut plot showing the proportion of marker genes representing different brain cells. B. A heatmap showing the number markers genes associated with different cell types in the brain and the developmental time periods during which those genes were significantly differentially expressed in the male and female fetal brain at gestation day 45,60 and 90 .

Figure 2.8. Violin plots of expression changes in marker genes specific to neurons (A) and astrocytes (B) of the male and female brain at gestation day 45, 60 and 90.

Figure 3.1. Venn diagrams showing the number of methylation sites between gestation day 45, 60 and 90 (A and B). Volcano plots showing hypomethylated and hypermethylated sites in the fetal brain on gestation day 45, 60 and 90 (C and D). 72

Figure 3.2. Chromosome-wise density plots of $\mathrm{CpG}$ methylation sites in the fetal brain that were common between gestation day 45, 60 and 90 (A and B). Common methylation across gestation day 45, 60 and $90(\mathbf{C})$.

Figure 3.3. A. Venn diagram showing the number of sex-specific methylation sites in the fetal brain. B. A circular Manhattan plot of the $-\log 10$ FDR values of differentially methylated $\mathrm{CpGs}$ between the male and female fetal brain in each chromosome. $\mathbf{C}$. Methylation density of the female fetal brain in each chromosome. D. Methylation density of the male fetal brain in each chromosome. 
Figure 3.4. Hierarchical cluster analysis of DNA methylation (A) and gene expression (B) among the male and female fetal brain samples on gestation day 45, 60 and 90. Pairwise correlation in methylation $(\mathbf{C})$ and gene expression $(\mathbf{D})$ among the male and female fetal brain on gestation day 45, 60 and 90. E. A cartoon depicting the inverse relationship in variation of DNA methylation with gene expression during development of the fetal brain from gestation day 45 to 90 . F. Image and weight of the fetal pig brain on gestation day 45 and 90 .... 75

Figure 3.5. A boxplot showing four groups of $\mathrm{CpG}$ sites that were methylated in a canonical correlated manner between the fetal brain and adult blood of pigs.

Figure 3.6. An arch plot showing the pair-wise mutual information network among methylation sites to which APOE (Apolipoprotein E) is the closet gene.

Figure 3.7. Mutual information network plots of the methylation levels among sites in chromosome $\mathrm{X}$ in males (A) and females (B) across the fetal brain and adult blood of pigs.

Figure 3.8. A. Venn diagram showing the number of sex-specific methylation sites in blood DNA of adult females and males. B. A circular Manhattan plot of the $-\log 10 \mathrm{FDR}$ values of differentially methylated $\mathrm{CpGs}$ between the male and female adult blood in each chromosome. C. Methylation density of the female adult blood in each chromosome. D. Methylation density of the male adult blood in each chromosome....... 79

Figure 3.9. Principal component analysis of methylation sites in adult sows and boars and in the fetal brain at gestation day 45, 60 and 90 that were associated with clusters (A) and those not associated with clusters $(\mathbf{B})$

Figure 3.10. A plot showing the observed and predicted log fold change values of methylation sites showing canonical correlation in methylation between the fetal brain and adult blood

Figure 4.1. Venn diagrams comparing the pig genes expressed in the fetal brain but not expressed in the placenta (A) at gestation day 60 and 90 or expressed in the placenta but not expressed in the fetal brain at gestation day 60 and 90 (B).

Figure 4.2. Venn diagrams comparing the number of genes expressed in the pig and mouse fetal brain but not expressed in the placenta of either species (A) or expressed in the placenta but not expressed in the fetal brain $(\mathbf{B})$.

Figure 4.3. Venn diagrams comparing the 1:1 orthologous genes and the number of genes expressed in the pig fetal brain but not expressed in the placenta at gestation day 60 and 90 (A) and 1:1 orthologous genes compared to genes expressed in the placenta but not expressed in the fetal brain at gestation day 60 and 90 (B).

Figure 4.4. Description of one-to-one orthologous genes in pigs and mice. 100 


\section{LIST OF ABBREVIATIONS}

\begin{tabular}{|c|c|}
\hline $5 \mathrm{hmC}$ & 5-hydroxymethylcytosine \\
\hline $5 \mathrm{HT}$ & 5-hydroxytryptamine \\
\hline $5 \mathrm{mC}$ & 5-methylcytosine \\
\hline AI & Artificial insemination \\
\hline APOBEC & Apolipoprotein B mRNA editing enzyme, catalytic polypeptide \\
\hline AUC & Area under curve \\
\hline BECon & Blood-Brain Epigenetic Concordance \\
\hline bp & Base pair \\
\hline BPA & bisphenol A \\
\hline $\mathrm{C} / \mathrm{T}$ & Cytosine $(\mathrm{C})$ / thymine $(\mathrm{T})$ base pairs \\
\hline $\mathrm{CC}$ & Canonical correlation \\
\hline cDNA & Complementary DNA \\
\hline ChiP-seq & Chromatin immunoprecipitation sequencing \\
\hline Chr & Chromosome \\
\hline $\mathrm{cm}$ & Centimeter \\
\hline CNS & Central nervous system \\
\hline $\mathrm{CpG}$ & cytosine and guanine separated by only one phosphate group \\
\hline CpG-SNP(s) & SNPs that map to $\mathrm{CpG}$ methylation sites \\
\hline $\mathrm{d}$ & Day (of gestation) \\
\hline DEG(s) & Differentially expressed gene(s) \\
\hline DevAge & Developmental aging \\
\hline $\operatorname{DMS}(s)$ & Differential methylation site(s) \\
\hline
\end{tabular}




\begin{tabular}{|c|c|}
\hline DNA & Deoxyribonucleic acid \\
\hline DOHaD & Developmental Origins of Health and Diseases \\
\hline EDTA & Ethylenediaminetetraacetic acid \\
\hline EM-seq & Enzymatic methyl sequencing \\
\hline $\mathrm{F}$ & Female \\
\hline FDR & False discovery rate \\
\hline FPKM & Fragments per kilobase million \\
\hline g & $\mathrm{g}$ force \\
\hline g & Gram \\
\hline $\mathrm{G} / \mathrm{A}$ & Guanine $(\mathrm{G})$ / adenine $(\mathrm{A})$ base pairs \\
\hline GEO & Gene expression omnibus \\
\hline GnRH & Gonadotropin-releasing hormone \\
\hline GO & Gene Ontology \\
\hline HOMER & Hypergeometric Optimization of Motif EnRichment \\
\hline $\operatorname{logFC}$ & Log fold change \\
\hline M & Male \\
\hline MAPK & Mitogen-activated protein kinase \\
\hline MB & Megabase \\
\hline MI & Mutual information \\
\hline $\mathrm{ml}$ & Milliliter \\
\hline MRI & Magnetic resonance imaging \\
\hline MRMR & Maximal relevance minimum redundancy \\
\hline mRNA & Messenger RNA \\
\hline
\end{tabular}




$\begin{array}{ll}\text { M-value } & \text { log ratio of methylated to unmethylated read counts } \\ \text { NES } & \text { Normalized enrichment score } \\ \text { ng } & \text { Nanogram } \\ \text { PCA } & \text { Principal component analysis } \\ \text { polyA } & \text { Polyadenylate } \\ \text { REST } & \text { RE1-Silencing Transcription factor } \\ \text { RNA } & \text { Ribonucleic acid } \\ \text { RNA-seq } & \text { RNA sequencing } \\ \text { SNP(s) } & \text { Single nucleotide polymorphism(s) } \\ \text { TATA } & \begin{array}{l}\text { A DNA sequence that indicates where a genetic sequence can be read } \\ \text { and decoded }\end{array} \\ \text { TF } & \text { Transcription factor } \\ \text { UTR } & \text { Untranslated region } \\ & \text { X-chromosome inactivation } \\ & \text { Microliter }\end{array}$




\title{
Molecular Regulation of Fetal Brain Development in Pigs
}

\author{
Monica P. Strawn \\ Dr. Susanta K. Behura, Thesis Supervisor
}

\begin{abstract}
Two experiments were conducted to investigate molecular regulation that impacts fetal brain development in pigs. In the first experiment (Chapter 2), gene expression was profiled by RNA sequencing (RNA-seq) to examine the whole transcriptome of the male (M) and female (F) fetal brain at gestation day (d) 45, 60 and 90. The analysis showed fewer differentially expressed genes (DEGs) in the brain of male and female fetuses in earlier gestation (d45-d60) when compared to late gestation (d60-d90). The homeobox (HOX) A5 gene that regulates pattern formation in early development was in the top upregulated DEGs between d45 to d60 in fetuses of both sexes. This study also found HOX B5 and D3 genes were in the top upregulated genes between d45 and d60 of the fetal brain of females, but not males. The second experiment (Chapter 3) investigated DNA methylation in pigs. DNA methylation in the fetal brain of both sexes at the same three gestation days was performed by enzymatic methyl sequencing (EM-seq). Hotspots of methylation in specific chromosomal regions were observed in the analysis. The analysis identified 1,475 sites in the pig genome that were methylated in the fetal brain, irrespective of sex, during development. The same sites were methylated in a canonically correlated manner in the blood of the adult stage, both in sows and boars. This is consistent with the Dilman theory of developmental aging (DevAge), which suggests that aging and early development of the brain are regulated by common molecular processes. A comparative analysis (Chapter 4) compared the gene expression patterns in the fetal
\end{abstract}


brain and placenta between pigs and mice. The analysis identified 112 genes that were expressed (mean FPKM > 10) in the fetal brain of both species but not expressed (mean FPKM $<1$ ) in the placenta of either species, and 10 genes that were expressed in the placenta of both species but not expressed in the fetal brain. In-silico analysis of the transcription factor binding sites in the $500 \mathrm{bp}$ of the upstream DNA of these common genes revealed that they were commonly regulated by the RE1 silencing transcription factor (REST), which is a multifaceted transcription factor that acts as a master regulator of neurogenesis as well as controls neural excitation and the aging processes. 


\section{Chapter 1}

\section{Literature review}

\section{Overview}

Due to ethical challenges associated with research on the human fetus, development of suitable animal models is necessary to better understand the biology of fetal development. How a fetal brain develops represents a fundamental question in biology. Development of the central nervous system (CNS) is a highly coordinated spatiotemporal process that includes the proliferation of glia and neurons and their migration, followed by programmed cell death, formation of synapses, myelination, and establishment of neuronal circuits. There is a remarkable similarity among the placental animals in the process of cortical development during fetal brain formation (Niemann and Rath, 2001). In humans, placental dysfunction can lead to defective neuronal development that can lead to brain disorders such as autism spectrum or attention deficit hyperactivity disorders in early childhood or chronic neuropsychiatric diseases such as schizophrenia, depression, and dementia later in life (reviewed by Bronson and Bale, 2016). While research on fetal brain development is gaining momentum using mice models, no largeanimal model has been widely used.

Links between lifespan and reproduction have been hypothesized from studies that observed correlations between gestation times and maximum life span among different mammals, placental shape and lifespan of tall and short men, and lifespan and fertility of women (Barker et al., 2011; Müller et al., 2002). Though these studies have suggested that reproduction and lifespan may have common regulatory mechanisms, no study has 
directly demonstrated the functional relationship between the two. This is a major gap in knowledge to understanding the placental role in offspring health and aging of the brain. In addition, the aging brain is susceptible to chronic diseases, some that occur in a sexbiased manner. Though a male fetus is generally associated with a higher risk than a female fetus in utero, the early-life origin of sexual dimorphic aging remains a mystery.

The domestic pig originated from the Eurasian wild boar (Sus scrofa) (Giuffra et al., 2000), but since its domestication it has been used for considerably more than a traditional livestock animal. Pigs are frequently used in human pharmacology and as a biomedical model in research. Pigs are a litter bearing species with a short gestation cycle, an early age to maturity, are similar to humans anatomically and physiologically, and has a well-established reference genome, which all makes pigs an ideal research model for gene expression and methylation patterns of biological aging during development. Many impairments or dysfunctions during pregnancy, including but not limited to disease, toxins, stress, and poor nutrition can disrupt normal development, have long lasting consequences on brain development, biological aging, and epigenetic changes within an individual (O’Connor et al., 2003; Shiau et al., 2020; Vassoler et al., 2013). Although none of these are new fields of study, there is plenty of information that remains unanswered about how these complex processes work in both humans and animals.

\section{Use of pigs to understand fetal brain development}

Pigs have been domesticated for thousands of years and were one of the earliest research animals. They were used to understand the mechanics of breathing in ancient 
Greece (304-250 B.C.), and to study blood circulation in Rome (130-200 A.D.) (Bollen et al., 2010). Currently, interest in pigs spans a wider range than perhaps any other domestic animal since they are used for food, biomedical research and are also, increasingly, a pet as well (Laber et al., 2015). Reproduction and development in pigs have been extensively studied. As pigs are used for commercial meat production, breeders and researchers constantly search for superior reproductive and developmental traits in pigs (Knox, 2014; Niemann and Rath, 2001; Wells and Prather, 2017; Zak et al., 2017).

The reproductive cycle in pigs, including ovulation, the endocrine processes of follicular and luteal phases, as well as an imperative role of nutrition and metabolism on pregnancy, have all been widely studied (Soede et al., 2011). The gestation period generally can vary from 110 to 118 days but averages 115.5 days. A normal fertile production dam gives birth to 11-16 piglets (litter size) with birth weights ranging from 900 to 1600 grams. Dams of some strains, used mostly in research, have more variety in average gestation length, litter size, and birth weight, depending on the genetics of the strain. The suckling period of newborns is about a month with common weaning between 3 and 5 weeks of age, occasionally longer. Puberty begins from 20 to 28 weeks after birth and the natural average life expectancy varies from 14-18 years.

Fetal development and organogenesis research in pigs have increased substantially for two primary reasons. One of the interests is focused on the use of pigs as a food animal for increased growth and development (Hard and Anderson, 1979; Ji et al., 2017; Leenhouwers et al., 2002; McPherson et al., 2004) while the other interest is focused on the use of pig organs for transplantation needs in humans (Eventov-Friedman et al., 2005; Hammerman, 2014; Rothkötter et al., 2002). The majority of these studies have focused 
on observational evidence of different conditions or factors during gestation on fetal organs, so the cellular and molecular factors that regulate organogenesis and organ development in pig fetuses are poorly understood (Foxcroft et al., 2006; Michael et al., 1983; Oksbjerg et al., 2013; Renegar et al., 1982).

In biomedical research, when it is unethical to use human subjects, pigs have several advantages over other animal species because they have favorable reproductive capabilities, and much of their anatomy and physiology are analogous to humans (Laber et al., 2015). As a human model, pigs work well specifically for brain and CNS growth and development because they share several genomic, biochemical, physiological, and anatomical characteristics (Chen et al., 2019; Swindle et al., 2012). For example, the size of a pig brain is more comparable than a rodent brain to the size of a human brain, pigs have a gyrencephalic brain, meaning that the cerebral cortex has ridges and groves, in contrast to the smooth or lissencephalic brain pattern found in rodents, they also exhibit a similar white to grey matter ratio to humans and have similar developmental peaks (Swindle et al., 2012). Pig studies, in general, have also shown to be more predictive of therapeutic treatments in humans than rodent studies (Schachtschneider et al., 2015). Dickerson and Dobbing (1967) found that fetal pigs most rapid period of brain growth lasted from 50 days before birth to 40 days afterwards (Dobbing, 1990). These results were reiterated by Dobbing and Sands (1979) stating that pigs and humans have similar growth curves with the most rapid growth near birth, then tapering off postnatally with similar percentages of the adult brain weight at birth (27\% for humans, $25 \%$ for pigs). Humans and pigs also have similar blood flow characteristics to the brain (Swindle et al., 2012). Pigs are less expensive and ethically more acceptable than canines and non-human 
primates (Schachtschneider et al., 2015). All these combined anatomical and physiological similarities to humans make pigs an ideal research model for gene expression in the developing brain as well as methylation pattern changes during biological aging.

In the genome, large methyl groups, added during DNA methylation, project into space and act to repress transcription (Hotchkiss, 1948). These changes to DNA transcription throughout life impact the biological age of individuals and can be tracked during research trials. While mice, as a model, are easier to house for research purposes, many human neurological changes are inadequately modeled in rodents (Chen et al., 2019), possibly due to the longer telomere length leading to a vastly different maintenance system and mice not reaching critical lengths during their short life span (Jylhävä et al., 2017). Inadequate neurological changes modeled in rodents have led to suggestions that pigs may be a more ideal animal model to understand aging of the human brain at the transcriptional level (Chen et al., 2019).

\section{Brain development}

Proper development of the fetal brain is crucial for successful life postnatally, but development and gene expression can be disrupted by many genetic and environmental factors such as disease, toxins, stress, poor nutrition, and genetic mutations (O'Connor et al., 2003; Shiau et al., 2020; Vassoler et al., 2013).

Development starts with thickening of the cranial surface neuroectoderm and formation of the neural plate which is bound laterally by the neural folds (Dyce et al., 2009; Noden and De Lahunta, 1985). As development continues, the folds become 
increasingly prominent and bend inward toward each other, eventually meeting and fusing to form the neural tube (Dyce et al., 2009; Noden and De Lahunta, 1985). Growth of the cephalic neural tube eventually forms the five distinct regions of the mature brain (Dyce et al., 2009; Noden and De Lahunta, 1985).

Dickerson and Dobbing (1967) found that the growth of the spinal cord in fetal pigs rose gradually during the last 50 days of gestation and was maintained for many months of postnatal life. The brain experiences a rapid period of growth from 50 days before birth to 40 days afterwards, during which it grew at a rate of 5-6\% of its adult weight biweekly (Dobbing, 1972). The cerebellum, which is located at the back of the brain and is attached to the brain stem, has a much more rapid rate of increase in weight and the period of growth is much shorter. It accounts for $4 \%$ of the weight of the brain at gestation day 80 and reaching its mature value of $10-11 \%$ at birth (Dickerson and Dobbing, 1967). In pigs, neurogenic cells in the hindbrain stop dividing earlier in gestation than the forebrain, leading to more advanced development of the cerebellum in pigs and other animals that walk within hours after birth (Noden and De Lahunta, 1985). The cerebellum only weighs $10-11 \%$ as much as the cerebral cortex, but its surface area is about $75 \%$ of that of the cerebral cortex (Barrett et al., 2010). Due to the cerebellum's involvement in maintenance of balance and posture, coordination of voluntary movements, and motor learning (Barrett et al., 2010; Dyce et al., 2009; Jennings, 2004), it is not surprising that it is highly developed in pigs at birth. Motor commands are not initiated in the cerebellum, but it ensures that movements are executed as intended by modifying the motor commands of the descending neural pathways and making the movements smooth and accurate (Dyce et al., 2009; Jennings, 2004). 


\section{Epigenetics and fetal programing}

Studies have shown that epigenetic and metabolic changes at early developmental stages can impact offspring health (Pinney and Simmons, 2012; Ruchat et al., 2013), including metabolic disorders of the brain (Kanekar and Gustas, 2011; Yoon et al., 2014). Epigenetics and metabolomics can be functionally linked to each other and epigenetic modification in the fetus can cause metabolic disorders in the adult (Bouchard, 2013; Christoforou and Sferruzzi-Perri, 2020; Sookoian et al., 2013; Tzika et al., 2018). Methylation accumulates at specific sites in the genome in a correlated manner with age, and those sites are commonly referred to as the 'epigenetic clock' (Horvath, 2013; Stubbs et al., 2017). Age-related methylation occurs differentially in different organs, so the epigenetic clock can be used to predict biological age of different organs, including the brain (Stubbs et al., 2017). Studies have also suggested that aging and longevity processes are linked to early-life events including reproductive conditions (Aguilaniu, 2015; Bianco-Miotto et al., 2017). Understanding how early life developmental processes influence health and well-being later in life is an important research area that falls under the broad umbrella of research efforts collectively termed as the Developmental Origins of Health and Diseases (DOHaD) (Barker, 2007; Wadhwa et al., 2009). The foundation of these research efforts is based on the Barker hypothesis (Barker and Osmond, 1986), which suggests that adverse conditions during intrauterine life and early developmental stages can cause irreversible alteration in physiology and metabolism that lead to heightened risk of diseases in adulthood. These projects not only benefit the knowledge of the early-life origins of human diseases, but also bear significance to public health and society at large (Penkler et al., 2019). With the advent of DOHaD research coordination 
in the early 2000s, diverse research themes have emerged to address basic and translational aspects of early-life origins of human health and diseases (Gage et al., 2016; Heindel and Vandenberg, 2015). Research interest includes examining molecular mechanisms that commonly control fetal development and aging processes, and the Dilman theory of developmental aging (DevAge) (Dilman, 1971). Recent studies using genomics and systems-biology approaches have supported the DevAge theory and have further revealed that the developmental aging link is a highly conserved mechanism in animals (de Magalhães and Church, 2005; Feltes et al., 2015).

When determining aging of humans and animals, it is important to note differences in chronological and biological age. Chronological age is a typical measurement of how long ago a person or animal was born (Lowsky et al., 2014). Chronological age is a major risk factor for functional impairments, chronic diseases, and mortality, but it lacks clear reasons for major physiological deterioration in individuals within a species at various chronological ages (Lowsky et al., 2014). Estimating biological age uses epigenetic biological markers of aging of an individual's DNA methylation data to better estimate the health of the individual (Simpkin et al., 2016). This estimation works because methylation patterns of DNA change as animals age (Martin, 2005; Singhal et al., 1987).

When predicting biological age, research interest is in epigenetics which is defined literally as "in addition to genetics". However, it better refers to changes in DNA accessibility or the surrounding chromatin that influence gene expression but do not change the genetic composition (McCarthy et al., 2009). Virtually all cells in an organism contain the same genetic information, but not all genes are expressed the same way by all cell types because of epigenetic modifications in the genome. Therefore, epigenetic 
mechanisms help mediate the diversified gene expression profiles in a variety of cells and tissues. Prediction of biological age using epigenetic changes works because the critical regulatory portions of the genome remain under strict epigenetic control throughout life (Bocklandt et al., 2011; Martin, 2005). Biological age therefore is determined using biological parameters (Hannum et al., 2013) that are strongly correlated to age and better predict functional capability than chronological age (Jylhävä et al., 2017). Genetic variants cannot be used in place of epigenetic changes because the genome sequence is relatively static over time (Hannum et al., 2013). In previous studies it has been shown that genes with age-associated expression were more likely to have nearby age-associated methylation markers (Hannum et al., 2013) that can be used to predict biological age. Although it is known that epigenetic changes to DNA regulate genome expression, an epigenetic change does not always result in immediate alteration of gene transcription but may alter the response to future signaling events (Chung and Auger, 2013).

\section{DNA methylation}

The pig genome is comprised of 18 autosomes, with $\mathrm{X}$ and $\mathrm{Y}$ sex chromosomes and at $2.7 \mathrm{~Gb}$ it is similar in size to the human genome (Groenen et al., 2012). Gene expression can be controlled in many ways in eukaryotes, and one common epigenetic

tool used is DNA methylation. DNA methylation, along with histone modifications and microRNA, do not change the DNA sequence but alter the likelihood of a gene being transcribed and translated into a protein. During methylation, gene expression can be repressed by large methyl groups interfering with binding of transcription factors or by triggering chemical reactions that encourage the DNA to become tightly wrapped and 
difficult to access (Wu et al., 2010). This process has been of significant interest after first being discovered in mammals in the 1940s (Avery et al., 1944; McCarty and Avery, 1946). After discovery, many researchers proposed DNA methylation might regulate gene expression, but it was not until the 1980s that several studies demonstrated that DNA methylation was involved in gene regulation and cell differentiation (Compere and Palmiter, 1981; Holliday and Pugh, 1975). Since then, it has been linked to specific control of genes and has been shown to be linked to diseases in humans and animals.

DNA methylation is a process by which methyl groups are added to the DNA molecule, most commonly the covalent addition of a methyl group at the 5-carbon of the cytosine ring resulting in 5-methylcytosine (5mC) (Hotchkiss, 1948). The $5 \mathrm{mC}$ content of DNA from different tissues or cell types can vary considerably even when tissues from the same individual are compared (Ehrlich et al., 1982). In mammalian DNA, more than 98\% of DNA methylation occurs in a CpG dinucleotide context in somatic cells (Bernstein and Jin, 2015; Lister et al., 2009). The major exception is CpG islands that are often located near promoters as they remain unmethylated allowing gene expression to occur (Bernstein and Jin, 2015). There is also supporting evidence that similar methylation patterns are not seen in embryonic stem cells, suggesting that these stem cells may have a different mode of regulation (Lister et al., 2009). Overall, in the adult genome, these large methyl groups project into space and repress transcription (Hotchkiss, 1948). The level of DNA methylation is higher in the brain than other tissues, suggesting that the brain has very tight gene regulation via methylation patterns (Ehrlich et al., 1982; Tawa et al., 1990; Wilson et al., 1987). 
DNA methylation patterns change with increasing age and environmental stress (Hannum et al., 2013), contribute to age related diseases (Bocklandt et al., 2011; Singhal et al., 1987) and act as biomarkers of aging that are highly correlated with chronological age (Simpkin et al., 2016). There has been increased research interest in epigenetic changes and the ability to use these changes to aid in cell or tissue aging estimates. Although there are many epigenetic changes, DNA methylation has received the most attention in epigenetic aging research. Studies have shown that during the brain aging process in pigs, upregulated genes were mainly related to stress and immune response, and the downregulated genes were mainly involved in neuronal functions (Chen et al., 2019), similar results to humans and macaques (Erraji-Benchekroun et al., 2005; Lu et al., 2004; Somel et al., 2010).

A CpG site is a cytosine and guanine separated by only one phosphate group indicating that they are nucleotides right next to each other in the DNA sequence. Some CpG islands, a region of the DNA with a high frequency of $\mathrm{CpG}$ sites, are associated with a strong tendency to hypermethylate specific loci and have been described in colorectal cancers, gliomas and unexpected methylation patterns have been reported in breast cancer (Fang et al., 2011). Cancer-specific alterations in DNA methylation are hallmark measurements of malignancies (Fang et al., 2011). Methylation patterns not only play a role in cancer, but also in many other biological processes like memory formation and neurological disorders. There have been previous studies that have shown methylation plays a dynamic role in neurons by modulating key gene activities (Chen et al., 2003; Martinowich et al., 2003) and that memory function is, at least partially, driven by methylation patterns (Feng et al., 2010; Miller et al., 2008). 


\section{Sex differences in fetuses}

Sexual dimorphisms are defined as a phenotypic difference between males and females of a species and are a common phenomenon in animals (Parés-Casanova, 2013). For example, domestic pigs are generally male-larger but much less dimorphic than most of their wild relatives (Parés-Casanova, 2013). However, sex differences go much further than phenotypic differences in size and weight. The vertebrate brain is organized in a sexdependent fashion with differences at every level of brain organization, (Bakker and Baum, 2008; Savic et al., 2010) due to, at least in part, the control of perinatal gonadal steroid hormones (Savic et al., 2010). In mammals, the role of epigenetics in sex differences begins early in development (McCarthy et al., 2009), leading to extensive and diverse differences in growth and organization.

Many mammalian species show differences in brain development between males and females (Cooke et al., 1999). Sexual dimorphism in the structure, development and function is well documented in the human brain (McCarthy et al., 2012; Pallayova et al., 2019). In addition, sex differences in placenta development and function have also been noted (Rosenfeld, 2015). Previous studies have shown, primarily in rodents, that the placenta developmental rate is faster in male fetuses than females (Kalisch-Smith et al., 2017). In humans, specific age-related brain diseases occur in disproportional frequency in one sex over the other, which is hypothesized to have links with the sexual differences in the brain at the fetal stage (McCarthy et al., 2012). A male fetus is generally associated with a higher risk of death than a female fetus (Kraemer, 2000). Many problems during gestation, including brain disorder, cerebral palsy, and premature and still birth occur at higher rates in males than females (Kraemer, 2000; McCarthy et al., 2009). Although 
human males generally weigh more than females at birth, physiologically they are 4-6 weeks behind females (Gualtieri and Hicks, 1985).

In humans, male brains have consistently been reported to be $8-10 \%$ larger than female brains (Wierenga et al., 2019), and Tekola-Ayele et al. (2019) stated that in males they also observed a larger head circumference and longer lengths at birth than in females. Research also shows that male fetuses have higher in-utero energy demand than female fetuses, playing a role in early epigenetics as well as biological aging through their lifetime (Tekola-Ayele et al., 2019).

DNA methylation patterns have been found to accurately estimate age using blood (Hannum et al., 2013; Horvath et al., 2016; Simpkin et al., 2016), but Hannum et al. (2013) found further correlations in many tissues of the human body. They found that in each tissue studied, including the brain, males appeared to age more quickly than females, providing evidence that these trends are not specific to blood (Hannum et al., 2013). Horvath et al. (2016) found similar results that males exhibited a higher age acceleration, specifically in the cerebrum of the brain, than those from females. These analyses provide evidence that methylomic changes are intrinsic and not due to the changing cell-type composition of whole blood with age but instead are body wide changes (Hannum et al., 2013). This study, along with others, demonstrate that epigenetic aging rates differ between sexes and suggest more rapid immunosenescence in men than women (Horvath et al., 2016). Although a good deal is known about methylation patterns in humans, the relationship to morbidity and mortality in humans and animals is not fully understand. 


\section{RNA sequencing}

RNA sequencing (RNA-seq), used to measure the quantity of RNA in a biological sample at a given moment, was first developed in the mid 2000s and was used with nextgeneration sequencing technology by different groups (Bainbridge et al., 2006; Cheung et al., 2006; Emrich et al., 2007; Weber et al., 2007). In the last decade, it has revolutionized techniques to measure RNA by providing high throughput, quantitative analysis which is becoming even more common and affordable for research and medical purposes. RNAseq uses complementary DNA (cDNA) fragments which are subjected to high throughput sequencing and mapped to the species genome to measure the quantity of RNA from known and previously unknown genes (Nagalakshmi et al., 2008; Wu et al., 2010). RNAseq technology and techniques have flourished since their launch, growing in popularity, acceptance and uses for human and animal research and medicine. This technology gives researchers the ability to examine gene expression and gene regulation at the time of sample collection.

\section{Summary}

Pig genetics, anatomy, and physiology, as well as their reproductive cycles, make them a good candidate for a research model. Pigs exhibit brain development that is useful to profile gene expression and identify the epigenetic clock. Genetically manipulated pig models, based on rational designs in a highly specific manner (such as specific genes, regulatory factors, epigenetic sites etc.) with data from functional genomics studies, can also be used to promote research on fetal brain development. Functional genomic research includes gene regulation in male versus female developing fetal brains and genes 
that pinpoint specific DNA sites that are methylated in the promoter regions of those genes in a coordinated manner between the fetal stage and old age. 


\title{
Chapter 2
}

\section{Sexual dimorphic gene expression during fetal brain development in pigs}

\begin{abstract}
In the current study, the fetal weight was measured, as well as the length and weight of the developing brains, and the gene expression level of male $(\mathrm{M})$ and female $(\mathrm{F})$ fetuses at gestation day (d) 45, 60 and 90 was examined. The brain to fetal weight ratio (brain weight per $100 \mathrm{~g}$ of fetal weight) was lower at $\mathrm{d} 45$ compared to $\mathrm{d} 60$ and $\mathrm{d} 90$. The length of the fetal brain nearly doubled on d90 compared to d45. Gene expression was profiled by RNA sequencing (RNA-seq) to examine the whole transcriptome of the fetal brain at three gestation days. Daily fetal weight gain increased by nearly 3 times from d60 to $\mathrm{d} 90$ compared to $\mathrm{d} 45$ to $\mathrm{d} 60$, but there were no differences in body weight or brain weight of male compared to female fetuses. Fewer differentially expressed genes (DEGs) in the brain of male and female fetuses were identified in earlier gestation (d45-d60) when compared to late gestation (d60-d90). Overall, there was approximately a 3.5-fold increase in the number of genes that were altered between d60 and d90 compared to d45 and d60. A greater number of DEGs in late gestation is suggestive of preparation of the brain for postnatal function. The homeobox $(H O X) A 5$ gene, that regulates pattern formation in early development, was in the top upregulated DEGs between d45 and d60 in fetuses of both sexes. This study also found $H O X B 5$ and $D 3$ genes were in the top upregulated genes between d45 and d60 of the fetal brain of females, but not males. Myelin associated glycoprotein was among the most downregulated DEGs in both male and female fetuses during d60 to 90, and the gene coding for ERMN was one of the top
\end{abstract}


downregulated DEGs between d60 and d90 in both male and female fetuses. Male fetuses that were flanked by two female fetuses or by a male fetus on the medial side and a female fetus on the lateral side were identified. Gene expression in the developing brain of those males showed significant changes in expression. This analysis identified 54 DEGs between these 2 groups, suggesting that gene expression in the fetal brain is impacted by the sex of the adjacent fetuses. A comparative analysis of gene expression data of $\mathrm{d} 60$ and $\mathrm{d} 90$ identified 45 ion transporter genes that were coordinately regulated, in the form of ON/OFF expression, between the fetal brain and placenta of pigs. Analysis of the effect of intrauterine fetal location on the brain suggested ion transporters may be involved in a sex-biased regulation of the brain-placental axis.

\section{Introduction}

How a brain develops in the fetal stage represents a fundamental question in biology. Development of the central nervous system (CNS) is a complex and highly coordinated spatiotemporal process that includes the proliferation and migration of glia cells and neurons, followed by programmed cell death, formation of synapses, myelination, and establishment of neuronal circuits. There is a remarkable similarity among the placental animals in the process of cortical development during fetal brain formation (Dobbing, 1990). In humans, placental dysfunction is known to lead to defective neuronal development that can predispose to brain disorders such as autism spectrum or attention deficit hyperactivity disorders in early childhood or chronic neuropsychiatric diseases such as schizophrenia, depression, and dementia later in life (reviewed by Bronson and Bale, 2016; Shallie and Naicker, 2019). Previous studies have suggested that adaptive functions of the maternal placenta safeguard the fetus, specifically the brain, from injury 
and adverse conditions (Lye et al., 2015; Noden, and De Lahunta, 1985). While research on fetal brain development using mouse models has provided insights into factors that influence brain development (Semple et al., 2013), no large-animal model is being widely used. Due to ethical challenges associated with research on human fetuses, development of suitable animal models is necessary to better understand the biology of fetal brain development. This study focuses on the pig as an animal model to study brain development in male and female fetuses at gestation day 45, 60 and 90.

The study of gene expression profiling in the developing brain is important for a more comprehensive understanding of fetal development. RNA sequencing (RNA-seq) is currently a widely used technique to quantify gene expression in a genome-wide manner and identify the presence and prevalence of transcripts and splice forms from known and even previously unknown genes (Nagalakshmi et al., 2008; Wu et al., 2010). In mammals, regulation of gene expression plays an important role in sex differences of growth and development (McCarthy et al., 2009). Sexual dimorphisms are commonly acknowledged and studied in multiple mammalian species. Humans have been reported to have sexual differences in brain size, head circumference, energy demands, and biological aging postnatally (Hannum et al., 2013; Horvath et al., 2016; Tekola-Ayele et al., 2019; Wierenga et al., 2019). Differences in the vertebrate brain are associated with the early organization in a sex-dependent fashion with early control of perinatal gonadal steroid hormones that cause differences at every level of brain organization (Bakker and Baum, 2008; Savic et al., 2010). Previous research shows differences in gene expression between fetal sexes, as well as during periods of rapid growth and differentiation during development. In pigs, the fetal brain develops in an accelerated manner 50 days before 
birth to 40 days after birth (Dickerson and Dobbing, 1967) during which the brain is changing rapidly and preparing for postnatal life.

Knowledge about gene expression during brain development of male and female fetal pigs is limited. The long-term goal of this research project is to develop pig models that can be used to promote research on fetal brain development, disorder, and dimorphism. Towards that broad objective, the immediate aims were to study the fetal brain from male and female pigs at different gestation times to profile gene expression to test the hypotheses that 1) genes were coordinately regulated during brain development, and that 2) fetal sex, position in the uterus and the other fetuses influence gene expression in the developing brain.

\section{Materials and methods}

Animals and experimental design

In this study all 18 fetal pigs were obtained from the University of Missouri Swine Research Complex (Columbia, MO). Dams (n=9) were bred via artificial insemination (AI) and the day of AI was considered gestation day 1 (d1). Dams were Landrace, Large White or a cross of these breeds and bred to LR-M6 from Choice Genetics (Choice USA, 1415 28th Street Suite 400 West Des Moines, Iowa 50266). Dams were euthanized at d45 (44-46), d60 (59-61) or d90 (89-91) via electrical stunning and exsanguination at the University of Missouri abattoir, a USDA inspected commercial unit (Establishment \#5077A).

\section{Fetal dissection}


The reproductive tract was removed from the dam at the abattoir. Individual fetuses, referred to as $\mathrm{d} 45$, d60, or $\mathrm{d} 90$ male $(\mathrm{M})$ or female $(\mathrm{F})$ were selected based on the sex and removed from each uterine horn. One male and one female fetus was collected from each dam. No fetuses were taken from the tip of either horn or the body of the uterus. Each fetus was weighed before dissection. Additional information was taken from the litter including the total number of fetuses, and the sex of fetuses on the medial and lateral side of the fetus. The skin and skull were cut down the mid-sagittal plane with a scalpel and removed near the ears. The whole brain was scooped out, weighed, measured at the longest point, and stored on ice until homogenization.

\section{Processing of fetal brain samples}

Each fetal brain was homogenized with $5 \mathrm{ml}$ (d45) or 20ml (d60 and d90) RLT buffer (Qiagen, Cat No./ID: 79216) and 200 $\mu 1$ 2-mercaptoethanol. Homogenization was done in a 50ml Falcon tube using a benchtop VDI 25 tissue homogenizer (VWR). Aliquots of $1 \mathrm{ml}$ of the homogenate were diluted with 9-10ml RLT buffer and 10 $\mu 1$-mercaptoethanol to form a lysate. This was transferred into 1.5ml RNase/DNase-free Axygen conical tubes (Corning Axygen ${ }^{\circledR}$ MCT-175-C MaxyClear ${ }^{\mathrm{TM}}$ Snaplock Microcentrifuge Tube), frozen and stored at $-80^{\circ} \mathrm{C}$ for future analysis.

\section{RNA extraction}

Total RNA was isolated from frozen tissue samples using an AllPrep DNA/RNA Mini Kit (Qiagen, Cat No./ID: 80204) as per the manufacturer's instruction. The lysate was thawed then centrifuged for 1 minute at $\geq 8000 \mathrm{x}$ g. From the supernate, $750 \mu \mathrm{l}$ was 
transferred into RNase/DNase-free Axygen conical tubes (Corning Axygen, 1.7ml) and mixed with $350 \mu 170 \%$ ethanol to precipitate RNA. A DNase treatment step was added to remove genomic DNA contaminants. RNA was eluted in $30 \mu 1$ nuclease-free water twice for a total volume of $60 \mu \mathrm{l}$. RNA from a total of 18 samples (3 gestation days, 3 biological replicates, and 2 fetal sexes) was generated. Concentration and purity of the RNA was determined using Nanodrop 1000 spectrophotometer (Thermo Fisher Scientific) before each sample was diluted to $100 \mathrm{ng} / \mu \mathrm{l}$ using nuclease-free water.

\section{Gene expression profiling by RNA sequencing}

Illumina sequencing libraries were generated from the total RNA of each sample. Preparation of libraries and RNA sequencing (RNA-seq) were performed by the Novogen Coperation Inc. (8801 Folsom Blvd \#290, Sacramento, CA 95826). Each library was sequenced to 20 million paired end reads of 150 bases using a NovaSeq sequencer.

\section{RNA sequencing data analysis}

The quality of raw RNA sequence reads was checked with FastQC before trimming of adapters from the sequence reads using cutadapt. The quality reads were then mapped to the swine reference genome Sscrofa11.1 using Hisat2. The FeatureCounts program was used to quantify read counts that mapped to genes. The raw and processed data of RNA-seq are publicly available in the GEO database (accession GSE178970). The read count data were used in edgeR to identify the number of significantly expressed genes as described earlier (Behura et al., 2019a; Behura et al., 2020). In addition, the read count data were used to perform principal component, network and correlation analysis using 
R. Functional annotation of differentially expressed gene sets was performed by Gene Ontology (GO) and pathway analysis. GO and pathway enrichment analysis was performed by Fisher's exact test followed by multiple correction of raw p-values of significance using the analysis tool and up-to-date GO and pathway annotation databases of the PANTHER Classification System (Mi et al., 2013). The marker genes predicted from mammalian brain single-cell RNA-seq projects, available in the PanglaoDB (Franzén et al., 2019) were used in the integrative annotation of function of DEGs. The marker genes were downloaded from the freely available database based on the UCSC genome server (Franzén et al., 2019) and compared with the DEGs predicted from the bulk RNA-seq data of the pig fetal brain from the current study. A finite normal mixture model1 implemented in the $\mathrm{R}$ package mclust5 was used to predict gene expression clusters from the RNA-seq data. To infer gene expression networks within a predicted cluster, an information theory approach (Steuer et al., 2002) was used. In this method, mutual information (MI) of variation between gene expression was determined. MI measures the information content that two variables share: a numerical value ranging from 0 to 1 depending on, intuitively, how much knowing one variable would predict variability of the other. MI was calculated in a pair-wise manner between each pair of genes (read count data from RNA-seq) to generate a weighted adjacency matrix by the Maximum Relevance Minimum Redundancy (MRMR) method (Radovic et al., 2017). Mutual information network analysis was performed using the R package minet (Meyer et al., 2008). The comparative analysis of the hierarchical clustering (tanglegram analysis) of gene expression data was performed using the R package dendextend (Galili, 
2015). All statistical analyses, including the principal component analysis, and graphics were performed in $\mathrm{R}$.

\section{Results}

Developmental changes in the fetal brain weight

The mean fetal weight increased from $23.6 \mathrm{~g}$ on $\mathrm{d} 45$ to $113.2 \mathrm{~g}$ on $\mathrm{d} 60$. On d90, the mean fetal weight increased to $613.4 \mathrm{~g}$ (Figure 2.1A), indicating that the fetuses gained an average of $6.0 \mathrm{~g}$ per day between $\mathrm{d} 45$ and $\mathrm{d} 60$, and $16.6 \mathrm{~g}$ per day between $\mathrm{d} 60$ and d90. This is nearly a 3-fold increase in the daily weight gain between $\mathrm{d} 60$ and 90 as compared to between $\mathrm{d} 45$ and $\mathrm{d} 60$. On $\mathrm{d} 45$, there was a slightly higher weight of males compared to females (21.1g in females versus $25.9 \mathrm{~g}$ in males), but on $\mathrm{d} 60$ and $\mathrm{d} 90$, the mean weight of males and females was identical. The weight of the fetal brain was expressed as percentage values of the fetal weight (brain weight per 100g of fetal weight) for comparison among d45, 60 and 90. The weight ratio of brain-to-fetus was 5.7 on d45 whereas those on day d60 and d90 decreased to 2.6 and 3.1, respectively (Figure 2.1C). The weight ratio of brain-to-fetus on $\mathrm{d} 45$ tended to be slightly higher in female than male fetuses (6.3 in females compared to 5.1 in males; Figure 2.1C). However, the ratio between brain and fetal weight became identical in both sexes on d60 and d90. The length of the fetal brain more than doubled between $\mathrm{d} 45$ and d90 (Figure 2.1D). On d45, the mean length was $1.7 \mathrm{~cm}$. It increased to $2.1 \mathrm{~cm}$ by $\mathrm{d} 60$ and reached $3.9 \mathrm{~cm}$ on $\mathrm{d} 90$. The brain length was not different in males and females at any time points (Figure 2.1D).

Developmental changes in gene expression of the fetal brain 
Gene expression was profiled by RNA-seq to examine the whole transcriptome of the fetal brain on d45, 60 and 90. Principal component analysis (PCA) and hierarchical clustering demonstrated that at $\mathrm{d} 90$ the fetal brain is transcriptionally distinct from $\mathrm{d} 45$ and d60 (Figure 2.2A and 2.2B). Nearly 57\% of the variation in gene expression was explained by the first principal component. The observed variation was not surprising, as rapid growth of the fetal brain was observed between d60 and d90 (Figure 2.1B), indicating that changes in gene expression on $\mathrm{d} 90$ are likely associated with the rapid development of the fetal brain during this time. However, developmental changes in gene expression of the fetal brain are mutually informative between sexes and gestation time points (Figure 2.3A). Mutually informative variables in probability theory share common information called mutual information (MI), so one can know the dependence between the two variables (Steuer et al., 2002). This approach was used to quantify MI between fetal brains of two sexes and the three gestation time points in a pair-wise manner which showed the inter-dependence patterns (shown as arches connecting one sample to another) in Figure 2.3A. The pattern of connectedness of the arches connecting one sample to another indicates how gene expression changes in the fetal brain at $\mathrm{d} 90$ is dependent on the gene expression patterns earlier in development, on day 45 and 60. Besides examining global pattern analysis, expression changes at the individual gene levels were also investigated. The top 20 highly expressed genes were compared between the male and female fetal brain to examine cluster patterns in the expression level. The pair-wise Euclidean distances between gene expression variation were used for inferring hierarchical cluster patterns among these genes for comparison between the male and female brain (Figure 2.3B). The comparison of the cluster patterns shows three sub- 
clusters, shown by black, green and red lines connecting the nodes in the trees, where the male and female brain show variation in expression at individual genes. For example, CALMI and KIFIA were expressed in a tightly correlated manner in the male brain (they branch out from a common node), whereas their expression is more divergent in female brain (they have different common nodes).

Differential gene expression between the male and female fetal brain

Differential expression analysis by edgeR identified genes with significant changes in expression in the fetal brain during development between $\mathrm{d} 45$ and $\mathrm{d} 60$, and between $\mathrm{d} 60$ and d90. Figure 2.4 shows the wide-spread differential gene expression in the fetal brain of males and females during those developmental transitions. A greater number of genes were differentially expressed during development from $\mathrm{d} 60$ to $\mathrm{d} 90$ than that from $\mathrm{d} 45$ to d60. However, this pattern was reliant on the sex of the fetuses (F.d60 to F.d90 DEGs $=$ 5325; M.d60 to M.d90 DEGs= 6605; F.d45 to F.d60 DEGs = 2059; M.d45 to M.d60 DEGs=1323). The data showed that brain development from d60 to d90 in males is associated with greater number of DEGs, both upregulated and downregulated, compared to females (Table 2.1). The number of upregulated and downregulated genes between d45 and d60 and between d60 and d90 in males and females is shown in Figure 2.5. The male fetal brain showed approximately a 5 -fold increase in the number of DEGs between d60 and d90 compared to that from $\mathrm{d} 45$ and d60, but the female fetal brain only showed a 2.5-fold increase in the number of DEGs at the same time points. Considering that adaptive changes in gene expression are associated with the cost of cellular energetics (Wagner, 2005), these findings indicate that brain development in males is transcriptionally more energy expensive than brain development in females. In humans, it 
is known that gestation of male fetuses is often associated with a higher risk of intrauterine growth restriction and even death compared to of female fetuses (Alur, 2019; Kraemer, 2000; McCarthy, 2016).

\section{Effect of the sex of adjacent fetuses on gene expression of the developing brain}

While recording the sex of the fetuses analyzed in the current study, the sex of adjacent fetuses on the medial side (closer to the body of the uterus) and on the lateral side of the uterus (closer to the tip of the uterus horn) were also recorded to evaluate whether the sex of adjacent fetuses influenced the brain transcriptome. The representation of fetal sexes as M.F.M means that there was a male fetus collected where there was a female fetus on the medial side and a male fetus on the lateral side. The fetal collection included male piglets that were flanked either by two females (M.F.F) $(n=3)$ or by a male on the medial and a female on the lateral side (M.M.F) $(n=2)$. The differential gene expression analysis showed a significant impact on gene expression in the brain between the two groups. A total of 54 genes were impacted in the fetal brain of the males when they were flanked by fetuses of both sexes as opposed to fetuses of the same sex (in this case female). Of these genes, 34 genes showed significantly higher expression in the brain of male fetuses when there was a female fetus on the medial side in comparison to the fetal brain of the same sex (male). In addition, 20 genes showed significantly lower expression in the male fetal brains when there was a female fetus on the medial side. Of the 54 DEGs in the male brain due to sex differences of adjacent fetuses, 37 were annotated to encode known proteins and others were predicted coding genes. Interestingly, 16 of these 37 known genes were related to ion transport, a key component of normal feto-placental circulation (Jansson et al., 2009; Sangkhae and Nemeth, 2019). 
This study did not include age matched female fetuses that varied in the sex on the medial side, therefore those analyses were not included. Those groups will be compared in a future study.

\section{Bias in exon-level expression of the fetal brain}

This study quantified exon-level expression using featureCounts (Liao et al., 2014) to count the number of mapped reads to individual exons of each gene. Analysis of the exon-level expression in edgeR identified a total of 2,084 genes in which one or more exons were significantly differentially expressed during fetal brain development. A total of 2,774 differentially expressed exons were associated with these genes. As the brain develops from d60 to d90, a greater number of exons were differentially expressed. However, at the gene-level, a nearly equal number of upregulated and downregulated genes from d60 to $\mathrm{d} 90$ were observed. There was a bias in the number of exons that were less expressed on $\mathrm{d} 90$ compared to $\mathrm{d} 60$ in the female brain. On the other hand, there was a bias in the number exons with increased expression on $\mathrm{d} 90$ compared to d60 in the male brain (Table 2.2). The sex bias observed in this study suggest that gene expression of the fetal brain is significantly biased at the exon-level between male and female fetuses. The data further showed that there is a preferential bias in the expression of specific exons. The second and third exons were more likely to be differentially expressed than any other exons in the genes expressed in the fetal brain, irrespective of sex. Exon-rich genes showed an inverse relationship between expression and the exon rank (position in the gene from gene start to end codon). The higher ranked exons were less likely to be

differentially expressed in the fetal brain, suggesting that the number of exons of brain expressed genes is an intrinsic modular of gene regulation in the developing brain. 


\section{Integrative functional annotation of differentially expressed genes}

Gene Ontology (GO) analysis was performed to predict functions of the genes differentially expressed in the male and female brain between gestation day 45, 60 and 90. Significant enrichment of 1,349 GO terms were identified, many related to brain development. Of these, $308 \mathrm{GO}$ terms were commonly enriched among genes differentially expressed in fetal brain of both sexes during development from d45 to d60 as well as from d60 to d90. Besides this common set of GO terms, an additional $240 \mathrm{GO}$ terms were enriched in the female fetal brain, but only 30 additional GO terms were enriched in the male fetal brain, indicating that the male brain during development lacks functional genes related to $210 \mathrm{GO}$ terms that play an important role during the development of the female fetal brain. In the top 5 significant female specific GO terms $(\mathrm{n}=210)$ were biological processes related to cerebral cortex regionalization, negative regulation of cardiac muscle adaptation and hypertrophy in response to stress, and the cellular response to cGMP. Among the top 10 significant GO terms from the fetal brain of both sexes from d45 to d60 were biological functions related to cerebral cortex regionalization, pre-replicative complex assembly involved in cell cycle DNA replication, pre-replicative complex assembly involved in nuclear cell cycle DNA replication, and double-strand break repair via break-induced replication. But, as the brain develops from d60 to d90, functions related to glutamate secretion, regulation of axon extension involved in axon guidance, and regulation of neuronal synaptic plasticity were common among the top $10 \mathrm{GO}$ terms enriched in the brain of both sexes.

In addition to GO analysis, PANTHER pathway analysis was used to identify specific pathway(s) that were over-represented by the DEGs (Table 2.3). This analysis showed 
that endothelin signaling, gonadotropin-releasing hormone receptor signaling, angiogenesis, and Wnt signaling were commonly associated with the fetal brain developmental processes in pigs. However, this study also identified that specific pathways were enriched among the DEGs in a sex-specific manner. For example, the ionotropic glutamate receptor pathway and p53 pathway were enriched in the female brain but not impacted in the male brain. On the other hand, different receptor mediated signaling pathways were enriched in the male brain alone (Table 2.3). Hierarchical cluster analysis identified genes that were expressed in a coordinated manner among each other (Figure 2.6). Mutual information network inference (Meyer et al., 2008) further supported the cluster analysis that specific DEGs are expressed in a mutually informative manner (Steuer et al., 2002) in the developing fetal brain. For example, the 25 genes shown in Figure 2.6 were identified not only as significant DEGs from edgeR analysis, but they also belong to the same cluster in the hierarchical cluster tree and show mutually informative expression in the fetal brain of males and females based on the mutual information analysis.

Further analysis identified that several DEGs were markers of specific cell types in the brain. Recently, single-cell RNA sequencing studies have identified cell types and cell-specific marker genes in the human and mouse brain, curated in the PanglaoDB database (Franzén et al., 2019). Those marker genes and the associated cell types were downloaded to perform gene orthology analysis to explore if any of those known brain cell markers were orthologous to the genes differentially expressed in the pig fetal brain in the current study. 357 significant DE genes were identified that have also been identified as marker genes of neurons, oligodendrocytes, ependymal cells, astrocytes, and 
Schwann cells. DEGs that are markers of neurons and oligodendrocytes were relatively more abundant than markers of other known cell types of brain (Figure 2.7A). Those marker genes were predominantly found as significant DEGs in the edgeR analysis between $\mathrm{d} 60$ and $\mathrm{d} 90$ with relative greater numbers in the male than female brain (Figure 2.7B). Expression patterns of these marker genes were analyzed for specific cell types, for example neurons and astrocytes. They showed variable patterns in the male and female brain during development from d45 to 60 and from d60 to 90 (Figure 2.8). During development in the male brain from d60 to d90, the mean expression level of the marker genes of neurons as well as astrocytes was relatively lower than that of females, possibly indicating a sexual dimorphic functional role of those cells in the fetal brain of pigs.

Comparison of gene expression in the fetal brain and placenta

The gene expression data of the fetal pig brain on d60 and d90 from the current study was pooled, regardless of fetal sex, and used to compare with gene expression data previously generated from the pig placenta (accession number GSE110414) on d60 and d90 (Wang et al., 2019). This comparative analysis identified genes that were expressed either in the placenta or the fetal brain of pigs in an 'ON/OFF' coordinated manner. A gene was considered 'expressed' if the FPKM value was $>10$ and a gene with FPKM $<1$ was considered 'not expressed'. This study did not further examine a gene with FPKM $\geq$ 1 but $\leq 10$. On d60, 553 genes were expressed in the fetal brain but not in the placenta, whereas 87 genes were expressed in the placenta but not in the fetal brain. On d90, 554 genes were expressed in the fetal brain but not in the placenta and 80 genes were expressed in the placenta but not in the fetal brain. This study identified 45 of these 
ON/OFF genes between the placenta and the fetal brain were related to ion transporters, suggesting that ion transporter genes play a role in the feto-placental communication and circulation, possibly by regulation of the placental-blood barrier (Cousins and Wood, 2010; Hosoya and Tachikawa, 2011; Nigam et al., 2015; St-Pierre et al., 2004; Sugiyama et al., 2003).

\section{Discussion}

This study provides insights into sex differences regulating brain development in fetal pigs. It is known that the rate of growth of male and female fetuses varies throughout gestation (Stenhouse et al., 2019). In the present study, fetal growth rate per day increased by nearly 3 times from d60 to $\mathrm{d} 90$ compared to $\mathrm{d} 45$ to $\mathrm{d} 60$. Although in the current study there was no observed difference in the body weight of male compared to female fetuses, birth weight is generally higher in male than female piglets (Baxter et al., 2012). Sex-mediated differences in growth are well documented in other animals too, including mice and humans (Crawford et al., 1987; Dhakal et al., 2020; Galjaard et al., 2019; Lampl et al., 2010; Tekola-Ayele et al., 2019).

Results from the RNA-seq analysis showed that fewer DEGs in the male versus female fetal brain were identified in earlier (d45 and d60) compared to later (d60 and d90) gestation. The differences in DEGs suggests that brain development is different in male and female pig fetuses and that there are changes in the brain development as gestation progresses, a finding that is consistent with an earlier report (McPherson et al., 2004). Overall, there was approximately a 3.5 -fold increase in the number of genes that were altered between the $\mathrm{d} 60$ and $\mathrm{d} 90$ timepoints compared to between $\mathrm{d} 45$ and $\mathrm{d} 60$ 
(Table 2.1). A greater number of DEGs in late gestation is suggestive of preparation of the brain for postnatal functions (Rabaglino et al., 2014). The homeobox (HOX) A5 gene that regulates pattern formation in early development (Lewis et al., 1999; Taylor et al., 1997) was in the top upregulated DEGs between d45 to d60 in fetuses of both sexes. $H O X B 5$ and $D 3$ genes were also found in the top upregulated genes between $\mathrm{d} 45$ and d60 of fetal brain of females, but not in males. Of note, the development of the female reproductive tract seems to be partially directed by HOX genes (Taylor et al., 1997). The myelin associated glycoprotein $(M A G)$ was among the most downregulated DEGs in both male and female fetuses from d60 to 90 and it is a myelin-expressed cell-adhesion and bidirectional signaling molecule (Pronker et al., 2016). MAG maintains the myelin-axon spacing by interacting with specific neuronal glycolipids, inhibiting axon regeneration, and controlling myelin formation (Pronker et al., 2016). Similarly, the gene coding for $E R M N$, which plays a role in cytoskeletal rearrangements and CNS myelination in humans and mice (Brockschnieder, 2006), was one of the top downregulated DEGs between $\mathrm{d} 60$ and $\mathrm{d} 90$ in both males and females.

In litter-bearing mammals, sex of neighboring fetuses in the uterus influences the hormonal environments of fetal development to confound sex-biased traits in the offspring (Ryan and Vandenbergh, 2002). A female fetus developing between two males show masculinized anatomical and physiological traits (reviewed by vom Saal, 1989). Similarly, a fetus positioned between females have greater concentrations of estradiol than a fetus positioned between male litter mates, which can lead to feminization of males as adults (reviewed by vom Saal, 1989). In pigs, the effect of intrauterine position and fetal sex was investigated (Rohde Parfet et al., 1990), but there was no effect of fetal 
position in the uterus on birth weight or survivability of piglets. However, a subsequent study (Jang et al., 2014) found that fetuses on each end of the uterine horn grow faster than the fetuses on the medial side of the uterus and further suggested that sex of the adjacent fetuses has lesser influence than the absolute intrauterine position on fetal growth in pigs. Given this premise in the literature, this study wanted to determine if sex of the adjacent fetuses had an influence on gene expression of the fetal brain. In this study, male fetuses that were flanked by two female fetuses (M.F.F) or by a male fetus on the medial side and a female fetus on the lateral side (M.M.F) were identified. Gene expression of the developing brain of those males showed significant changes in expression of 54 genes, suggesting that gene expression in fetal brain is impacted by sex of the adjacent fetuses. Though this study did not include age matched female fetuses that varied in the sex of on the medial side, it was able to generate age matched (d45 and 90) female fetuses that had difference in sex of the fetus on the lateral side. However, differential expression analysis showed no significant difference in the gene expression of the fetal brain. It is plausible that there is a stronger impact on male fetuses than female fetuses. It may also be that sex of the medial fetus only influences gene expression of the fetal brain, and the fetus on the lateral side has no effect. Only 18 fetal brains were sequenced in this study which posed a shortcoming in the current study to fully address this important question if both the medial and lateral fetus impacted gene expression of male and female fetuses. A future study using a larger sample size of fetal collections will reveal better clarity to this issue.

This study revealed a key observation concerning the regulation of ion transporter genes in the fetal brain during development. A comparative analysis of gene expression 
data at $\mathrm{d} 60$ and $\mathrm{d} 90$ identified 45 ion transporter genes that were coordinately regulated in the form of ON/OFF expression between the fetal brain and placenta of pigs. Ion transporters play crucial roles in fetal development (Jansson et al., 2009; Nigam et al., 2015; Sangkhae and Nemeth, 2019; Sugiyama et al., 2003). They not only control the placental-blood barrier but also modulate feto-placental circulation and molecular communication (Nigam et al., 2015; Sangkhae and Nemeth, 2019). Interestingly, the analysis of effect of intrauterine fetal location on the brain also suggested that ion transporters are involved in the influence of the medial fetal sex on fetal brain development. The effect of adjacent fetuses on brain development is thought to be modulated by sex dependent maternal resource sharing by the litter via placental matrotrophy (Ryan and Vandenbergh, 2002; vom Saal, 1989). In this context, these findings suggest that ion transporters may be involved in sex-biased regulation of the brain-placental axis. This finding is consistent with earlier reports that transporter genes, including the solute carrier transporter gene, play influential roles in the regulation of the brain placental axis (Behura et al., 2019a; Behura et al., 2019b; Rosenfeld, 2021).

\section{Conclusion}

In conclusion, these findings provide insights into the molecular regulation of fetal brain development and provide data for better understanding the influence of fetal sex and fetal localization in the uterus on gene expression during brain development. This study sets the foundation that large animals, such as pigs, can be used as models to better understand regulation of brain development during fetal stages. 
Table 2.1. The number of significantly differentially expressed exons (upregulated or downregulated) in the developing male (M) and female $(F)$ fetal brain of pigs at gestation day (d) 45, 60 and 90.

\begin{tabular}{|l|l|l|}
\hline \multicolumn{1}{|c|}{$\begin{array}{c}\text { Comparison } \\
\text { (group1 vs. group2) }\end{array}$} & Number of DEGs & \multicolumn{1}{|c|}{$\begin{array}{c}\text { Differential } \\
\text { expression (group1 } \\
\text { relative to group2) }\end{array}$} \\
\hline F.d45 vs. F.d60 & 801 & Upregulated \\
\hline F.d45 vs. F.d60 & 1258 & Downregulated \\
\hline F.d60 vs. F.d90 & 2665 & Upregulated \\
\hline F.d60 vs. F.d90 & 2660 & Downregulated \\
\hline M.d45 vs. M.d60 & 571 & Upregulated \\
\hline M.d45 vs. M.d60 & 752 & Downregulated \\
\hline M.d60 vs. M.d90 & 3163 & Upregulated \\
\hline M.d60 vs. M.d90 & 3442 & Downregulated \\
\hline
\end{tabular}


Table 2.2. The number of significantly differentially expressed exons (upregulated or downregulated) in the developing male (M) and female (F) fetal brain of pigs at gestation day (d) 45, 60 and 90 .

\begin{tabular}{|l|l|l|l|l|}
\hline & F.d45 - F.d60 & F.d60 - F.d90 & M.d45 - M.d60 & M.d60 - M.d90 \\
\hline Downregulated & 132 & 694 & 36 & 274 \\
\hline Upregulated & 205 & 268 & 14 & 1772 \\
\hline
\end{tabular}


Table 2.3. PANTHER pathways that were significantly over-represented by the differentially expressed genes (DEGs) in the developing male (M) and female (F) fetal brain. Fold enrichment, false discovery rate (FDR), sex and the gestation days compared for DEGs are shown.

\begin{tabular}{|c|c|c|c|c|c|}
\hline Pathway & $\begin{array}{l}\text { Number of } \\
\text { DEGs }\end{array}$ & $\begin{array}{l}\text { Fold } \\
\text { Enrichment }\end{array}$ & FDR & Sex & Gestation days \\
\hline $\begin{array}{l}\text { Ionotropic glutamate receptor } \\
\text { pathway (P00037) }\end{array}$ & 15 & 3.32 & $7.40 \mathrm{E}-03$ & $\mathrm{~F}$ & $d 45-d 60$ \\
\hline p53 pathway (P00059) & 20 & 2.49 & $1.90 \mathrm{E}-02$ & $\mathrm{~F}$ & $\mathrm{~d} 45-\mathrm{d} 60$ \\
\hline $\begin{array}{l}\text { Endothelin signaling pathway } \\
\text { (P00019) }\end{array}$ & 19 & 2.47 & $2.30 \mathrm{E}-02$ & $\mathrm{~F}$ & d45 - d60 \\
\hline $\begin{array}{l}\text { Gonadotropin-releasing hormone } \\
\text { receptor pathway (P06664) }\end{array}$ & 48 & 2.3 & $1.30 \mathrm{E}-04$ & $\mathrm{~F}$ & $\mathrm{~d} 45-\mathrm{d} 60$ \\
\hline Angiogenesis (P00005) & 35 & 2.21 & $3.90 \mathrm{E}-03$ & $\mathrm{~F}$ & $d 45-d 60$ \\
\hline Wnt signaling pathway (P00057) & 52 & 1.81 & $7.40 \mathrm{E}-03$ & $\mathrm{~F}$ & $d 45-d 60$ \\
\hline $\begin{array}{l}\text { Gonadotropin-releasing hormone } \\
\text { receptor pathway (P06664) }\end{array}$ & 99 & 1.82 & $1.60 \mathrm{E}-04$ & $\mathrm{~F}$ & d60 - d90 \\
\hline Angiogenesis (P00005) & 68 & 1.65 & $4.00 \mathrm{E}-02$ & $\mathrm{~F}$ & d60 - d90 \\
\hline Wnt signaling pathway (P00057) & 30 & 1.99 & $3.40 \mathrm{E}-02$ & M & $d 45-d 60$ \\
\hline $\begin{array}{l}\text { Histamine } \mathrm{H} 1 \text { receptor mediated } \\
\text { signaling pathway }(\mathrm{P} 04385)\end{array}$ & 27 & 2.13 & 4.80E-02 & M & d60 - d90 \\
\hline $\begin{array}{l}\text { Endothelin signaling pathway } \\
\text { (P00019) }\end{array}$ & 48 & 1.96 & $1.10 \mathrm{E}-02$ & M & d60 - d90 \\
\hline $\begin{array}{l}\text { Metabotropic glutamate receptor } \\
\text { group III pathway (P00039) }\end{array}$ & 39 & 1.93 & 4.00E-02 & M & d60 - d90 \\
\hline $\begin{array}{l}\text { Heterotrimeric G-protein signaling } \\
\text { pathway-Gq alpha and Go alpha } \\
\text { mediated pathway (P00027) }\end{array}$ & 67 & 1.88 & $3.00 \mathrm{E}-03$ & M & d60 - d90 \\
\hline Angiogenesis (P00005) & 88 & 1.75 & $3.20 \mathrm{E}-03$ & M & d60 - d90 \\
\hline $\begin{array}{l}\text { Heterotrimeric G-protein signaling } \\
\text { pathway-Gi alpha and Gs alpha } \\
\text { mediated pathway (P00026) }\end{array}$ & 81 & 1.69 & $6.50 \mathrm{E}-03$ & M & d60 - d90 \\
\hline $\begin{array}{l}\text { EGF receptor signaling pathway } \\
\text { (P00018) }\end{array}$ & 65 & 1.6 & $5.00 \mathrm{E}-02$ & M & d60 - d90 \\
\hline CCKR signaling map (P06959) & 76 & 1.53 & $4.50 \mathrm{E}-02$ & $\mathrm{M}$ & d60 - d90 \\
\hline $\begin{array}{l}\text { Gonadotropin-releasing hormone } \\
\text { receptor pathway (P06664) }\end{array}$ & 102 & 1.53 & $1.20 \mathrm{E}-02$ & M & d60 - d90 \\
\hline
\end{tabular}


Figure 2.1. The weight $(\mathrm{g})$ of male $(\mathrm{M})$ and female $(\mathrm{F})$ pig fetuses $(\mathbf{A})$, the weight $(\mathrm{g})$ of fetal brains $(\mathbf{B})$, the relative weigh of the fetal brain (brain weight per 100g of fetal weight) $(\mathbf{C})$ and the length $(\mathrm{cm})$ of fetal brains (D) on gestation day (d) 45, 60 and 90 .

A

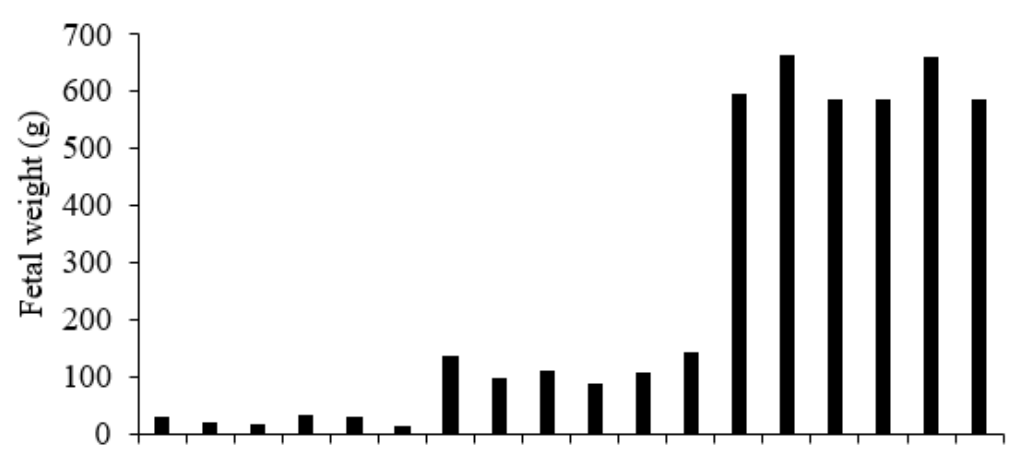

$\stackrel{\omega}{\infty}$

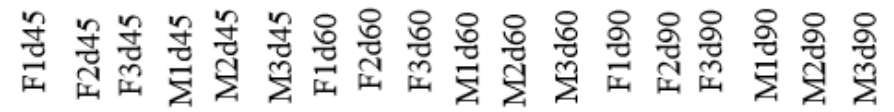

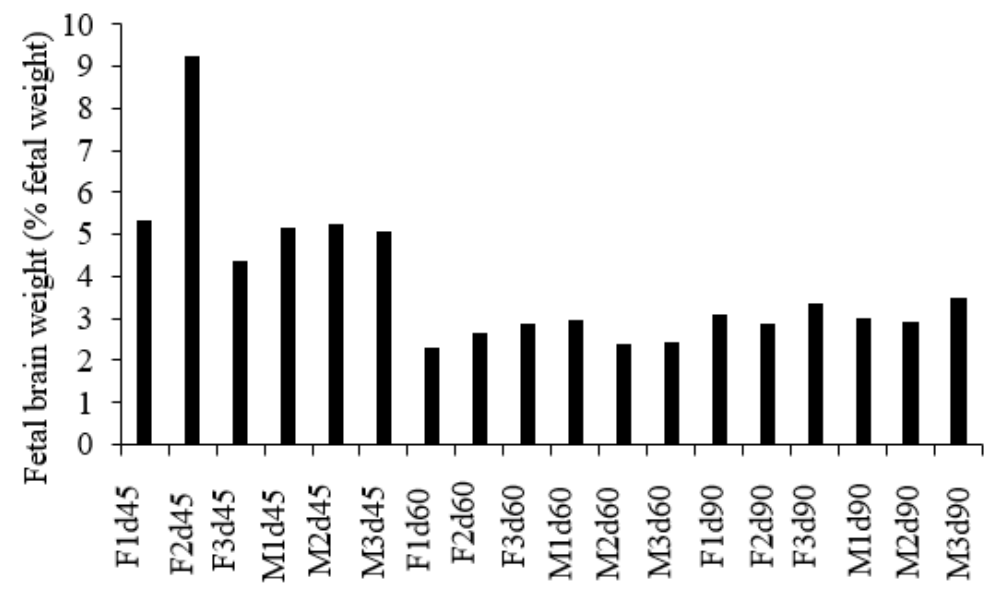

B
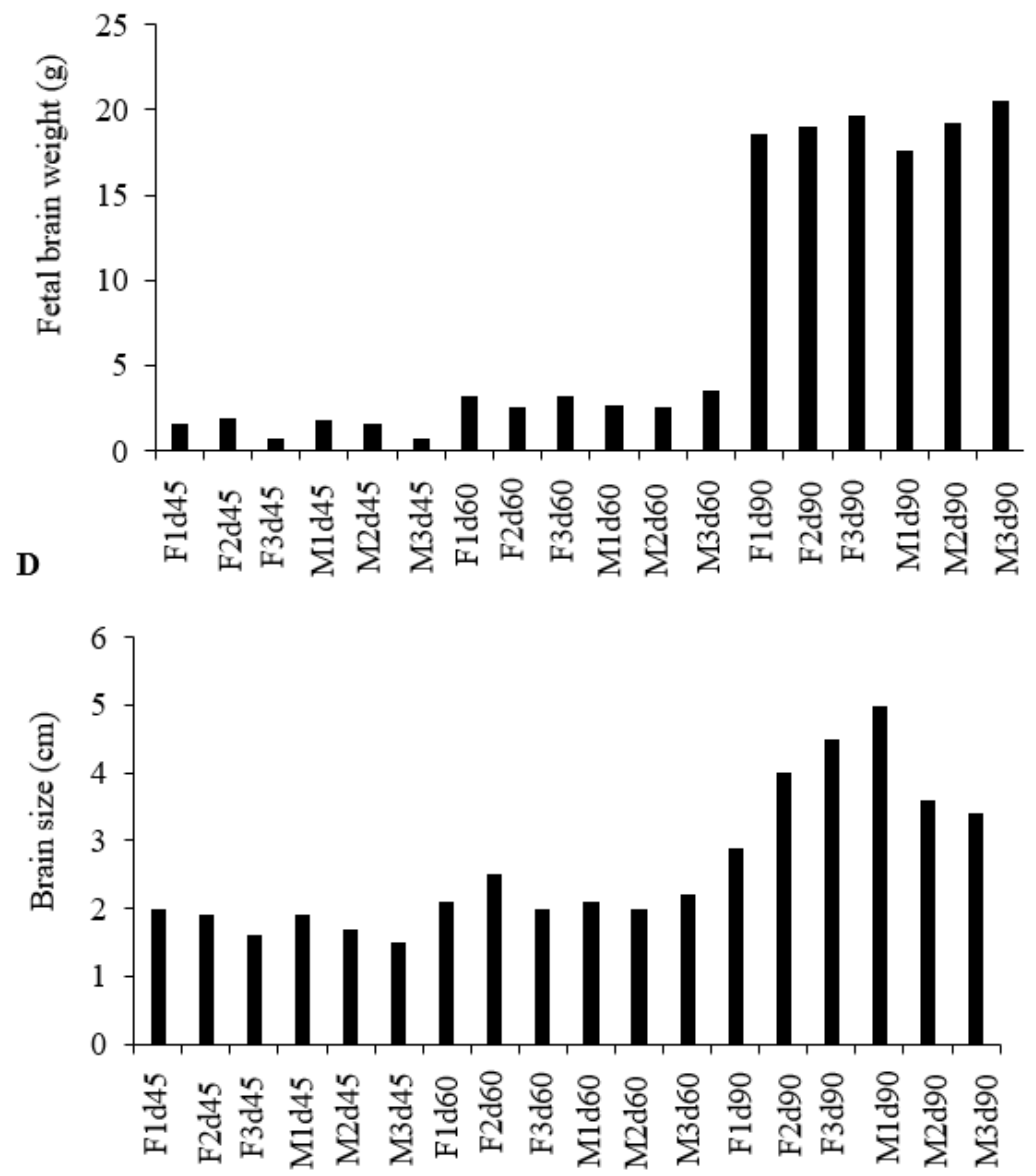
Figure 2.2. A. A principal component analysis of the gene expression variation of male $(\mathrm{M})$ and female (F) fetal brain on gestation day (d) 45, 60 and 90. The dotted line ellipse shows the group representing gene expression of fetal brain on day 45 and 60, and the solid line ellipse shows the group representing gene expression of fetal brain on day 90 . B. A hierarchical clustering of gene expression variation also shows that fetal brain gene expression pattern on d90 is different from that on d45 and 60 . The scale of branch height is shown to the left of the cladogram.

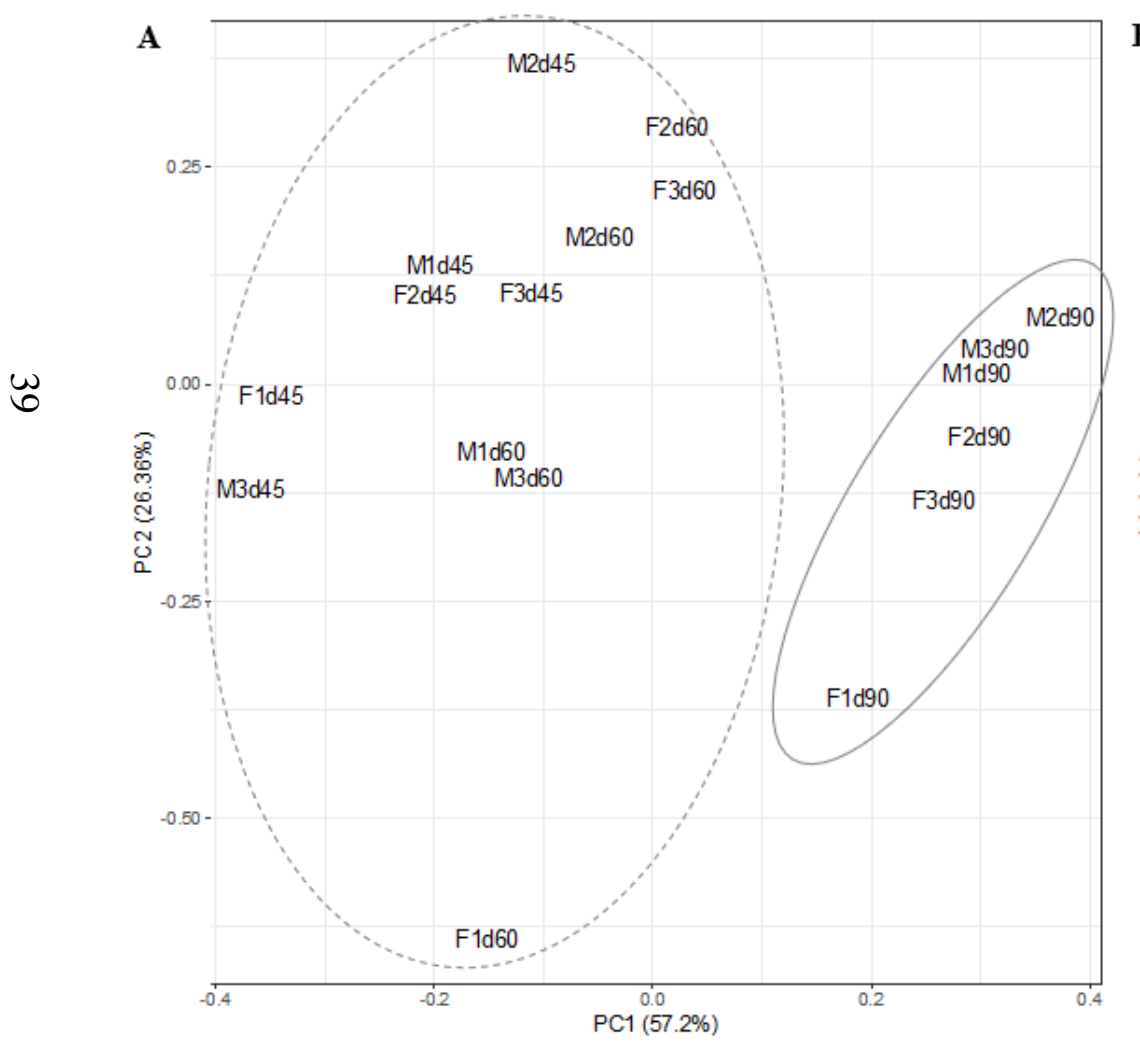

B

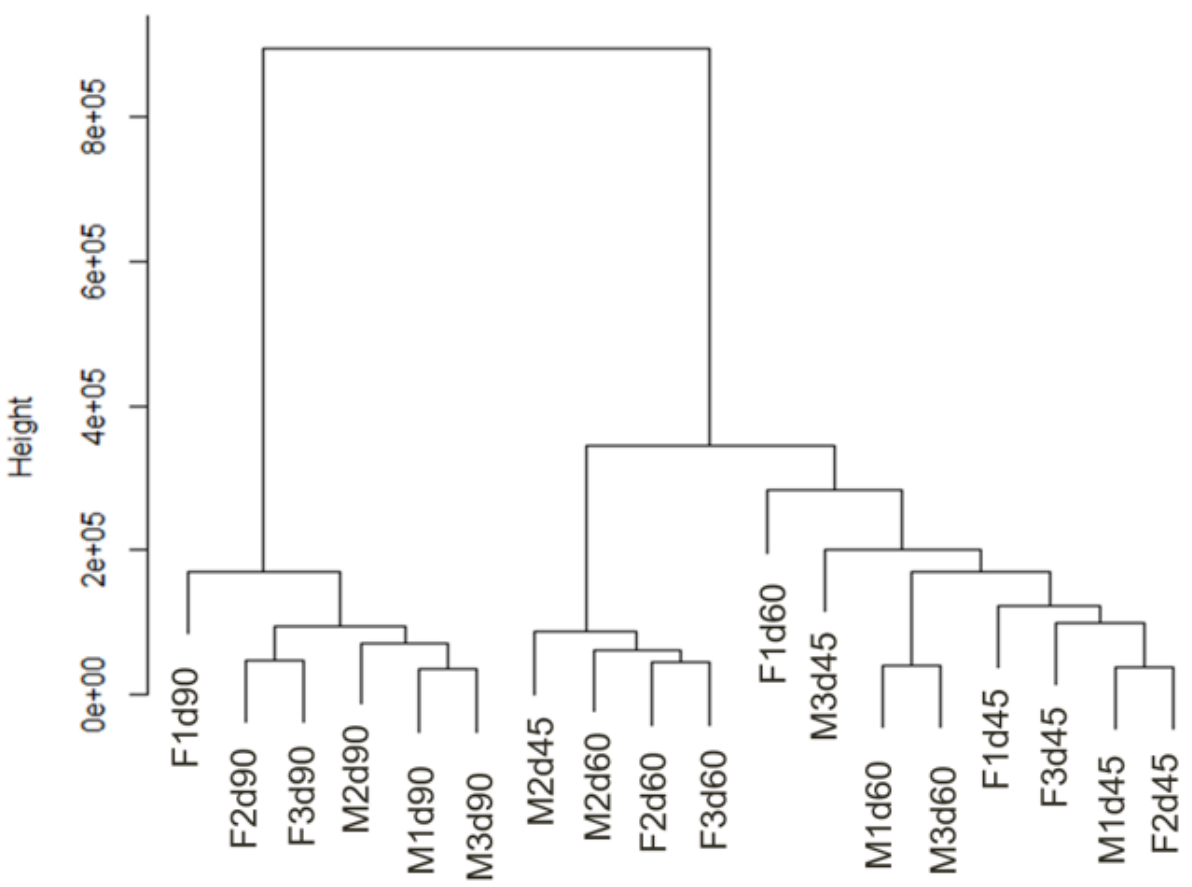


Figure 2.3. A. A chord diagram showing the mutation information pattern of gene expression relationship between the fetal brain of males (M) and females (F) on gestation day (d) 45, 60 and 90 (sample names on the circumference of the circular layout plot). The arcs show the connectedness between samples based on adjacent matrix of mutation information scores. The scale of data variation for each sample is shown on the circumference. B. A tanglegram comparing clusters of the top 20 expressed genes in the pig fetal brain based on their expression patterns in males and females during development. The colored tangles connecting nodes of both cluster trees show common sub-clusters of genes in both males and females. The scales below the plots show units of the branch length variation.

$\mathbf{A}$

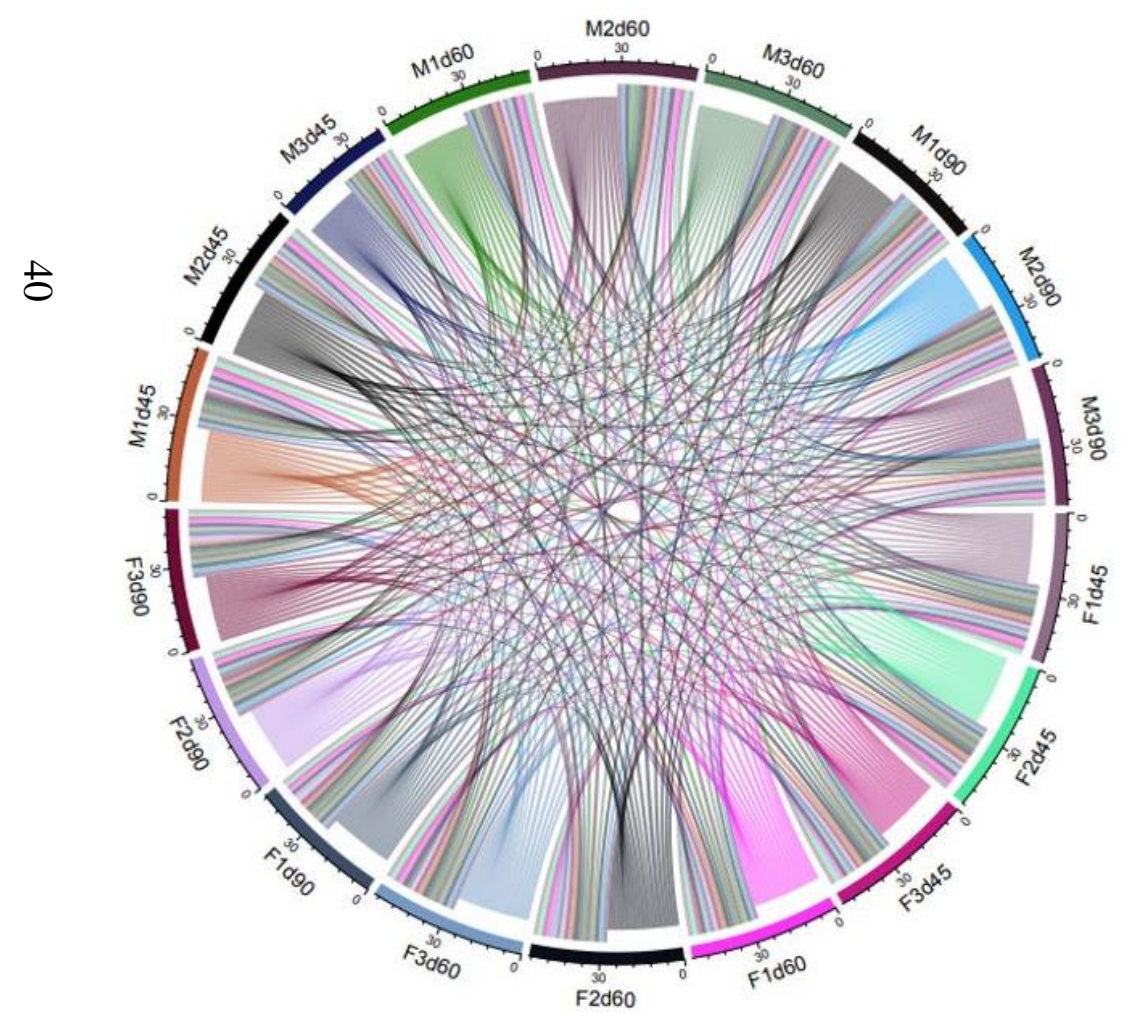

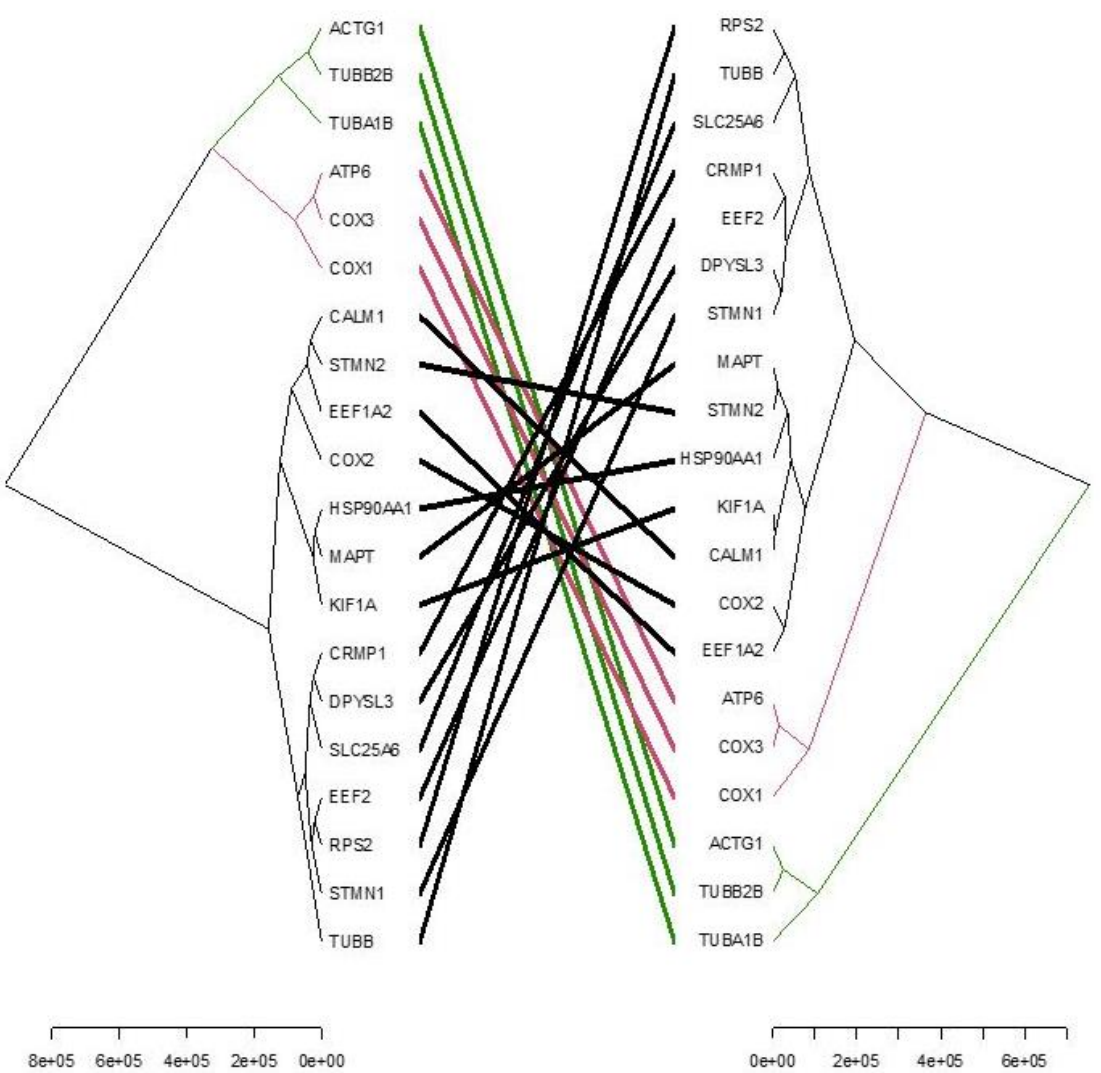


Figure 2.4. Volcano plots showing differential expression of genes in female $(\mathrm{F})$ fetal brain between gestation day (d) 45 - d60 (A), male (M) fetal brain between d45 - d60 (B), female fetal brain between $\mathrm{d} 60$ - $\mathrm{d} 90(\mathbf{C})$, and male fetal brain between $\mathrm{d} 60$ - d90 (D). In each plot, the $y$-axis shows the $-\log 10(\mathrm{FDR})$ (false discovery rate) values and $\mathrm{x}$-axis shows log fold change (FC) values. The orange color shows genes that were downregulated, and blue color shows genes upregulated between the two groups in each plot. The horizontal line above value 0 in axis represent the FDR value of 0.05 , the value used to identify significance of differential expression of genes.

A $\quad$ F.d45 - F.d60

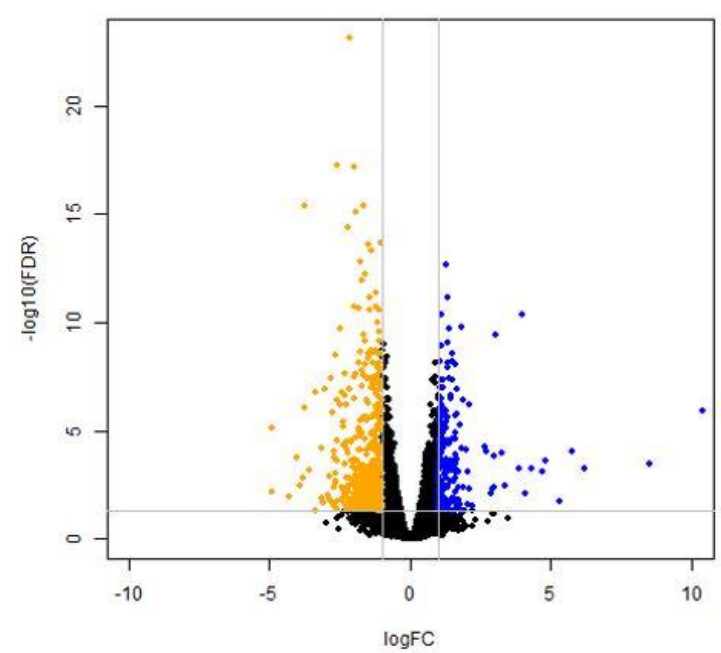

C M.d45 - M.d60

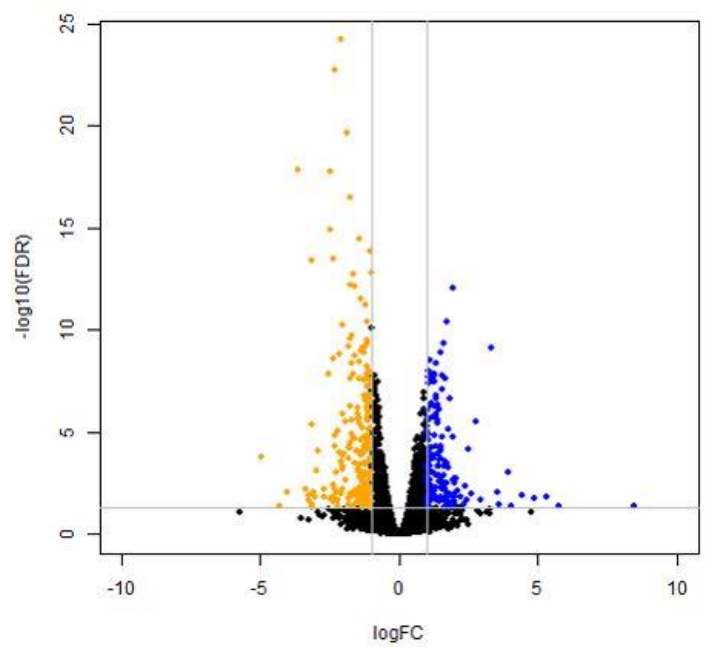

B

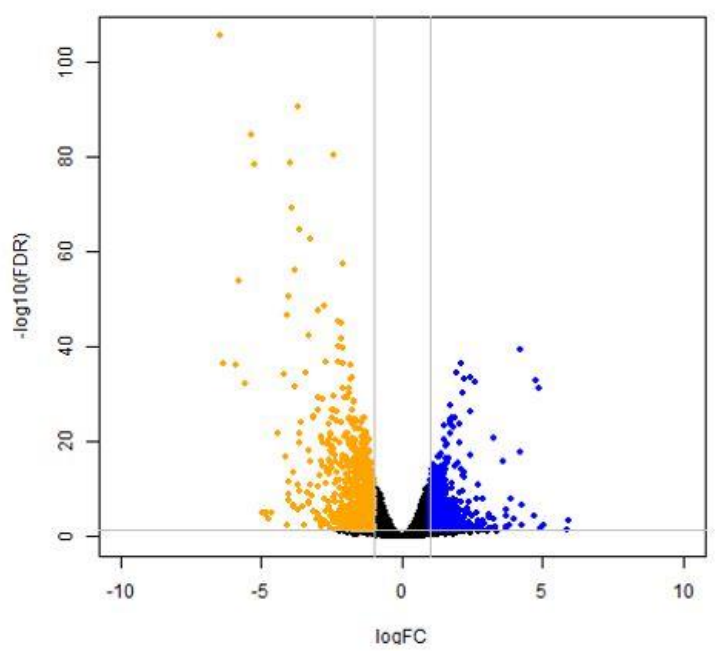

D M.d60 - M.d90

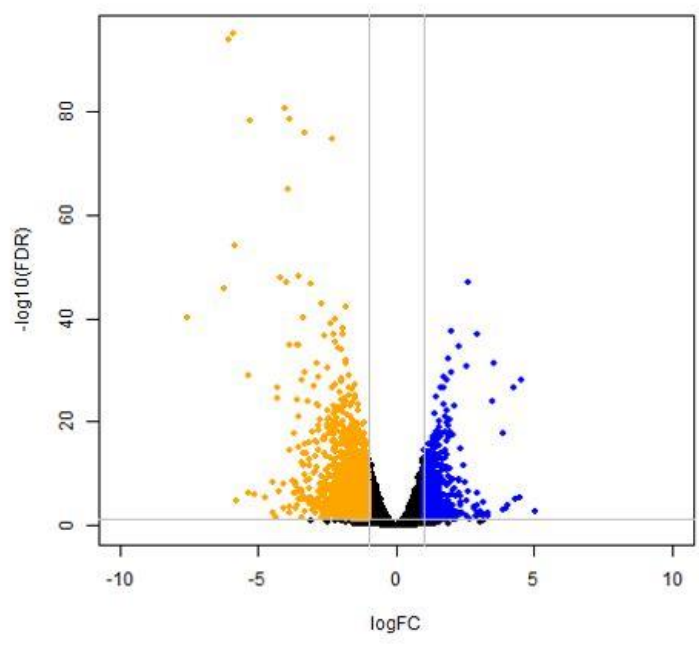


Figure 2.5. Venn diagrams showing the number of upregulated (Up) and downregulated (Down) genes between gestation day (d) 45 60 and $\mathrm{d} 60$ - d90 in females (A) and males (B). The shades indicate the proportion to gene counts.
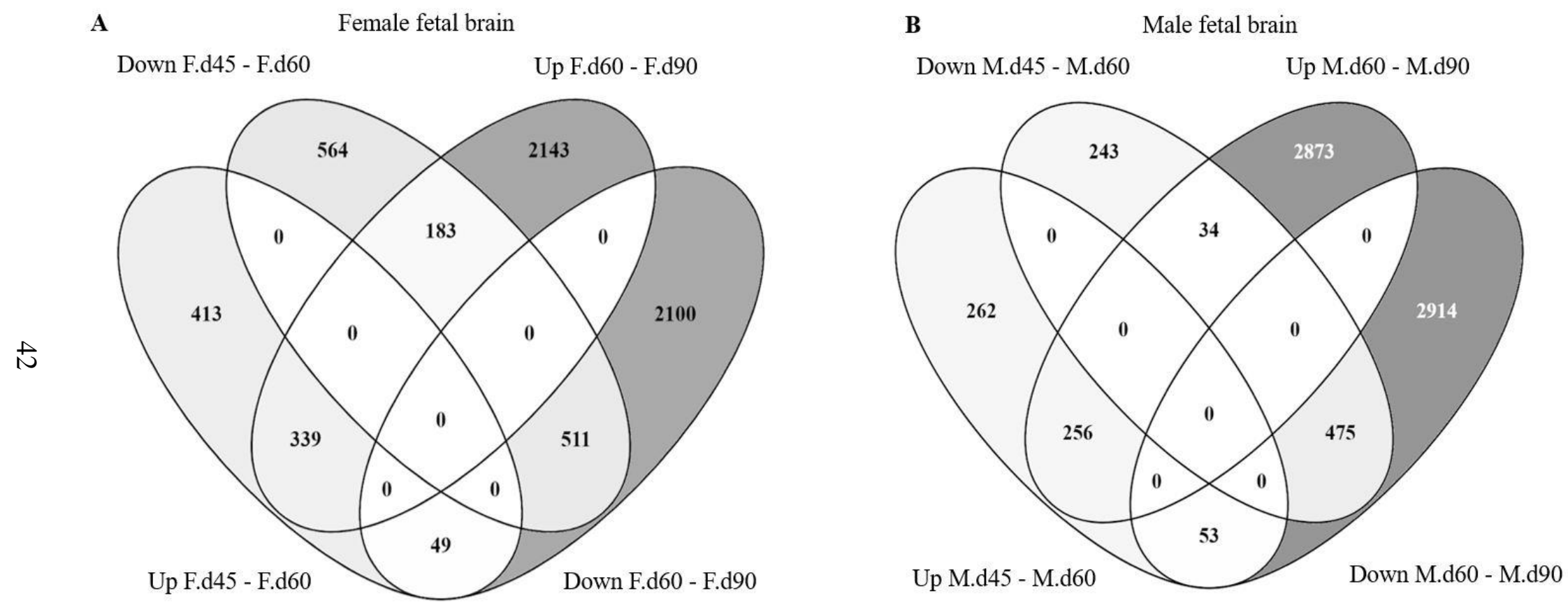
Figure 2.6. Network plots of genes based on mutation information of the expression changes during development of the female fetal brain (A) and male fetal brain (B). The lines (edges) connecting the nodes show how a gene is transcriptionally interconnected with other genes. The pink color shows the genes in the female brain and the blue color shows the genes in the male brain.

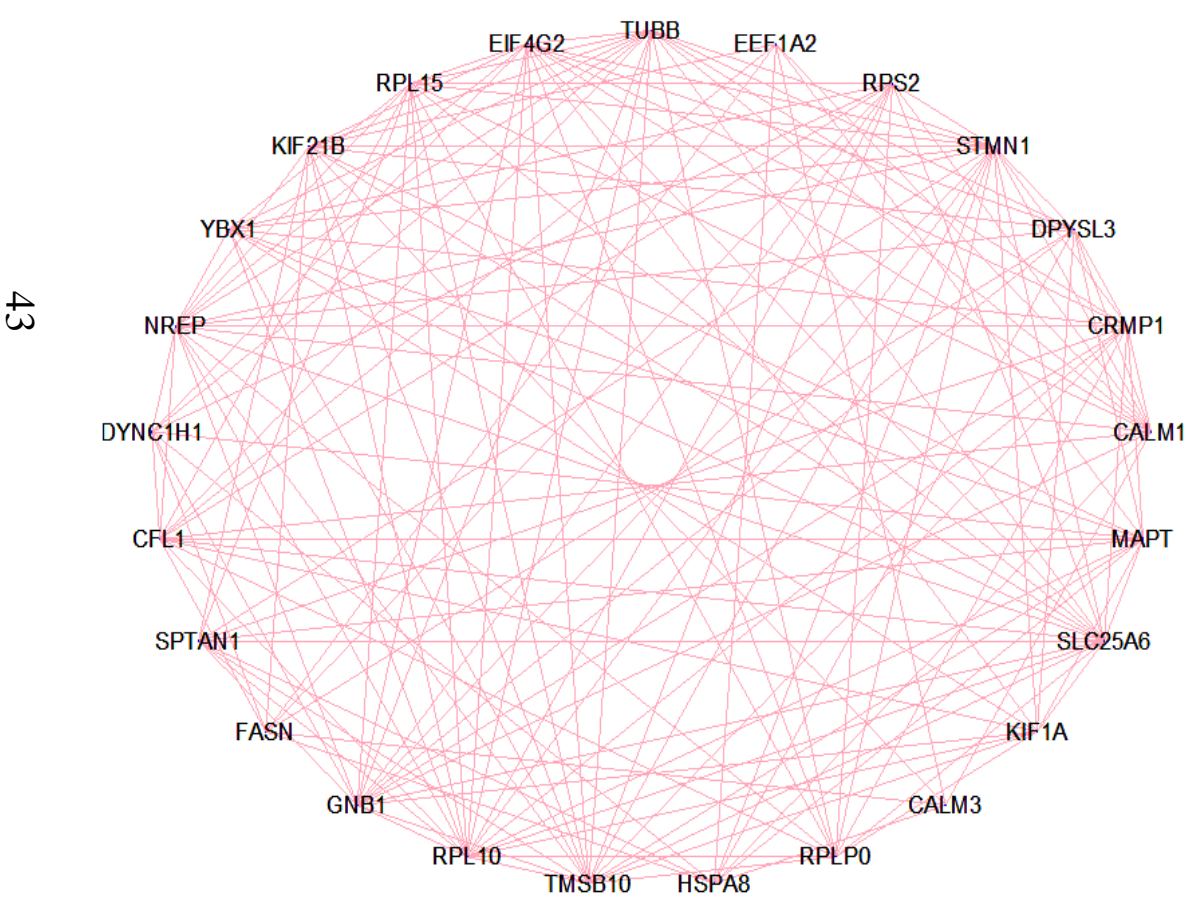

B

Male brain

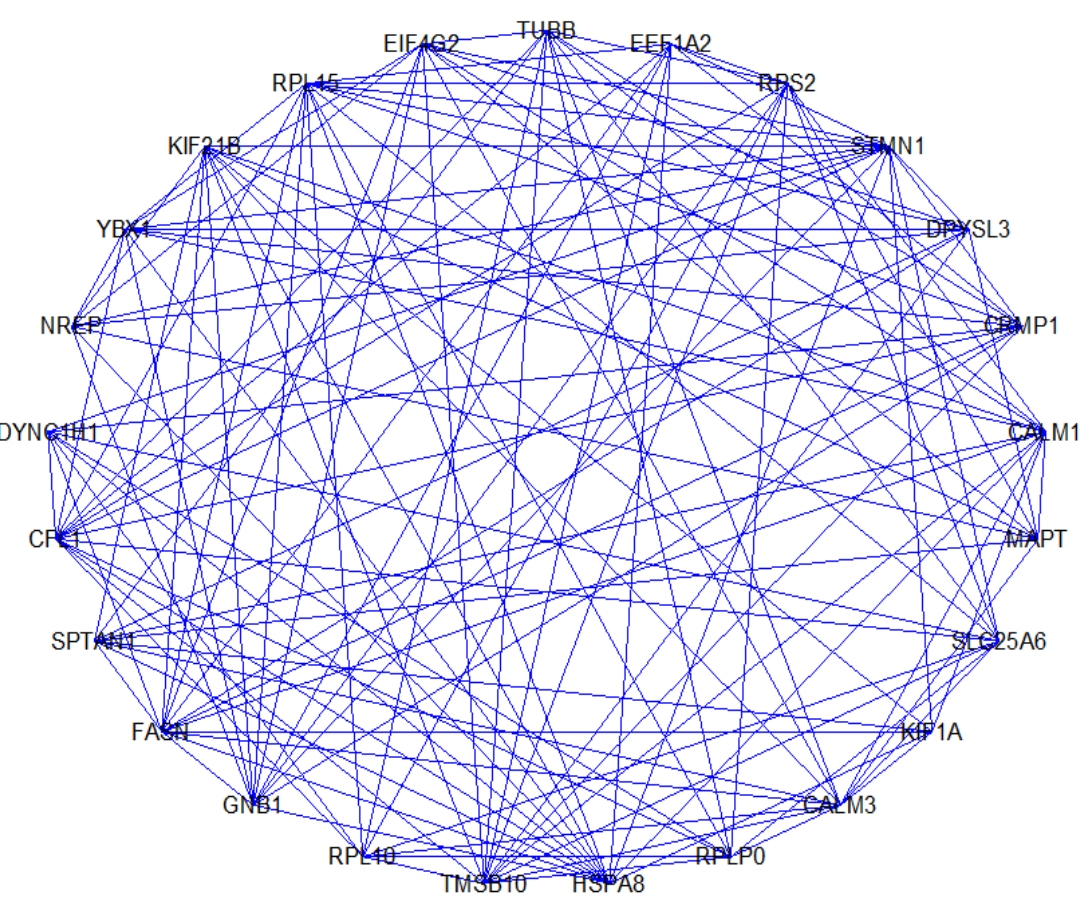


Figure 2.7. A. A donut plot showing the proportion of marker genes representing different brain cells (color coded). B. A heatmap showing the number markers genes associated with different cell types in the brain (shown in rows) and the developmental time periods (gestation day (d) 45, 60 and 90) during which those genes were significantly differentially expressed in the male (M) and female (F) fetal brain (shown in columns). The cluster patterns of rows and columns are shown along with the color scale (to the right).

A

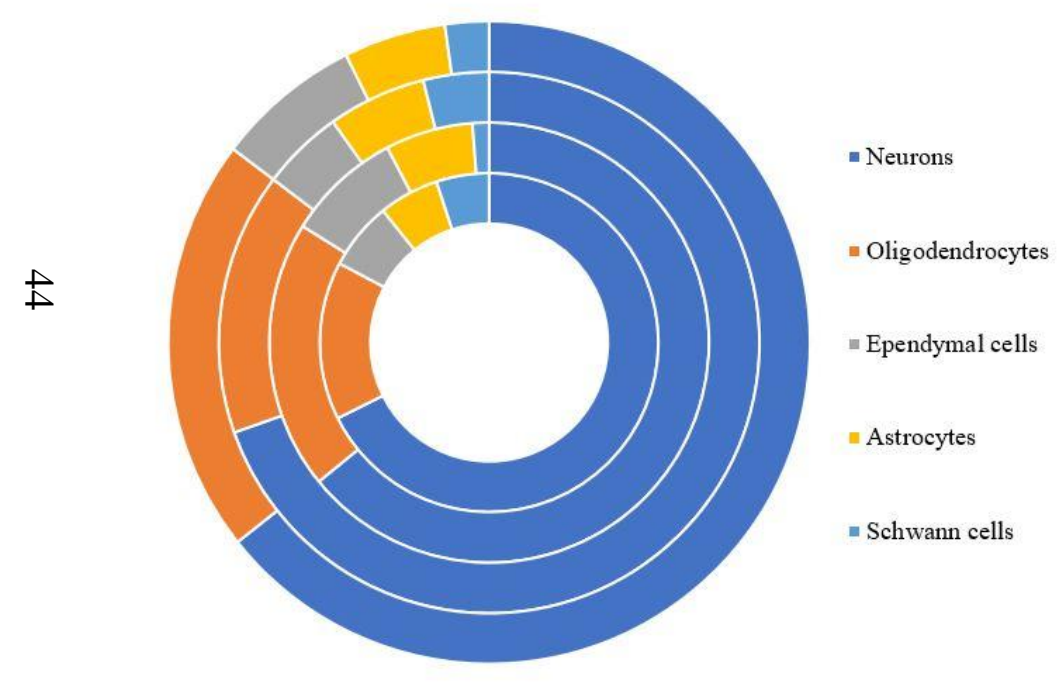

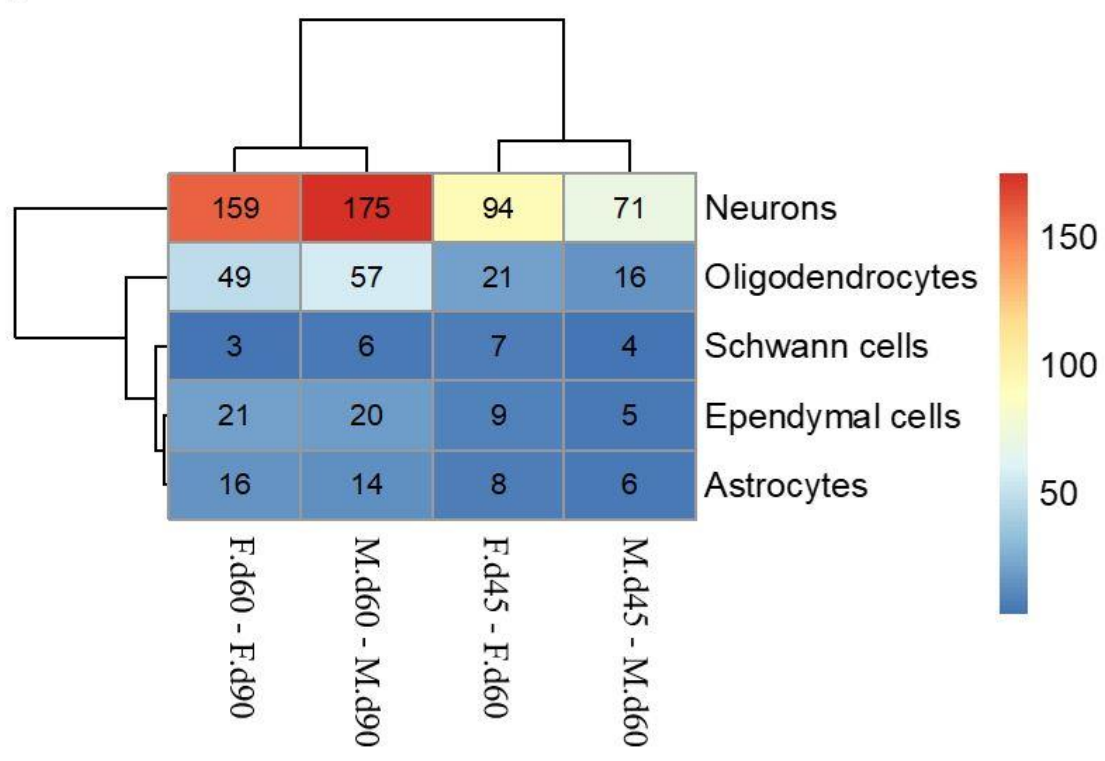


Figure 2.8. Violin plots of expression changes in marker genes specific to neurons $(\mathbf{A})$ and astrocytes $(\mathbf{B})$ of male $(\mathrm{M})$ and female $(\mathrm{F})$ fetal brain on gestation day (d) 45 to 60 and then d60 to d90. In both plots, the values on the y-axis represent the log fold changes determined from egdeR between gestation day in male and female brain (shown in $\mathrm{x}$-axis). The plots representing the female brains are shown in pink, and those of the male brains are shown in blue.

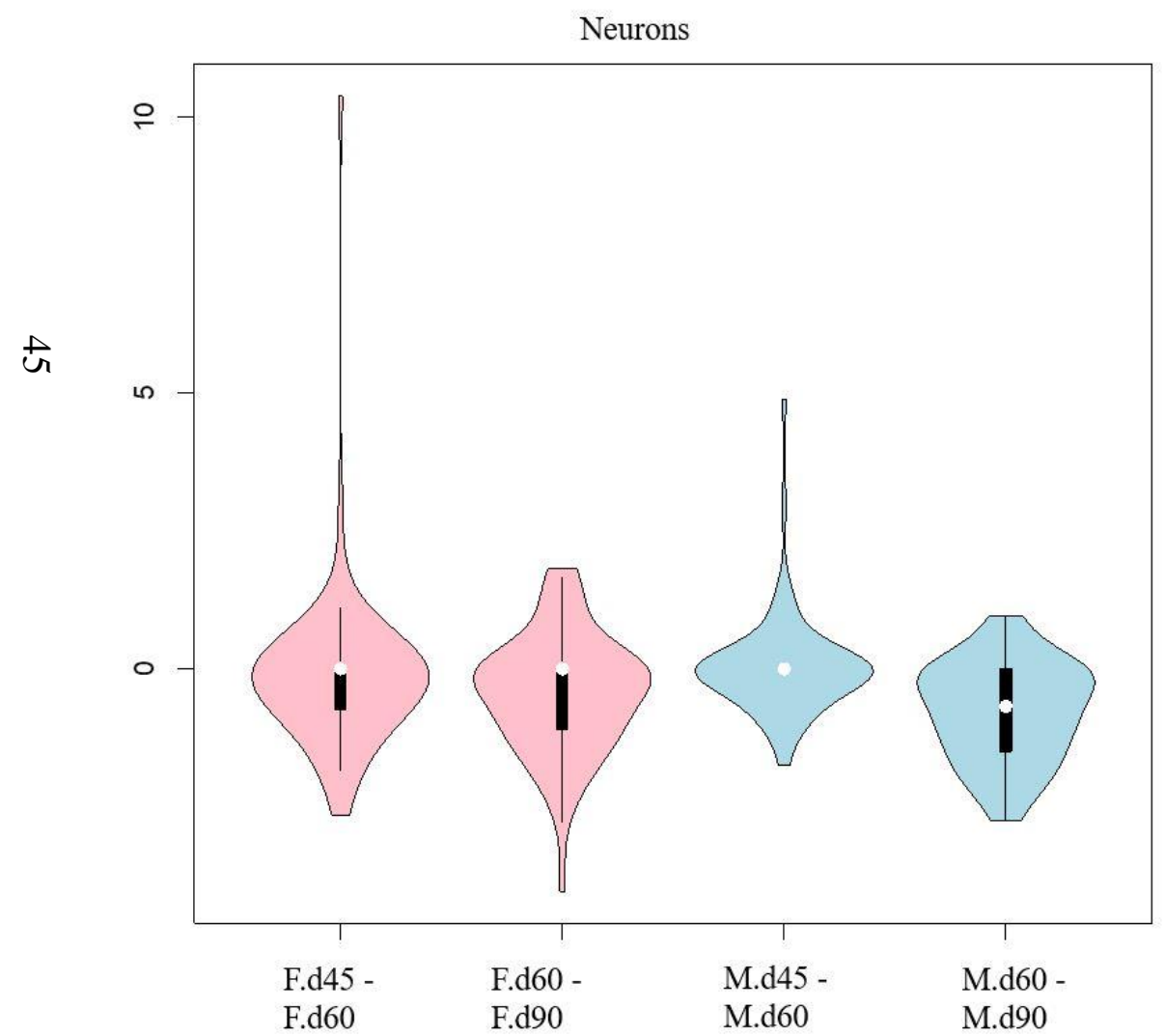

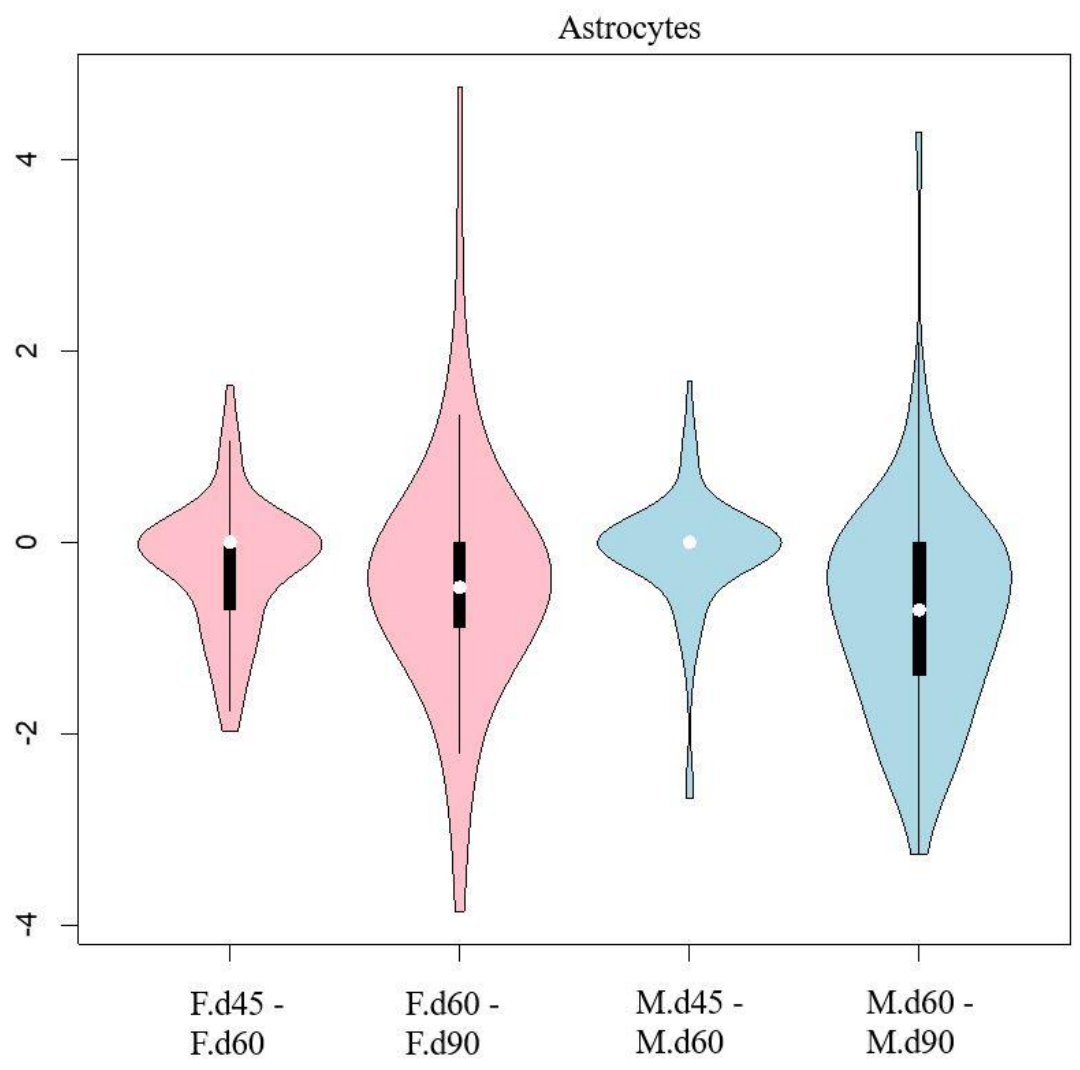




\section{Chapter 3}

\section{Epigenetic changes during fetal brain development in pigs}

\section{Abstract}

The aim of this study is to investigate DNA methylation in pigs. It was hypothesized that specific genes are epigenetically controlled during fetal brain development. To test this hypothesis, DNA methylation in male $(\mathrm{M})$ and female $(\mathrm{F})$ fetal brains at gestation day (d) 45, 60 and 90 was profiled. These time points are the same as those used to profile gene expression of the fetal brain (Chapter 2) and the purpose is to compare DNA methylation and gene expression to determine if there is an association between the two. The DNA methylation profiling was performed using the enzymatic methyl sequencing (EM-seq) approach, followed by Bismark and edgeR analysis to identify differential methylation sites (DMSs) in the developing fetal brain. There were 3,525,376 methylations in total across the three gestation days of the developing fetal brain, however, hotspots of methylation were observed in specific chromosomal regions. Gene Ontology (GO) analysis found significant association of specific developmental related processes with the genes positionally closest to the methylation sites in the fetal brain. This analysis showed contrasting patterns in gene expression and DNA methylation variation during development of the fetal brain. Variation in gene expression increased, whereas that of methylation decreased, as the fetal brain developed from d45 through d90. The analysis also showed differences in DNA methylation in the X-chromosome occurred more frequently in the brain of female fetuses compared to males. Methylation sites were mapped to known single nucleotide polymorphisms (SNPs) in the pig genome and this identified a disproportionately greater number of methylations of genetic variants 
in the X-chromosome of the female fetal brain $(n=5,120)$ than the male fetal brain $(\mathrm{n}=640)$. A key finding of this study is the canonical correlation between methylation specific sites between the fetal pig brains and blood from adult pigs. 1,475 sites in the pig genome were identified that were methylated in the fetal brain, irrespective of sex, during development. The same sites were methylated in a canonically correlated manner in the adult blood, both in sows and boars. The findings from this study show that there were sites where there were concordances in methylation between the fetal brain and adult blood. As methylation in blood occurs in an age correlated manner, this finding suggests that epigenetic changes that occur during development of the fetal brain are predictive of aging. This is consistent with the Dilman theory of developmental aging (DevAge), which suggests that aging and early development of the brain are regulated by common molecular processes.

\section{Introduction}

The domesticated pig, Sus scrofa, is used as an animal model to study the human brain relating to developmental disorders and neurodegenerative diseases (Hoffe and Holahan, 2019; Kinder et al., 2019; Lind et al., 2007; Mudd and Dilger, 2017). Having a gyrencephalic brain similar to that of humans (Villadsen et al., 2018) and with the white to gray matter ratio also similar to the human brain (Tallinen et al., 2014), pigs are often considered a better animal model than rodents in studying the human brain (Clouard et al., 2012). Morphometric analyses of the pig fetal brain by magnetic resonance imaging (MRI) have provided new insights into the cerebral white matter development (Qi et al., 2017), gestational influence on brain size (Conrad et al., 2012), and developmental 
abnormality and the application of prenatal diagnostics (Salomon and Garel, 2007). Longitudinal MRI studies have shown similarities in development between the pig and human fetal brain (Winter et al., 2011). The folding of the pig fetal brain follows similar patterns of neuronal networks and subcortical nuclei seen in humans and nonhuman primates (Larsen et al., 2004).

Molecular studies of the developing pig brain have also highlighted signatures in gene expression similar to that of the human brain (Sjöstedt et al., 2020). Multiple 5hydroxytryptamine (5HT) receptors, or serotonin receptors, including 5HT4, 5HT6, 5HT1D, and 5HT2C were expressed within the caudate nucleus of the pig brain that are highly homologous to 5HT1B in humans (Lind et al., 2007). Additionally, it is estimated that pigs and humans share a similar number of serotonin neurons within the raphe nucleus (Cumming et al., 2007). Pigs are also a great model for studying human sex differences in the brain development, particularly the hippocampal development that occurs 5 weeks sooner in females than males (Conrad et al., 2012). A dysfunction of maternal health during pregnancy, such as infection, is also known to impact gene expression of the fetal brain of pigs (Antonson et al., 2018). Recently, single-cell RNA sequencing performed in adult pig brains have provided new opportunities for a compendium and comparative epigenomics analysis between pigs and humans (Zhu et al., 2021). The previous study (Chapter 2) profiled gene expression of male and female fetal pig brains on gestation day (d) 45, 60 and 90, which identified genes differentially regulated between sex and gestation days.

It has been shown that regulation of gene expression in the pig brain is influenced by various epigenetic factors including chromatin modification and DNA methylation 
(Jarmasz et al., 2019). DNA methylation alters the epigenetic state of a genome, often referred to as an epigenome, during brain development in mammals (Lister et al., 2013). In the human fetal brain more than $7 \%$ of the genome is modified by methylation in a highly dynamic manner (Spiers et al., 2015). Reduced representation by bisulfite sequencing has shown that nearly $40 \%$ of $\mathrm{CpG}$ sites, compared to only $1.6 \%$ of non-CpG sites, are methylated in the 9-week-old pig brain (Choi et al., 2015). Recently, multitissue methylation analysis in pigs have identified sites associated with candidate epigenetic clocks that are relevant for aging and obesity in pigs (Schachtschneider et al., 2020). In addition, analysis of methylation of single nucleotide polymorphic (SNP) sites provides information about genetic-epigenetic interactions that modulate metabolic and regulatory processes in a tissue dependent manner (Schachtschneider et al., 2015). For example, DNA methylation accumulates in white blood cells with development and aging (Jia et al., 2019; Johnson et al., 2012; Merid et al., 2020; Terry et al., 2011), modulating the aging of different organs by altering the epigenetic clocks of individual organs (Simpkin et al., 2016). It is also known that specific methylation markers of blood are reliable biomarkers of neurodegeneration and neuropsychiatric diseases (Freytag et al., 2017; Lin et al., 2018; Walton et al., 2016). Research further shows there are correspondences in DNA methylation between the brain and peripheral blood in humans (Merid et al., 2020).

Despite the increase in the use of pigs in brain research, the understanding of DNA methylation of the pig brain, particularly at the fetal stages, remains obscure. The objectives of the present study were to 1) investigate changes in DNA methylation of the fetal brain as the brain develops from gestation day 45 to 60 and from day 60 to 90;2) 
establish the relationship between DNA methylation and gene expression of the fetal brain; 3) determine the influence of fetal sex on DNA methylation during brain development; 4) dissect genetic-epigenetic interaction in the fetal brain by analyzing methylation patterns of single nucleotide polymorphic sites; and 5) explore the correlation in DNA methylation of the fetal brain with that of adult blood.

\section{Materials and methods}

\section{Animals and experimental design}

In this study, all 18 fetal pigs and all 6 adult peripheral blood samples were obtained at the University of Missouri Swine Research Complex (Columbia, MO). The fetal tissues were dissected from sows bred via artificial insemination (AI). Dams were Landrace, Large White or a cross of these breeds and bred to Choice Genetics (Choice USA 1415 28th Street Suite 400 West Des Moines, Iowa 50266) LR-M6 line. Gestation day 1 (d1) was considered the day of AI. Dams (n=9) were euthanized at d45 (44-46), d60 (59-61) or d90 (89-91) via electrical stunning and exsanguination at the University of Missouri abattoir, a USDA inspected commercial unit (Establishment \#5077A).

\section{Fetal dissection}

The reproductive tract was removed from the dam at the abattoir. Individual fetuses, referred to as $\mathrm{d} 45, \mathrm{~d} 60, \mathrm{~d} 90$ male $(\mathrm{M})$ or female $(\mathrm{F})$ were removed from each uterine horn. One male and one female fetus was collected from each dam. No fetuses were taken from the tip of either horn or the body of the uterus. Each fetus was weighed before dissection. Additional information including litter size, and the sex of fetuses on 
the medial and lateral side of the fetus collected. The skin and skull were cut down the mid-sagittal plane with a scalpel and removed near the ears. The whole brain was scooped out, weighed, measured at the longest point, and stored on ice until homogenization. The fetal brain was homogenized with RLT buffer (Qiagen, Cat No./ID: 79216) with 2-mercaptoethanol using a benchtop VDI 25 tissue homogenizer (VWR). Aliquots of $1 \mathrm{ml}$ of the homogenate were stored at $-80^{\circ} \mathrm{C}$ for future analysis.

\section{DNA extraction from fetal brain}

Total DNA was isolated from frozen homogenized tissue samples using an AllPrep DNA/RNA Mini Kit (Qiagen, Cat No./ID: 80204) as per the manufacturer's instruction. The homogenate was briefly thawed and then centrifuged for 1 minute at $\geq 8000 \mathrm{x}$ g to separate debris. $750 \mu 1$ of the supernate was loaded to the spin column and centrifuged for 1 minute $\geq 8000 \mathrm{xg}$, placed in a clean collection tube, and centrifuged again for 1 minute $\geq 8000 \mathrm{x}$ g. DNA was eluted in $30 \mu \mathrm{l}$ elution buffer twice, incubating for 5 minutes each time. Thus, a total of $60 \mu 1$ DNA was eluted from each of the 18 samples (3 gestation days, 3 biological replicates, 2 fetal sexes) that were generated. DNA concentration was determined using a Qubit 2.0 Fluorometer (Life Technologies). Each sample was diluted to $100 \mathrm{ng} / \mu \mathrm{l}$ using nuclease-free water and equimolar amounts of DNA from the biological replicates of each sample were used to generate pooled DNA of the fetal brain. The pooled DNA fetal brain samples of both sexes at each gestational time point, resulting in a total of 6 samples, were separated in a $1.2 \%$ ethidium bromide-stained agarose gel and imaged on a ChemiDoc ${ }^{\mathrm{TM}}$ Touch Imaging System (Bio-Rad).

Blood collection 
Blood was collected from age-matched boars and sows. Each adult boar $(n=3)$ was age matched via farm records to a sow $(n=3)$, with each pair born within 1 calendar day of each other (Table 3.4). At the time of collection, the boar and sow pairs were 10 months (296/297 days), 12 months (364/365 days) and 21 months old (646/647 days). Each animal was restrained using a snare and whole blood was collected with a syringe and needle from the jugular vein and put in ethylenediaminetetraacetic acid (EDTA) tubes to prevent blood clotting. Blood tubes were kept on ice and transported to the lab.

\section{DNA extraction from blood}

Genomic DNA was isolated from fresh blood using a QIAamp DNA Blood Mini Kit (Qiagen, Cat No./ID: 51104) as per the manufacturer's instruction. DNA was eluted in $30 \mu l$ elution buffer twice, incubating for 5 minutes each time. Thus, a total of $60 \mu 1$ DNA was eluted from each of the 6 samples. Concentration and purity of the DNA was

determined using a Qubit 2.0 Fluorometer (Life Technologies). Each sample was diluted to $100 \mathrm{ng} / \mu \mathrm{l}$ using nuclease-free water. DNA $(2 \mu \mathrm{l})$ from each sample wan separated in a $1.2 \%$ ethidium bromide-stained agarose gel and imaged on a ChemiDoc ${ }^{\mathrm{TM}}$ Touch Imaging System (Bio-Rad).

Enzymatic methyl sequencing

DNA methylation profiling of all 12 samples (6 pooled fetal brain samples and 6 adult blood samples) was done by enzymatic methyl sequencing (EM-seq) using a NEBNext ${ }^{\circledR}$ Ultra $^{\mathrm{TM}}$ II DNA Library Prep Kit. The raw and processed data of DNA methylation are publicly available in GEO database (accession GSE178983). EM-seq 
utilizes the Ten-Eleven Translocation-2 (TET2) and oxidation enhancer to protect the methylated cytosines from deamination, whereas the unmethylated cytosines undergo deamination by APOBEC (apolipoprotein B mRNA editing enzyme, catalytic polypeptide). The resulting DNA from the two enzymatic processes (TET2 and APOBEC) have unmethylated cytosines, but the methylated cytosines get converted (deamination) to uracils. When those enzymatic treated DNA were used for PCR to make sequencing libraries, uracils were replaced by thymines, and $5 \mathrm{mC}$ and $5 \mathrm{hmC}$ were replaced by cytosines. Subsequently, when the libraries were sequenced, the unmethylated cytosines were represented by thymines, whereas the methylated cytosines were represented by cytosines. Then the reference genome sequences were converted (C/T and G/A), and the alignments reveal the methylated sites in the genome for the samples. All the 12 samples were enzymatically treated and processed to generate the libraries using the NEBNext ${ }^{\circledR}$ Ultra ${ }^{\mathrm{TM}}$ II DNA Library Prep Kit at the University of Missouri DNA Core Facility (Columbia, MO). The libraries were sequenced to 20x genome coverage using NovaSeq 6000.

Analysis of the enzymatic methyl sequencing data

The raw sequences were assessed for quality with FastQC. TrimGalore was used to remove adapters and perform base quality trimming. The reads obtained from the quality control steps were mapped to the reference genome of swine, Sscrofa11.1, using Bismark (Krueger and Andrews, 2011). After alignment, the methylation sites were extracted using the bismark_methylation_extractor algorithm implemented within Bismark to generate the coverage files for methylation sites. These files contain information for 
methylation sites including the chromosome, start position, end position, methylation percentage, count methylated, and count unmethylated data. The Bismark coverage files were then processed using edgeR (Chen et al., 2018) to filter sites that had at least 8 sequence reads, either methylated or unmethylated, in each sample. The methylated and unmethylated read counts were further filtered to remove sites that were always methylated or never methylated across the samples, as they provide no information about differential methylation. The methylated and unmethylated reads for a particular sample were treated as a unit, and hence were set to be equal in library size, which was the average of the total read counts for the methylated and unmethylated libraries.

Differential methylation analysis was then performed by fitting the normalized count data to a generalized linear model using the glmFit function.

\section{Statistical analyses}

All statistical analyses were performed using R. A likelihood ratio test was conducted using the $g \operatorname{lm} L R T$ function with the resulting model to identify the differentially methylated sites. The topTags function was used to extract the significant methylation sites with a false discovery rate $(\mathrm{FDR})<0.05$. The methylation sites were used to create circular Manhattan plot and SeqMonk visualization of methylation sites at the chromosome-level. The M-values (log ratio of methylated to unmethylated read counts) and FPKM expression values were used to calculate Euclidean distance between samples. The distance matrix was then used to perform hierarchical cluster analysis using the hclust base function in R. Mutual information network analysis was performed using the R package 'minet'. The canonical correlation of M-values between the fetal brain and adult blood were analyzed using cancor function in the R package CCA. To determine if 
changes in methylation between the male and female adult blood is correlated to methylation differences between the male and female fetal brain, the log fold changes of the differentially methylated sites (DMSs) in the blood of sows compared to boars (calculated by edgeR) was used with the M-values of the $\mathrm{CpG}$ sites to train an elastic net regularization model (Engebretsen and Bohlin, 2019). The trained model was selected based on ten iterations of alpha (mixing parameter) and lambda (shrinkage parameter) using the $\mathrm{R}$ package caret. The model was then used to predict the methylation changes in the fetal brain of males and females. The observed fold changes (calculated by edgeR), and the predicted fold changes (from elastic net regression) were compared to determine the correlation of methylation between the fetal brain and adult blood.

\section{Results}

DNA methylation of fetal brain at gestation day 45, 60 and 90

The data was pooled, regardless of sex, to examine the number of methylated sites at each time point. The fetal brain accumulated an increasing number of methylations as gestation day increased. A total of 583,843 CpG methylations were identified on $\mathrm{d} 45$, that increased to $1,232,170$ on d60 (Figure 3.1A) and 1,966,607 on d90 (Figure 3.1B). There were 90,441 methylation sites that were common between d45 and d60 and 166,803 commonly methylated sites bewteen d60 and 90, suggesting that the fetal brain accumulated an additional 1,141,729 methylations as it developed from day 45 to 60 (Figure 3.1A) and 1,799,804 methylations during development from day 60 to 90 (Figure 3.1B). Differential methylation analysis (Chen et al., 2018) showed changes in the methylation patterns between early ( $d 45$ to $\mathrm{d} 60$ ) (Figure 3.1C) and late gestation (d60 
to d90) (Figure 3.1D). This study also identified 95 sites that were hypermethylated (a higher level of methylation) and 50 sites that were hypomethylated (a lower level of methylation) on d60 compared to $\mathrm{d} 45$. On $\mathrm{d} 90$, relative to d60, only 21 sites were hypermethylated, but 42 sites were hypomethylated, suggesting that there was a significant change (Chi-square 18.44, $p<0.0001)$ in the methylation level of the fetal brain between $\mathrm{d} 45$ and 90 . The genes positionally closest to the sites that were methylated on each of the three gestation days were identified by BEDTools (Quinlan and Hall, 2010). Gene Ontology (GO) enrichment analysis (Ashburner et al., 2000) of the positionally closest genes that were common across the three days showed significant association with 396 biological processes. Of these 396 biological processes, embryonic hemopoiesis (GO:0035162) was the most enriched function. The PANTHER pathway enrichment analysis (Mi et al., 2013) consistently showed a significant enrichment (Fisher test, FDR < 0.04) related to the gonadotropin-releasing hormone $(\mathrm{GnRH})$ receptor pathway (P06664) at all three days.

The chromosomal position of methylated sites and their density (number of methylated sites per megabase of genome) remain highly conserved during development (Figure 3.2). However, there were certain genomic regions that were more prone to methylation during late gestation than early gestation. As shown in the density plots in Figure 3.2A, this research observed two hotspots of methylation, one in chromosome (Chr) 14 and the other in Chr 17 (both shown the red based on the color code scale in Figure 3.2A), in the fetal brain on $\mathrm{d} 45$ and 60 . But as the brain developed from day 60 to 90, additional methylation hotspots, one in $\mathrm{Chr} 5$ and the other in $\mathrm{Chr} 8$, were observed (shown in red in Figure 3.2B). In addition, Chr $\mathrm{X}$ accumulated a greater number of 
methylations during d60 to 90 as compared to d45 to 60. Chromosomes 1,11 and 18 remained largely cold for methylation in the fetal brain among the three gestation days. A significant bias $($ F-test $p$-value $=0.00018)$ was observed in the number of methylations in the $\mathrm{CpG}$ islands versus $\mathrm{CpG}$ shores in the pig genome (Choi et al., 2015). This study defines $\mathrm{CpG}$ shores as $2 \mathrm{~kb}$ sequences on either ends of the annotated $\mathrm{CpG}$ islands of the pig genome (Karolchik et al., 2004). More than a 3-fold increase in the number of methylations was consistently observed in the $\mathrm{CpG}$ shores than $\mathrm{CpG}$ islands at all the three gestation days.

\section{Relationship between DNA methylation and gene expression in the fetal brain}

The methylation level (M-value) was determined by the log ratio of the number of methylated to unmethylated reads in the male and female fetal brains on $\mathrm{d} 45,60$ and 90 . The Euclidean distance of M-values, by hierarchical cluster analysis, showed that the male and female fetal brains on d60 and 90 were relatively more similar in DNA methylation than those on $d 45$ (Figure 3.4A). The RNA-sequencing (RNA-seq) data generated from male and female fetal brains at the same gestation days (Chapter 2) was normalized to fragment per kilo base per million (FPKM) values. Hierarchical cluster analysis of the Euclidean distance of FPKM variation showed that gene expression in the male and female fetal brain is more similar between d45 and 60 compared to d90 (Figure 3.4B). Pair-wise correlation of Euclidean distance also agreed with the hierarchical cluster analysis (Figure 3.4C and 3.4D). These results suggested that DNA methylation and gene expression vary in an opposite pattern where variation in the DNA methylation decreases and variation in gene expression increases as the brain develops from d45 to 90 (Figure 3.4E). This global pattern in the variation in genome methylation and 
transcriptome of the pig fetal brain is consistent with results from the edgeR analysis that showed an increasing number of differentially expressed genes (DEGs) but decreasing number of differentially methylated DNA in the fetal brain between d60 and d90 compared to d45 and d60 (Table 3.1). However, DNA sites that were not detected as statistically significant in the edgeR analysis also showed variation patterns shown in Figure 3.4E. Thus, genome-scale epigenetic regulation, not just the $\mathrm{CpGs}$ detected from the edgeR analysis, are likely involved in the rapid increase in the fetal brain weight from approximately $1.3 \mathrm{~g}$ on d45 to nearly $19 \mathrm{~g}$ on d90 (Figure 3.4F).

\section{Methylation in the gene body and regulatory regions}

In this study, methylations in the gene body and in the immediate flanking DNA (within $1 \mathrm{~kb}$ ) of either end of the gene body were identified. Methylations that were specific to d45, 60 or 90, as well as those common between d45 and 60 as well as d60 and 90, were compared relative to their location to genes. The analysis showed DNA methylation accumulated in specific patterns relative to genes during brain development (Table 3.1). The data in Table 3.1 shows the number of methylation sites and their relative location to genes. The proportion of genes to methylation sites indicate the relative abundance of methylation in the upstream, downstream or gene body. During development from d45 to 60, a relatively higher proportion of methylation was found in the immediate downstream DNA (within $1 \mathrm{~kb}$ ) of genes than gene body and upstream sequences. Of all the methylations found common at $\mathrm{d} 45$ and 60, approximately $31.4 \%$ were found in the downstream compared to $23.7 \%$ found in the upstream DNA of genes. As the brain developed from d60 to d90, this pattern changed. Among the methylation sites that were commonly found on d60 and 90, a higher proportion of methylation 
occurred in the upstream than the downstream DNA of genes. On the other hand, the number of gene body methylations was low, in the range of 1 to $6 \%$ of the methylation at all three gestation times. The analysis also showed that introns were more prone (nearly 15-fold) to be methylated than exons at each time point (Table 3.2). The 5' and 3' untranslated regions (UTRs) were also differentially affected. The UTR-3' showed a higher proportion (average 3.7-fold) of methylation than the UTR-5'. Similarly, this study also observed a contrasting pattern of methylation between donor and acceptor splice sites of genes. The donor and acceptor sites are the 5' left and the 3' right ends of introns that are processed during alternative splicing of gene transcripts. Although the overall frequency of splice sites was low $(<1.5 \%$ of all methylations $)$, the proportion of donor splice sites was about $82 \%$ higher than that of the acceptor splice sites.

\section{Methylation of single nuclei polymorphic sites}

The data further showed that nearly one third of the methylation sites in the fetal brain were single nucleotide polymorphisms (SNPs). These variants were previously identified and obtained from Ensembl Sscrofa11.1 (Sus scrofa) variant database (Yates et al., 2020) and mapped to the $\mathrm{CpG}$ methylation sites to identify those that overlap in genomic position (referred to as CpG-SNP). A total of 212,487 CpG-SNPs were identified on $\mathrm{d} 45$, which accounted for $36.39 \%$ of all methylation sites detected at that time point. On d60, 33.89\% of the methylation sites were identified as CpG-SNPs $(\mathrm{n}=12,32,170)$ whereas on $\mathrm{d} 90$ 19,66,607 CpG-SNPs (accounting for $35.25 \%$ of all methylation sites) were identified. A majority of these methylations that occurred at the genetic variant sites were specific to each gestation time. Only 29,653 CpG-SNPs were common between d45 and d60, and 56,839 CpG-SNPs were common between d60 and 
d90, indicating a possible influence of gestation time on the epigenetic modification of DNA polymorphic sites during development of the fetal brain. Furthermore, nearly $62 \%$ of these methylated variant sites were localized within introns. Those in exons accounted for only 2.7\%. For all the methylation sites, irrespective of whether they were SNPs or not, similar differences between intron and exon sequences ( $~ 60 \%$ and $5.5 \%$, respectively) were found, suggesting that the higher abundance of CpG-SNPs in introns than exons is not due to methylation of the variant sites, but may be the intrinsic nature of the epigenome of the fetal brain.

\section{Sex differences in methylation of the fetal brain}

The data, which was pooled, regardless of gestation day, showed differences in the methylation landscapes between the male and female fetal brain. A total of 317,783 CpG sites were methylated in the brain of male fetuses during development as observed across the three gestation time points. A relatively larger number of sites $(n=406,397)$ were found methylated during the same developmental periods of the female brain. Only 65,147 of those were found common in the brain of both sexes, indicating that with pooled data across three gestation times, the methylation landscape of the fetal brain is largely distinct between males and females (Figure 3.3A). The common methylation sites $(\mathrm{n}=65,147)$ mostly showed a similar level of methylation in both sexes. However, differential methylation analysis identified 29 sites that were significantly different between males and females. Of these, 27 sites were hypermethylated in the male brain compared to the female brain. Only 2 were hypomethylated sites in the male brain compared to the female brain. The differential methylation in the fetal brain was highly biased in chromosomal location. The Chr X harbored the majority $(\mathrm{n}=21)$ of the 
differential methylation sites, and others were in chromosomes 3, 5, 6, 9,11 and 13

(Figure 3.3B). Each of these 21 sites in Chr X was hypomethylated in the male brain compared to the female brain. Besides Chr X, the cross-chromosomal methylation density plots show that the global methylation patterns were highly comparable (Figure 3.3C and 3.3D), indicating that $\mathrm{Chr} \mathrm{X}$ was likely the primary contributor of sex differences in methylation of the fetal brain.

\section{Canonical correlation in DNA methylation between the fetal brain and adult blood}

The methylation sites of blood collected from boars and sows were profiled and compared with the methylation sites of the fetal brain. The comparison showed 51,928 sites methylated in blood but not the brain, 27,040 sites methylated in the fetal brain but not in blood and 42,483 sites methylated in both the brain and blood. Interestingly, 5,492 of these methylation sites were known SNPs in the pig (known SNPs based on the Ensembl variation database) (Yates et al., 2020). Genes such as WRN, PEX5, APOE and $A R$ that were physically closest to $\mathrm{CpG}$ sites commonly methylated in fetal brain and adult brain were identified. These genes are related to aging in humans (Tacutu et al., 2018). The CpG sites of those genes were methylated in a mutually informative (Steuer et al., 2002) manner as evident from the network analysis (Figure 3.6). APOE and PEX5 genes were associated with downstream SNPs that also had mutually informative methylation patterns, indicating that genetic-epigenetic interactions are likely involved in the crosstalk between genes.

The common sites which were methylated between the fetal brain and adult blood, showed variable levels of methylation within and between the brain and blood samples. 
Cluster technique (Cheng and Church, 2000) was used to identify genomic sites whose methylation levels varied in a canonical correlation (CC) manner (Wilms and Croux, 2015) between the fetal brain and adult blood samples (Figure 3.5). This analysis identified distinct patterns of four groups of methylation sites. Groups 1, 3 and 4 showed negative correlation whereas group 2 showed a positive correlation in the methylation between the fetal brain and adult blood. Furthermore, 20bp sequences encompassing methylation sites canonically correlated between the fetal brain and adult blood were analyzed for motif enrichments using the HOMER (Hypergeometric Optimization of Motif EnRichment) suite of tools (Heinz et al., 2010). The analysis showed significant enrichment of binding motifs of specific transcription factors (Table 3.3), indicating that methylation may play a role in the recruitment of transcription factors (TF) to regulate dynamically changing chromatin of the fetal brain (de la Torre Ubieta et al., 2018; Gorkin et al., 2020; Héberlé and Bardet, 2019; Xuan Lin et al., 2019). An elastic net regularization modeling approach (Engebretsen and Bohlin, 2019) was applied to determine if changes in methylation between the male and female adult blood has concordance to the methylation differences between the male and female brain. A regression model was trained to learn the observed log fold changes in methylation differences in the adult blood, then that was used to predict the log fold change in methylation in the fetal brain. Comparison of observed and predicted values of methylation changes showed significant correlation (Spearman correlation 88.8, $\mathrm{p}=3.8$ e-28) (Figure 3.10), indicating that DNA methylation of blood and fetal brain have significant concordance. This finding is consistent with blood-brain methylation concordance in humans (Edgar et al., 2017). 


\section{Discussion}

This study investigated DNA methylation in the developing brain of pigs. There were $3,525,376$ methylations in total across the three gestation time points of the developing fetal brain. On average, 1,468 methylations per megabase $(\mathrm{MB})$ of the pig genome were observed. However, methylation in the fetal brain occurred in a non-random manner. Hotspots of methylation were observed in specific chromosomal regions (Figure 3.2). An earlier study with pig testis observed higher methylation variation in regions of low gene abundance (Wang and Kadarmideen, 2019), but this study found no significant differences in gene density and methylation in the fetal brain samples. Methylation occurs differentially in different tissues (Schachtschneider et al., 2020) which can explain differential chromosomal distribution in brain compared to other tissues.

GO analysis found significant association of specific developmentally related processes with the genes positionally closest to the methylation sites in the fetal brain. The previous study (Chapter 2) compared the gene expression profiles of the fetal brain with gene expression of the pig placenta (Wang et al. 2019) on d60 and d90. This comparative analysis identified 553 genes expressed in the fetal brain but not expressed in the placenta on d60. Another set of 87 genes showed an opposite pattern of expression at the same time point. A similar coordinated gene expression pattern between placenta and fetal brain was also observed on d90, suggesting a possible role of those genes in fetoplacental communication in the pig. The data also showed significant association of the gonadotropin-releasing hormone $(\mathrm{GnRH})$ receptor pathway in fetal brain development in pigs. In humans, GnRH-I and GnRH-II bind to GnRHR-I in the placenta to stimulate the production of the $\beta$-subunit of human chorionic gonadotropin, which is 
believed to influence maternal-fetal communication (Sasaki and Norwitz, 2011). DNA methylation in the placenta is a crucial determinant of fetal neurodevelopment (Jensen Peña et al., 2012; Laufer et al., 2021; Lester and Marsit, 2018). Regulation of the brainplacental axis is not only relevant to pregnancy outcomes (Behura et al., 2019a), but it can also influence the risk of diseases later in life (Bianco-Miotto et al., 2017). The development of the placenta and fetal brain is controlled by various epigenetic mechanisms (Cortes et al., 2019; Ji et al., 2017; Martin et al., 2017; Nelissen et al., 2011; Numata et al., 2012). Methylation at discrete sets of CpGs sites, often referred to as an 'epigenetic clock', have been used, not only to determine the biological age of organs, but also to predict the life span in mice and humans (Lee et al., 2019; Numata et al., 2012; Pavanello et al., 2020; Sierra et al., 2015; Stubbs et al., 2017; Tekola-Ayele et al., 2019). Studies show that the placental epigenetic clock is a reliable predictor of gestational age (Lee et al., 2019) and fetal growth (Bouchard, 2013; Ji et al., 2017; Nelissen et al., 2011). In the human placenta, DNA methylation occurs in a sex-biased manner to support fetal growth (Martin et al., 2017; Ratnu et al., 2017).

The analysis further showed contrasting patterns in gene expression and DNA methylation variation during development of the fetal brain. The variation in gene expression increased whereas variation of methylation decreased as the fetal brain developed from day 45 through day 90 (Figure 3.4). This observation is consistent with earlier findings that methylation and gene expression are inversely related, which is common in different tissues and species (Anastasiadi et al., 2018). The inverse relationship in gene expression and methylation in the pig fetal brain was further confirmed with the edgeR analysis that found a greater number of DEGs but a smaller 
number of DMSs between d60 and d90 as compared to d45 and d60. However, the genes that showed statistically significant differences in edgeR analysis were not the only contributor to the overall variability in gene expression in the fetal brain. Genes that were not statistically significant but showed marginal significance (FDR $<0.3)$ in the edgeR analysis were also found in the gene sets whose expression decreased with development of the fetal brain. Similar patterns in differential methylation analysis (Chen et al., 2018) were observed. The $\mathrm{CpG}$ sites, including both statistically significant (FDR $<0.05$ ), and non-significant (FDR < 0.25) sites, showed decreased methylation levels during brain development.

The role of methylation in X-chromosome inactivation (XIC) is a well-studied phenomenon (Cotton et al., 2015; Duncan et al., 2018; Sharp et al., 2011). XIC is required to silence majority of the genes of one $\mathrm{X}$ chromosome in female cells in order to achieve dosage compensation for genes between sexes. The $\mathrm{X}$-encoded genes, unlike genes on other chromosomes, are highly expressed in the brain (Nguyen and Disteche, 2006). Due to incomplete X inactivation in females, some genes escape XIC (Fang et al., 2019; Youness et al., 2021). Studies have shown that these XIC escapee genes tend to be highly expressed in the brain of females compared to males (Xu and Disteche, 2006). A study on the placenta and fetal brain of opossum suggested that DNA methylation plays a role controlling paternally imprinted XIC (Wang et al., 2014). Another study also showed that genes that escape XIC are associated with genetic variation of specific complex traits in humans (Sidorenko et al., 2019). The current study showed differences in DNA methylation of the X-chromosome of the fetal brain between males and females (Figure 3.3). Methylation in the X-chromosome occurred more abundantly in the brain of females 
than males. Methylation sites were mapped to known SNPs in the pig genome and identified disproportionately more sites of methylation in the genetic variants on the Xchromosome in the female fetal brain $(n=5,120)$ than the male fetal brain $(n=640)$. Such bias in DNA methylation of the $\mathrm{X}$-chromosome variant sites in the female versus male fetal brain indicate that both genetic and epigenetic changes contribute to gene expression differences in the brain of males and females (Ratnu et al., 2017). However, further studies are needed to establish if these changes are associated with genes that escape $\mathrm{X}$ inactivation during fetal brain development. Genes that escape $\mathrm{X}$ inactivation during fetal brain development is an important future direction as exposure of the fetuses to certain environmental chemicals, including bisphenol A (BPA) and diesel exhaust, is known to alter XIC processes in mice model studies (Kumamoto et al., 2013; Kumamoto and Oshio, 2013).

A key finding of this study is the canonical correlation in methylation specific sites between the fetal brain and adult blood (Figure 3.5). 1,475 sites were identified in the pig genome that were methylated in the fetal brain, irrespective of sex, during development. The same sites were methylated in a canonically correlated manner in the blood of adults, both in sows and boars. Blood DNA methylation is a reliable biomarker of development and aging (Hoshino et al., 2019). Although methylation occurs in a tissue-specific manner (Hoshino et al., 2019), there is concordance in methylation between the blood and brain during aging (Farré et al., 2015). Edgar et al. (2017) developed a web-based tool named 'BECon' (Blood-Brain Epigenetic Concordance) to search for methylation sites in blood that correlate with methylation in the human brain. The findings from the current study show that there were sites where there were concordances in methylation 
between the fetal brain and adult blood. As methylation in blood occurs in an age correlated manner (Farré et al., 2015; Horvath, 2013; Lee et al., 2019; Schachtschneider et al., 2020), this finding suggests that epigenetic changes that occur during development of the fetal brain are predictive of aging. This finding is consistent with the Dilman theory of developmental aging (DevAge) (Dilman, 1971), which suggests that aging and early development of the brain are regulated by common molecular processes. The DevAge theory has been supported by recent studies employing genomic and system biological approaches that establish functional connections between early life development and aging (de Magalhães and Church, 2005; Feltes et al., 2015). There is also emerging evidence that support the idea that aging and longevity programs originate early in life (Ackermann et al., 2007; Gluckman et al., 2008; Jagust, 2016; Pitale and Sahasrabuddhe, 2011). In the context of the DevAge theory, the findings from the current study have significant relevance to future research about the role of DNA methylation on the fetal origin of aging.

\section{Conclusion}

The current study evaluated DNA methylation changes between sexes during gestational development and biological aging between and within sexes. The data generated from this study will serve as a valuable resource for future investigation into epigenetic regulation of the fetal brain in pigs. It will also be helpful for dissecting sex biased gene regulation in the fetal brain and adult blood. 
Table 3.1. The number and location of methylations in the gene body and the immediate flanking DNA of genes on gestation day (d) 45, 60 and 90 in the fetal brain.

\begin{tabular}{|l|l|l|l|l|}
\hline Gestation day & $\begin{array}{l}\text { Methylation } \\
\text { location }\end{array}$ & $\begin{array}{l}\text { Number of } \\
\text { genes }\end{array}$ & $\begin{array}{l}\text { Number of } \\
\text { methylation sites }\end{array}$ & $\begin{array}{l}\text { Percentage (genes } \\
\text { relative to } \\
\text { methylation sites) }\end{array}$ \\
\hline $\mathrm{d} 45$ & 1kb_upstream & 1423 & 2896 & 49.14 \\
\hline $\mathrm{d} 45$ & Genebody & 12753 & 224527 & 5.68 \\
\hline $\mathrm{d} 45$ & 1kb_downstream & 1753 & 3254 & 53.87 \\
\hline $\mathrm{d} 45-\mathrm{d} 60$ & 1kb_upstream & 150 & 631 & 23.77 \\
\hline $\mathrm{d} 45-\mathrm{d} 60$ & Genebody & 5007 & 16133 & 31.04 \\
\hline $\mathrm{d} 45-\mathrm{d} 60$ & 1kb_downstream & 153 & 487 & 31.42 \\
\hline $\mathrm{d} 60$ & 1kb_upstream & 2878 & 6289 & 45.76 \\
\hline $\mathrm{d} 60$ & Genebody & 13828 & 496454 & 2.79 \\
\hline $\mathrm{d} 60$ & 1kb_downstream & 3279 & 7457 & 43.97 \\
\hline $\mathrm{d} 60$ - d90 & 1kb_upstream & 384 & 976 & 39.34 \\
\hline $\mathrm{d} 60$ - d90 & Genebody & 8624 & 46324 & 18.62 \\
\hline $\mathrm{d} 60-\mathrm{d} 90$ & 1kb_downstream & 432 & 927 & 46.6 \\
\hline $\mathrm{d} 90$ & 1kb_upstream & 4090 & 10808 & 37.84 \\
\hline $\mathrm{d} 90$ & Genebody & 14291 & 850419 & 1.68 \\
\hline $\mathrm{d} 90$ & 1kb_downstream & 4537 & 11901 & 38.12 \\
\hline
\end{tabular}


Table 3.2. The number of $\mathrm{CpG}$ methylated sites within exons, introns, splice sites and untranslated regions (UTR) of genes at gestation day (d) 45, 60 and 90 in the fetal brain. The values within parenthesis represent the percentage value of those sites relative to all the methylated sites observed during the specific gestation day(s).

\begin{tabular}{|l|l|l|l|l|l|}
\hline Location & $\mathbf{d 4 5}$ & $\mathbf{d 6 0}$ & $\mathbf{d 9 0}$ & $\mathbf{d 4 5}-\mathbf{d 6 0}$ & $\mathbf{d 6 0}-\mathbf{d 9 0}$ \\
\hline \multirow{2}{*}{ Exon } & 50,889 & 113,872 & 199,360 & 8,666 & 16,002 \\
\hline \multirow{2}{*}{ Intron } & $(4.318)$ & $(4.446)$ & $(4.581)$ & $(4.632)$ & $(4.434)$ \\
\hline \multirow{2}{*}{ Splice site acceptor } & 712,162 & $1,560,562$ & $2,648,965$ & 81,727 & 181,014 \\
& $(60.433)$ & $(60.926)$ & $(60.864)$ & $(43.682)$ & $(50.158)$ \\
\hline \multirow{2}{*}{ Splice site donor } & $(0.013)$ & $(0.012)$ & $(0.013)$ & $(0.015)$ & $(0.014)$ \\
\hline \multirow{2}{*}{ UTR-3' } & 13,562 & 31,692 & 55,981 & 1,654 & 3,284 \\
\hline \multirow{2}{*}{ UTR-5 } & $(1.151)$ & $(1.237)$ & $(1.286)$ & $(0.884)$ & $(0.91)$ \\
\hline & $(1,860$ & 31,202 & 54,068 & 1,635 & 3,561 \\
\hline
\end{tabular}


Table 3.3. Significant enrichment of binding motifs of specific transcription factors in sequences encompassing the methylation sites canonically correlated between the fetal brain and adult blood. Target incidence, background incidence, and log p-value are shown. Groups 1-4 are shown in Figure 3.5.

\begin{tabular}{|c|c|c|c|c|}
\hline $\begin{array}{l}\text { Methylation } \\
\text { group }\end{array}$ & Transcription Factor & $\begin{array}{l}\text { Target } \\
\text { incidence }\end{array}$ & $\begin{array}{l}\text { Backgroun } \\
\text { d incidence }\end{array}$ & $\log$ p-value \\
\hline group1 & OPI1 & $2.43 \%$ & $0.00 \%$ & $-4.09 \mathrm{E}+01$ \\
\hline group1 & RO3G_00049(RRM) & $2.12 \%$ & $0.06 \%$ & $-3.06 \mathrm{E}+01$ \\
\hline group1 & ANKHD1(KH) & $7.08 \%$ & $2.20 \%$ & $-2.85 \mathrm{E}+01$ \\
\hline group1 & TBS1 & $1.82 \%$ & $0.04 \%$ & $-2.79 \mathrm{E}+01$ \\
\hline group2 & Mad & $7.42 \%$ & $0.03 \%$ & $-3.25 \mathrm{E}+01$ \\
\hline group2 & STB4 & $14.45 \%$ & $2.13 \%$ & $-3.08 \mathrm{E}+01$ \\
\hline group3 & GATA8 & $13.73 \%$ & $0.42 \%$ & $-3.19 \mathrm{E}+01$ \\
\hline group4 & PB0179.1_Sp100_2 & $23.38 \%$ & $1.08 \%$ & $-2.32 \mathrm{E}+01$ \\
\hline
\end{tabular}


Table 3.4. Age matched adult blood between sows (S) and boars (B). The date of birth (DOB) and age at the time of collection are shown.

\begin{tabular}{|c|c|c|c|c|}
\hline Blood Sample & $\begin{array}{l}\text { Age } \\
\text { Matched }\end{array}$ & DOB & Age in months & Age in days \\
\hline Sow 1 (S1) & B1 & $10 / 26 / 2019$ & 10 months & 296 days \\
\hline Sow 2 (S2) & B3 & $8 / 19 / 2019$ & 12 months & 364 days \\
\hline Sow 3 (S3) & B2 & $11 / 9 / 2018$ & 21 months & 647 days \\
\hline Boar 1 (B1) & S1 & $10 / 25 / 2019$ & 10 months & 297 days \\
\hline Boar 2 (B2) & S3 & $11 / 10 / 2018$ & 21 months & 646 days \\
\hline Boar 3 (B3) & S2 & $8 / 18 / 2019$ & 12 months & 365 days \\
\hline
\end{tabular}


Figure 3.1. Venn diagrams showing the number of methylation sites between gestation day (d) 45 and 60 (A) and between d60 and d90 (B). Volcano plots showing hypomethylated and hypermethylated sites in the fetal brain on d60 compared to d45 (C) and d90 compared to d60 (D).

A

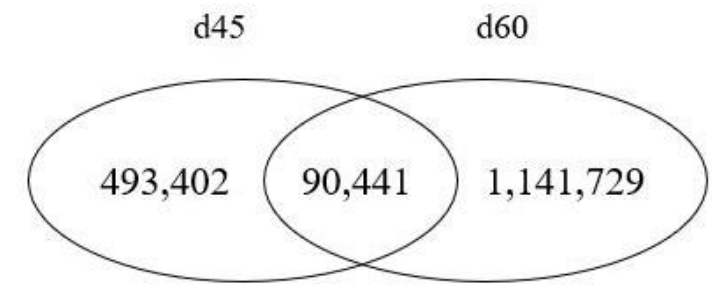

C

N

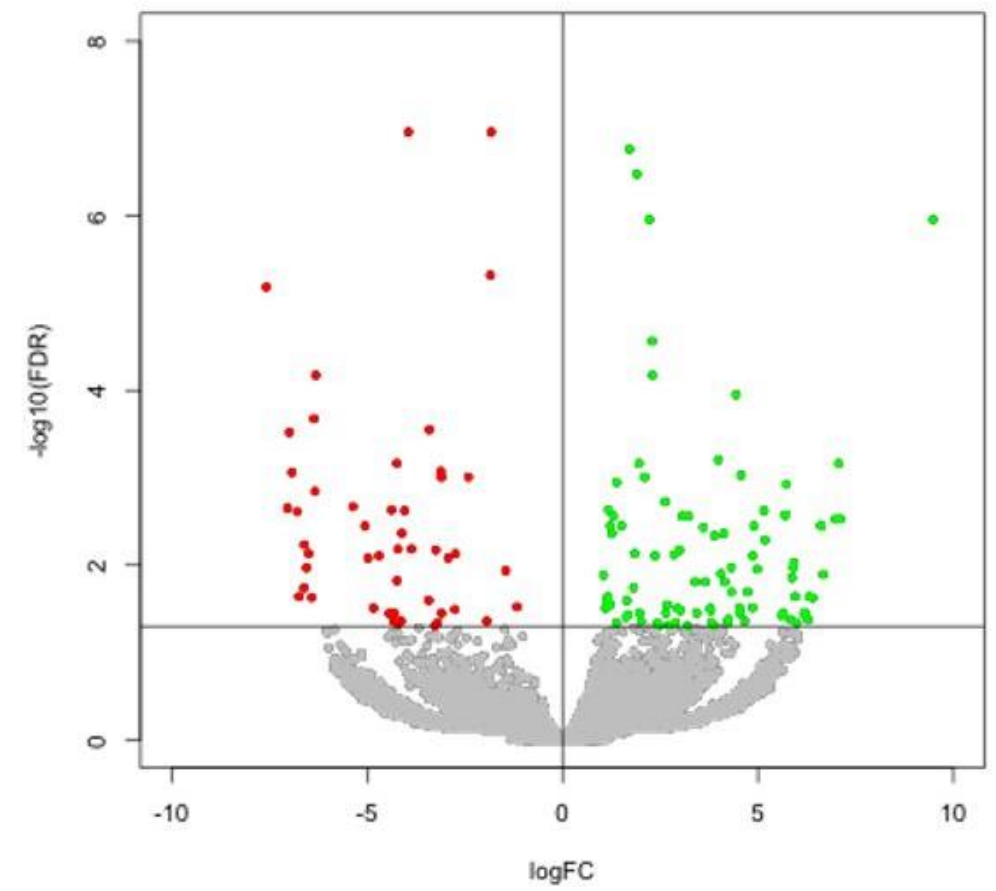

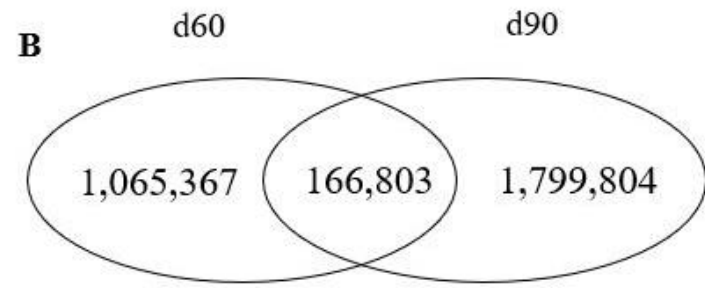

D

$\mathrm{d} 90-\mathrm{d} 60$

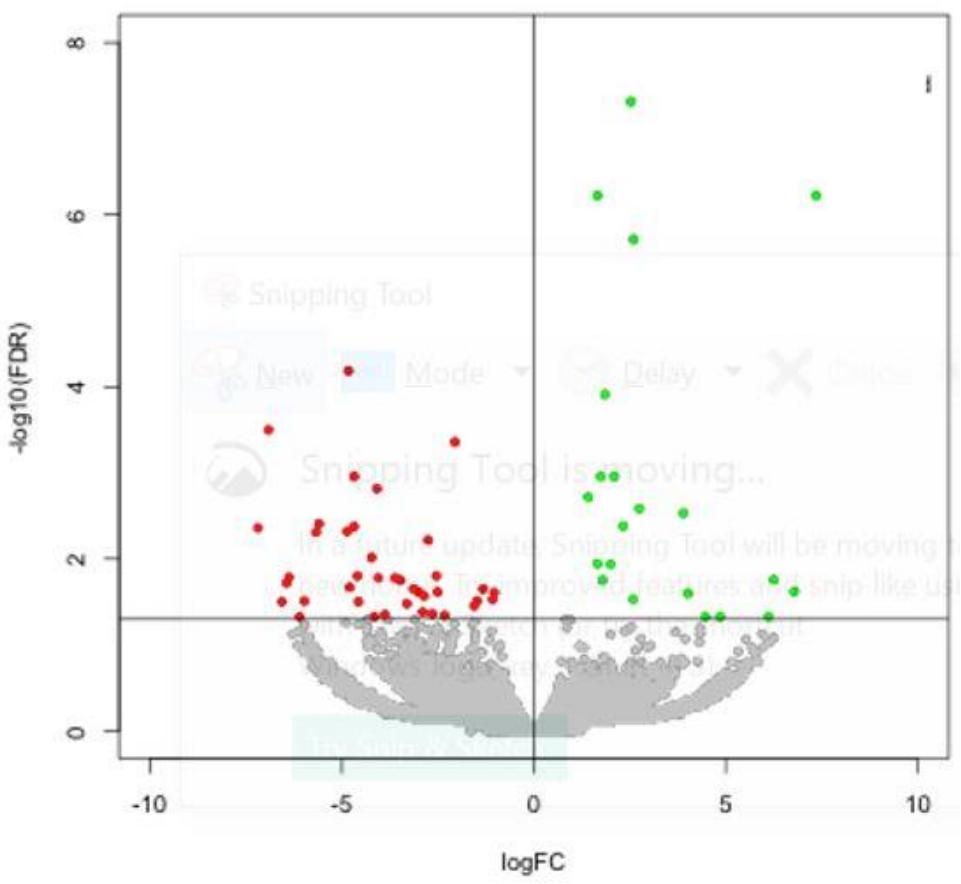


Figure 3.2. Chromosome-wise density plots of $\mathrm{CpG}$ methylation sites in the fetal brain that were common between gestation day (d) 45 and 60 (A) and d60 and d90 (B). The position scale is shown in intervals in 30 megabase (Mb) on the top and density scale is shown in color codes on the right. Common methylation across gestation day 45, 60 and 90 is shown in the SeqMonk local genome browser of Sscrofa11.1 (Sus scrofa) (C).

A Methylation density: gestation day 45-60

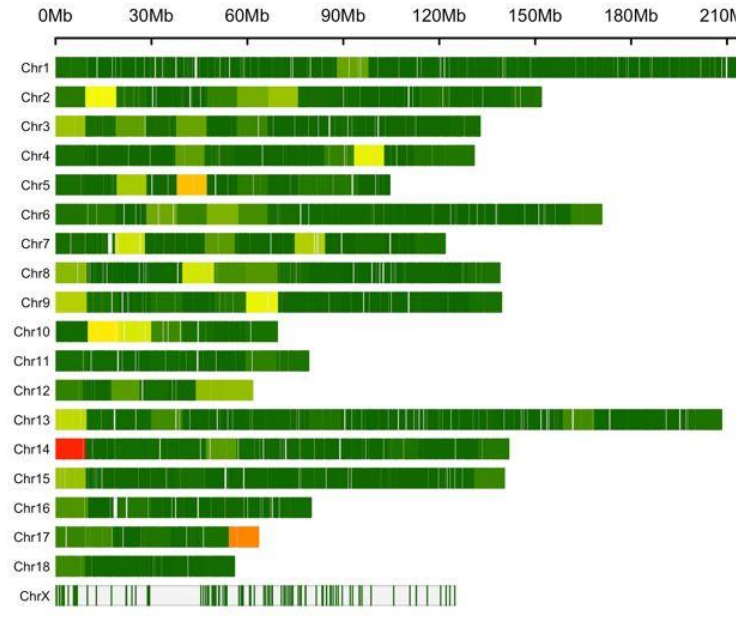

B

Methylation density: gestation day 60-90

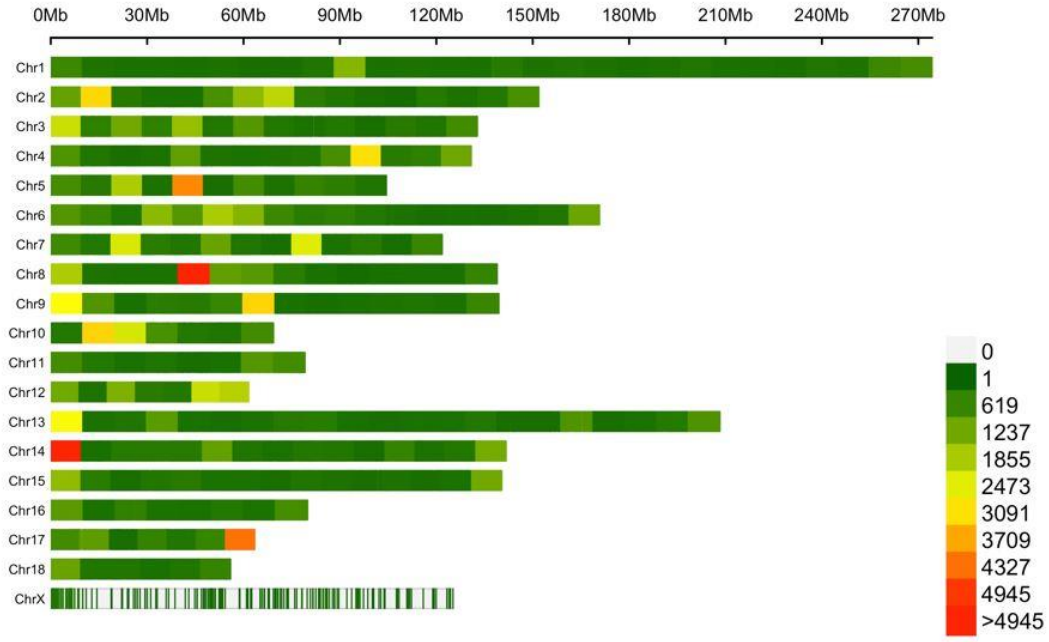

C

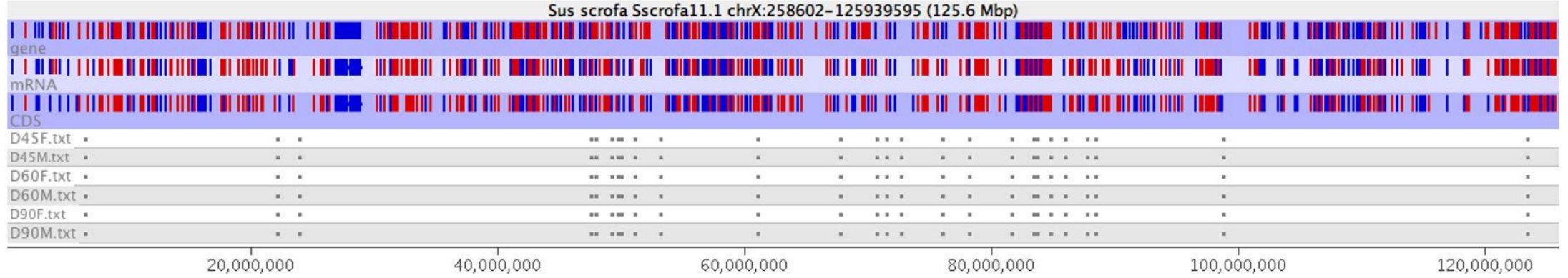


Figure 3.3. A. Venn diagrams showing the number of sex-specific methylation sites in the fetal brain. It also shows the sites that were commonly methylated in the fetal brain of both at gestation day 45, 60 and 90. B. Circular Manhattan plot of the - $\log 10 \mathrm{FDR}$ values of differentially methylated CpGs between the male and female fetal brain in each chromosome (Chr). C. Methylation density of the female fetal brain in each chromosome. D. Methylation density of the male fetal brain in each chromosome.

A Female fetal brain Male fetal brain

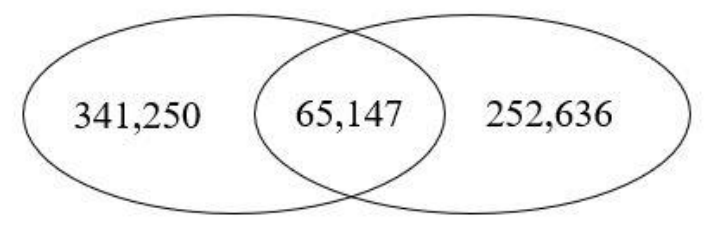

$\stackrel{+}{A}$

B

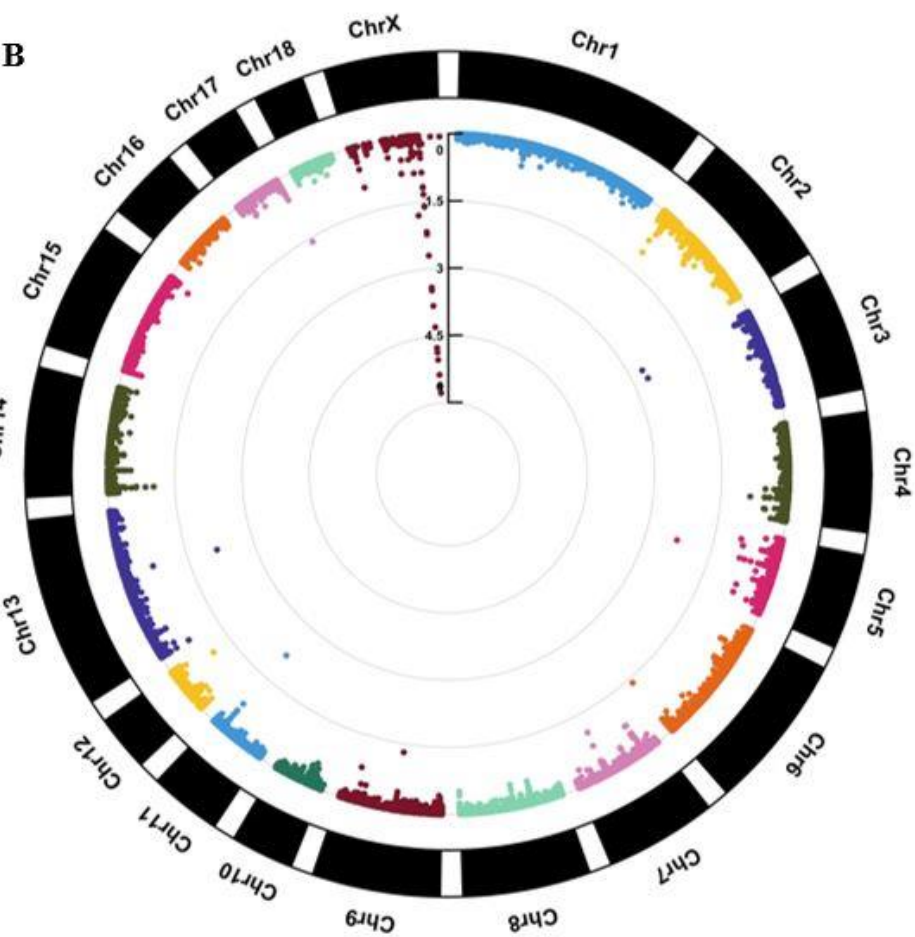

C Methylation density: female fetal brain

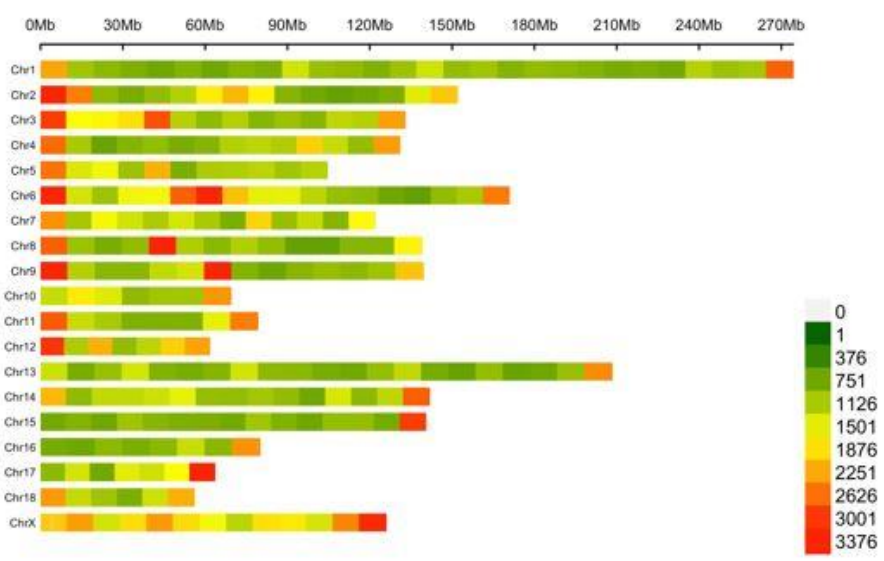

D

Methylation density: male fetal brain

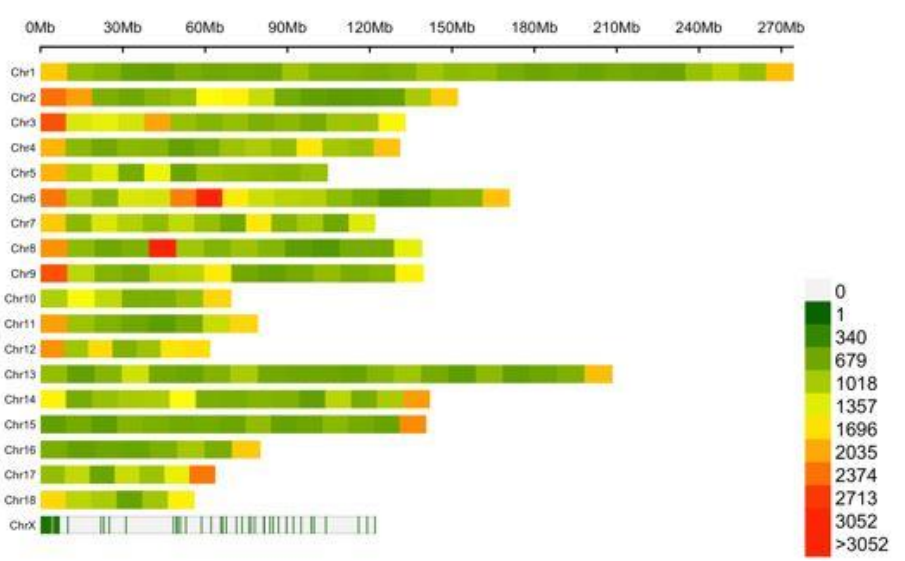


Figure 3.4. Hierarchical cluster analysis of DNA methylation $(\mathbf{A})$ and gene expression $(\mathbf{B})$ among the male $(\mathrm{M})$ and female $(\mathrm{F})$ fetal brain on gestation day (d) 45, 60 and 90. Euclidean distance of variation in M-values (log ratio of methylated to unmethylated read counts) was used in A, whereas that of FPKM values of gene expression was used in B. Pair-wise correlation in methylation $(\mathbf{C})$ and gene expression $(\mathbf{D})$ among male and female fetal brain samples at gestation day 45,60 and 90. E. A cartoon depicting the inverse relationship in variation of DNA methylation with gene expression during development of fetal brain from d45 to d90. F. Image and weight of pig fetal brain on d90 relative to d45.
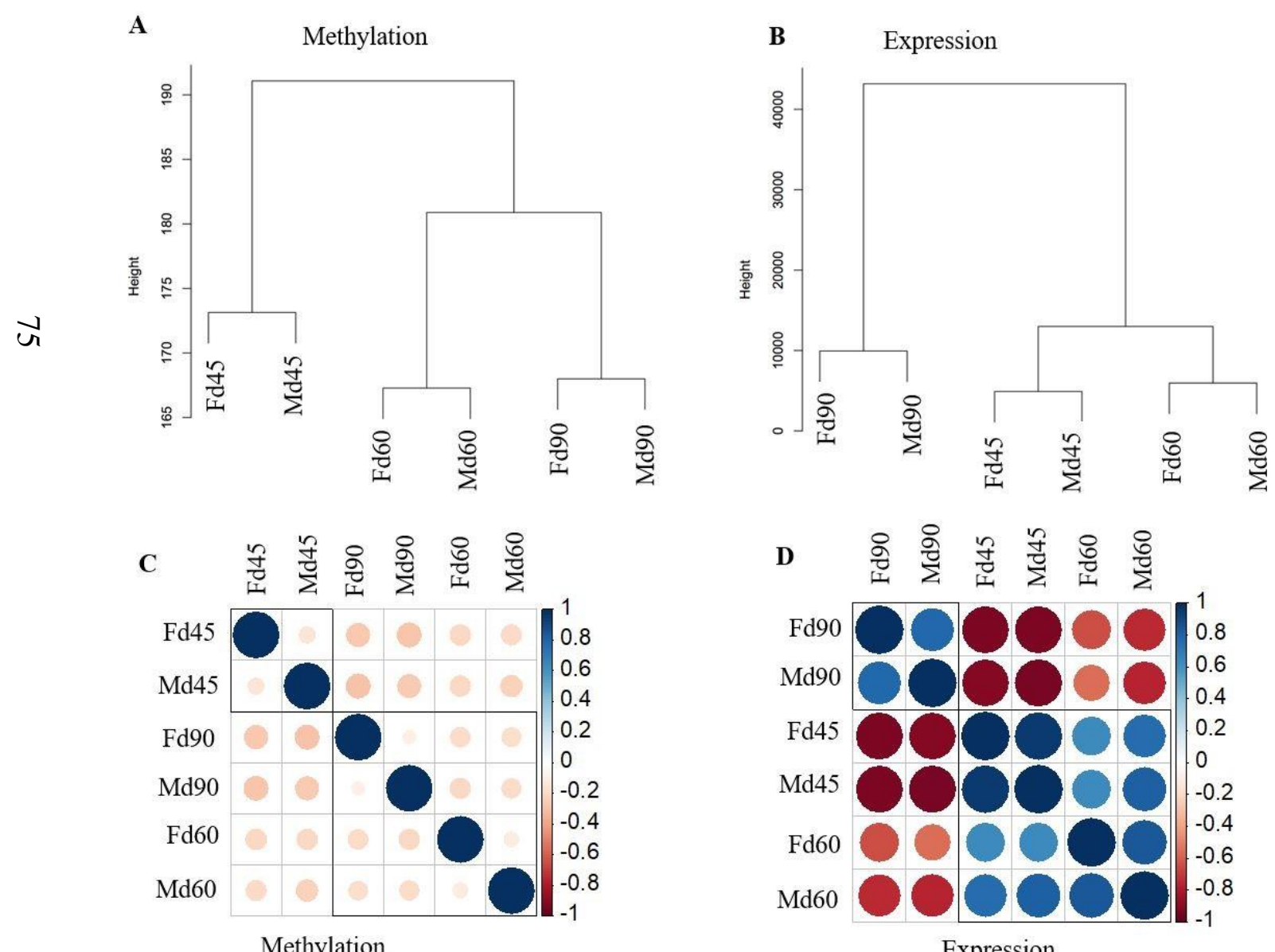

Expression

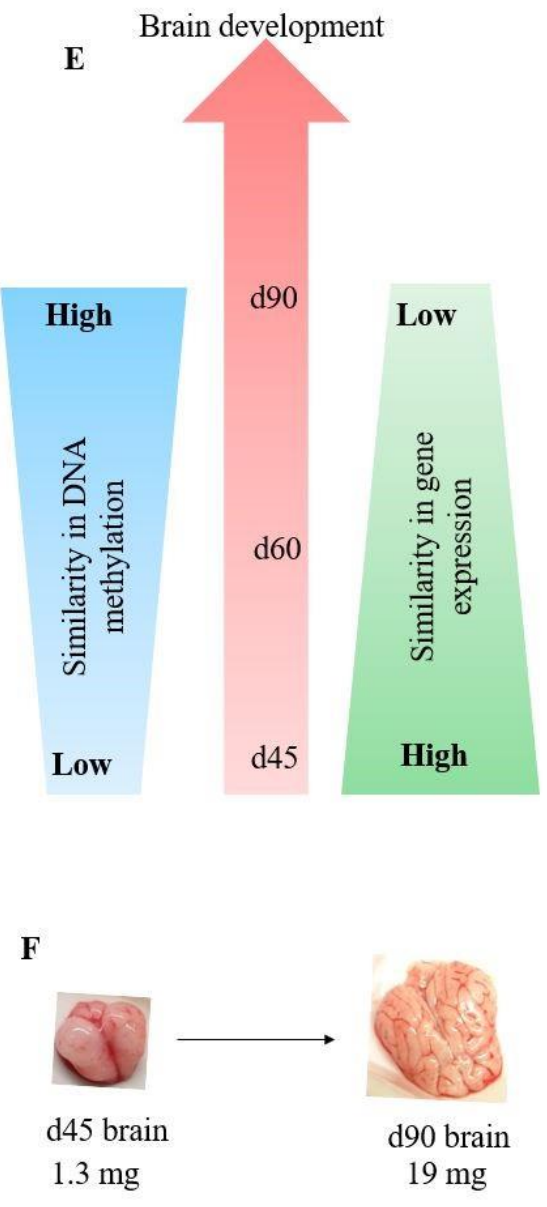


Figure 3.5. A boxplot showing four groups of $\mathrm{CpG}$ sites that were methylated in a canonical correlated manner between the fetal brain and adult blood samples. The x-axis shows the groups and y-axis shows the principal axis of variation in methylation level of the sites in the fetal brain. The horizontal line within each group (color coded) represents the mean value in correlation with whiskers within and outside the boxes for each group.

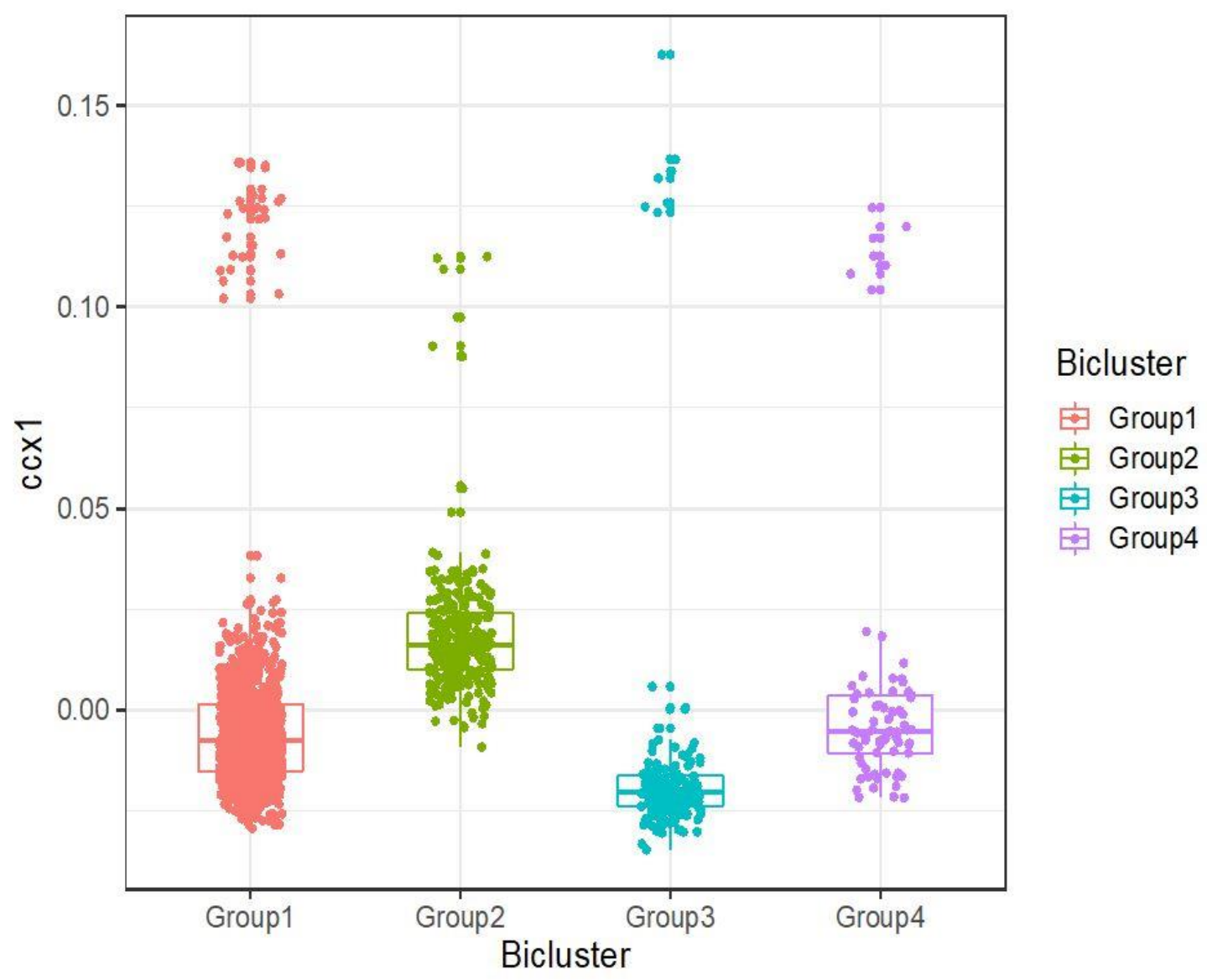


Figure 3.6. An arch plot showing a pair-wise mutual information network among methylation sites ( $\mathrm{n}=13$, shown as $\mathrm{m} 1 \mathrm{through} \mathrm{m} 13)$ to which APOE (Apolipoprotein E) is the closet gene. The methylation of known single nucleotide polymorphic sites are shown as ' $\mathrm{v}$ ' and the non-variant with are shown as 'nv'. The 13 sites are shown as black solid dots and the connecting arcs represent relationship (mutual information) among them.

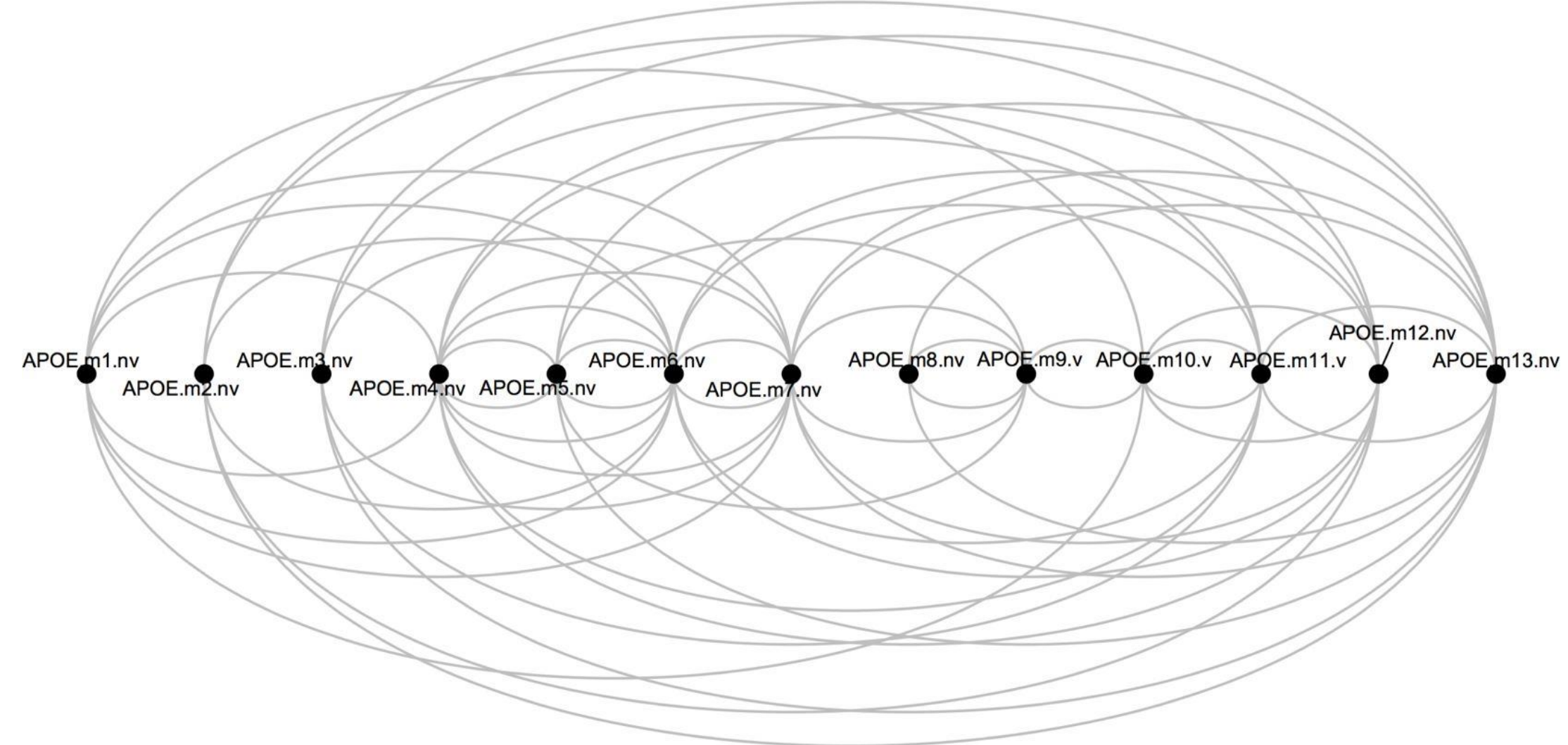


Figure 3.7. Mutual information network plots of methylation levels among sites in chromosome $X$ in male (A) and female (B) pigs across the fetal brain and adult blood samples. The methylation sites are shown as solid dots and the connecting curves (blue: males and orange: females) represent relationship among them.
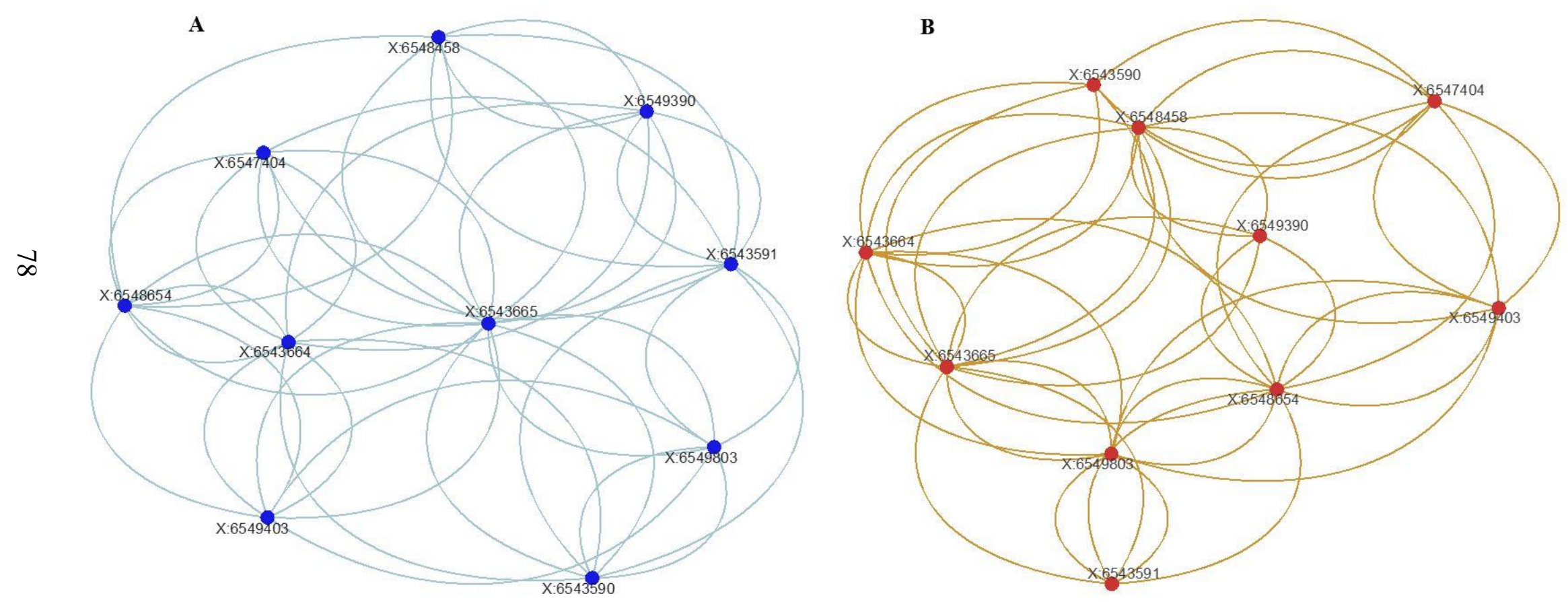
Figure 3.8. A. Venn diagram showing the number of sex-specific methylation sites in blood DNA of adult females and males. It also shows the sites that were commonly methylated in the blood of both sexes. B. A circular Manhattan plot of the $-\log 10 \mathrm{FDR}$ values of differentially methylated CpGs between the male and female adult blood DNA in each chromosome (Chr). C. Methylation density of the female adult blood in each chromosome. D. Methylation density of the male adult blood in each chromosome.

A

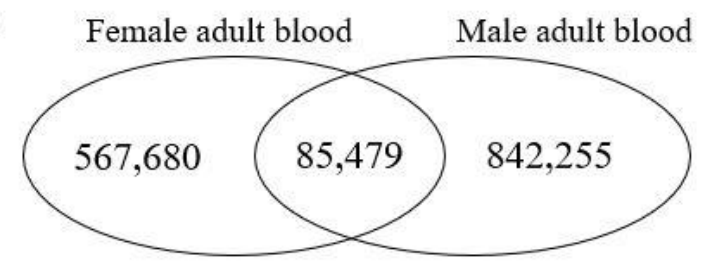

B

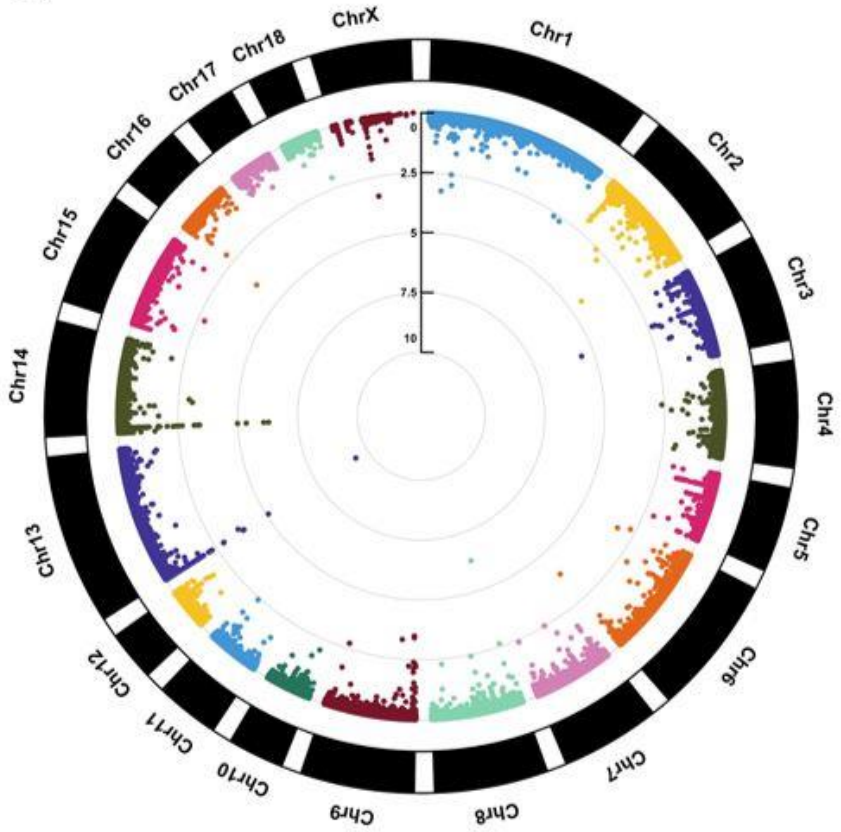

C Methylation density: female adult blood

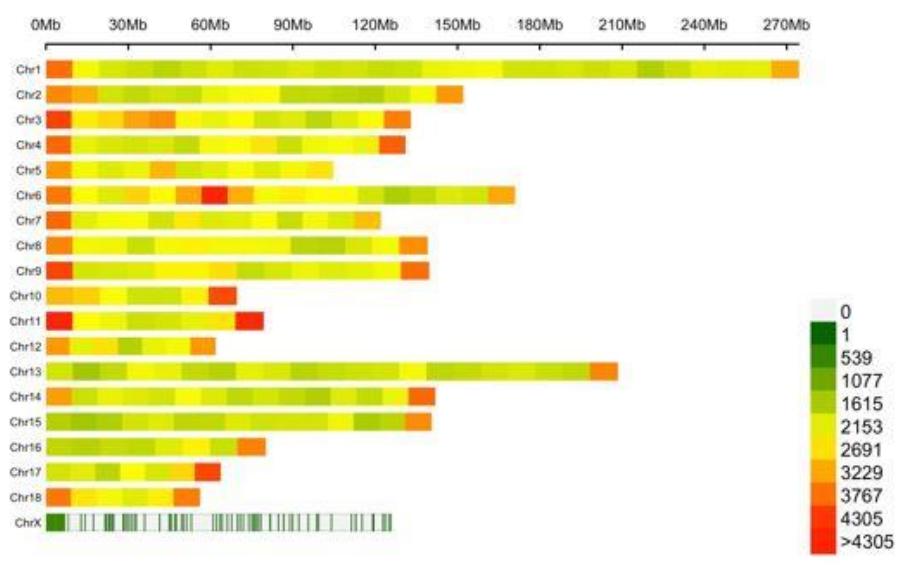

D Methylation density: male adult blood

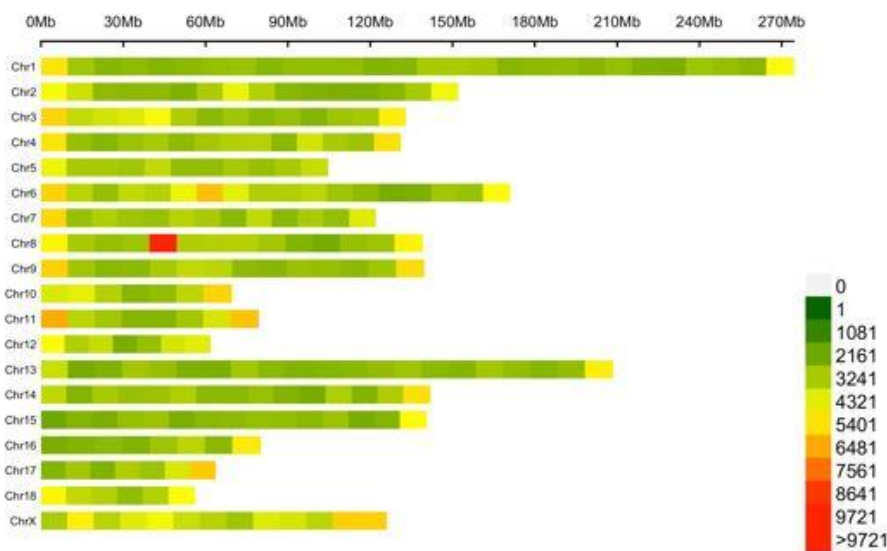


Figure 3.9. Principal component analysis of methylation sites in sows (S), boars (B) and the fetal brain at gestation day (D) 45, 60 and 90 that were associated with clusters (see Figure 3.5) (A) and those not associated with clusters (B).
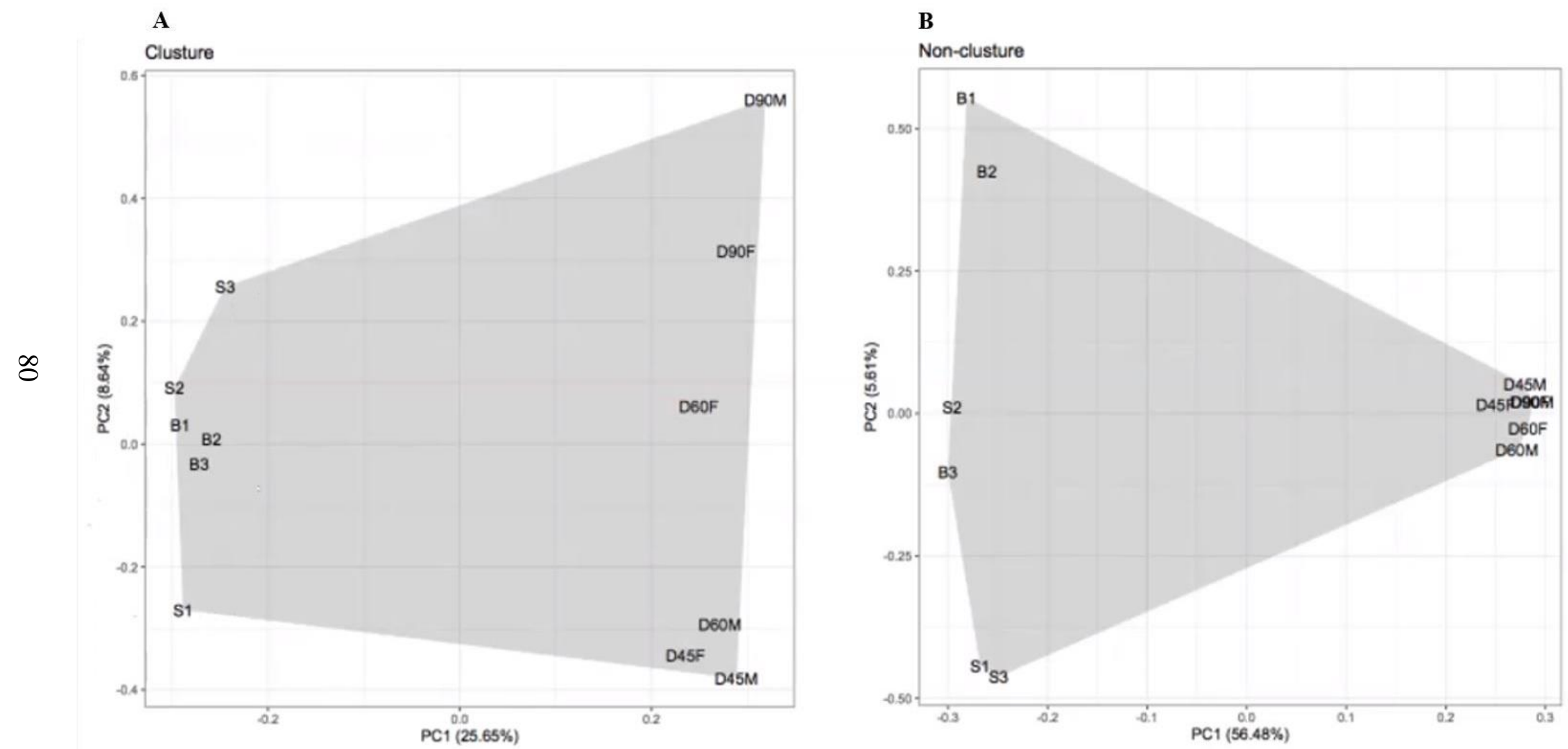
Figure 3.10. A plot showing the observed and predicted log fold change values of methylation sites showing canonical correlation in methylation between the fetal brain and adult blood.

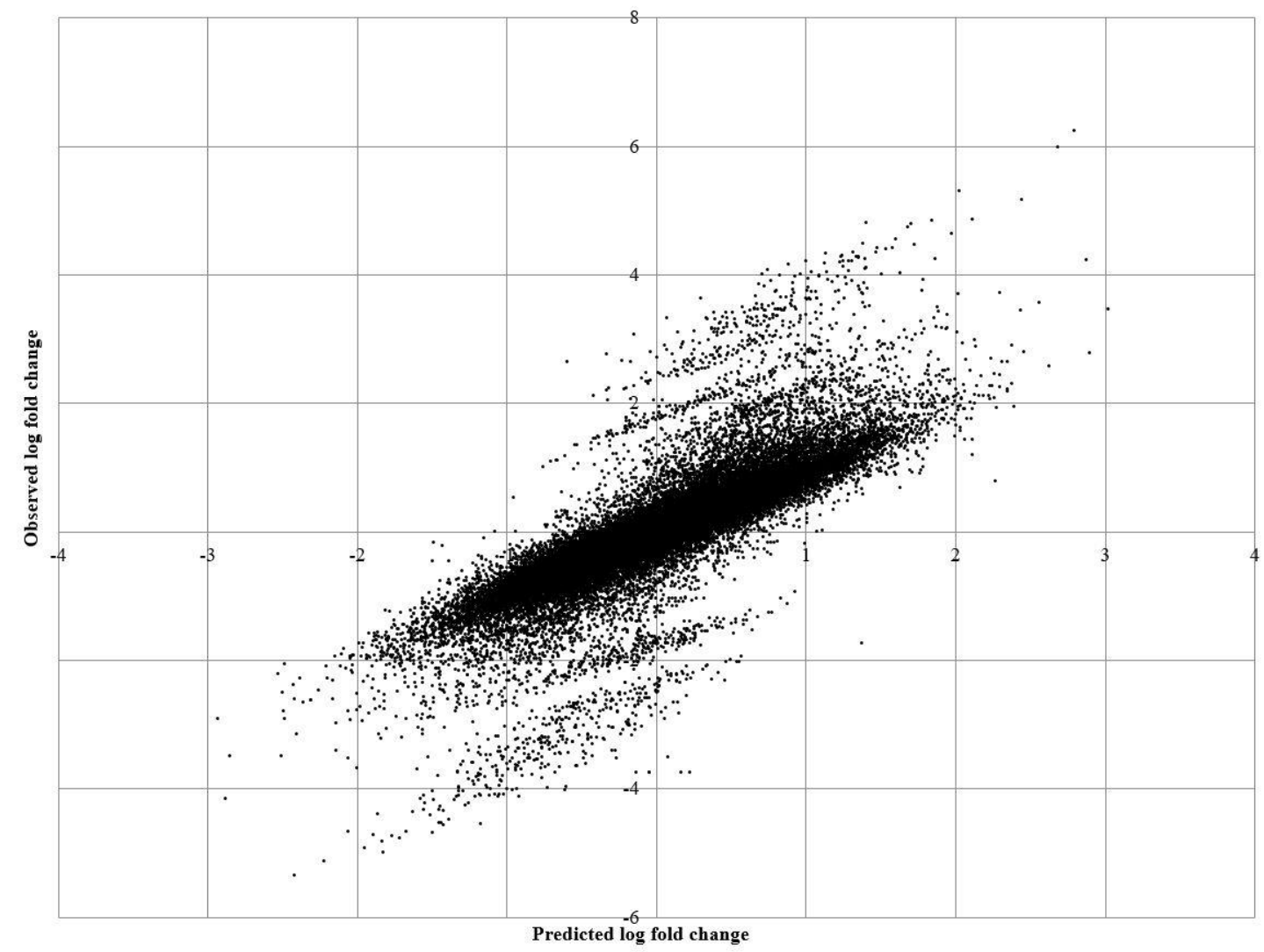




\title{
Chapter 4
}

\section{Comparative analysis of gene expression between the fetal brain and placenta of pigs and mice}

\author{
Abstract \\ This study compared the gene expression patterns of the fetal brain and placenta \\ between pigs and mice. The analysis identified 10 genes that were expressed in the \\ placenta of both pigs and mice. Each of these 10 genes (EPCAM, NRK, MBNL3, SPINT1, \\ ELF3, S100G, PRRG4, SMPDL3B, Clorf210, and TMPRSS2) were expressed (mean \\ fragment per kilobase million $(\mathrm{FPKM})>10)$ in the placenta but not expressed (mean \\ FPKM $<1$ ) in the fetal brain of both species. On the other hand, 112 genes that were \\ expressed in the fetal brain of both pigs and mice were identified. None of these genes \\ were expressed in the placenta of both species. Gene Ontology (GO) analysis of these \\ genes commonly expressed in the fetal brain of pigs and mice shows association with \\ biological functions of development of the head, central nervous system, neuronal \\ differentiation, regulation of neurotransmitter levels, neurogenesis, axonogenesis, and \\ cell-cell signaling. In-silico analysis of transcription factor binding sites in the $500 \mathrm{bp}$ of \\ the upstream DNA of these common genes, expressed either in the placenta or fetal brain \\ of both species, revealed that they were commonly regulated by the RE1 silencing \\ transcription factor (REST). REST is a multifaceted transcription factor that acts as a \\ master regulator of neurogenesis as well as controls the neural excitation and aging \\ processes. Although pigs and mice are phylogenetically distant species with different \\ forms of placentation, the similarity in the regulation of these genes between placenta and
}


fetal brain suggests an evolutionary conserved component of the brain-placental axis that may control brain development in placental species.

\section{Introduction}

Reproduction and development are highly diverse among the animal kingdom, even within the placental species (Ackerman, 1992). Placentae show differences in shape (i.e., diffuse, cotyledonary, zonary or discoid) and contact patterns of maternal tissue with the chorionic epithelium of the fetus (i.e., epitheliochorial, endotheliochorial or hemochorial). The pig placenta is diffuse and epitheliochorial (Leiser and Dantzer, 1988) whereas mice and human placentae are discoid and hemochorial (Soares et al., 2018). Despite these differences, basic placenta functions for fetal development remain highly conserved.

Previous research suggest that the placenta plays a key role in brain development (Zeltser and Leibel, 2011) and inadequate placental support can impact the developmental processes of the brain that increases the risk of brain diseases later in life (Behura et al., 2019a; Behura et al., 2019b; dos Reis et al., 2012; Zeltser and Leibel, 2011). There is a remarkable coordination in gene expression between the placenta and fetal brain of mice, suggesting robust regulation of the brain-placental axis (Behura et al., 2019a). The brain-placental axis also plays important roles in the fetal programming of brain development, and developmental origin of health and disease later in life in mice (Behura et al., 2019a). Similarities in placental function among species and the importance of the brain-placental axis led to the hypothesis that the placenta of pigs and mice have some commonalities in supporting fetal brain development. To test this 
hypothesis, this study performed cross-species comparative analyses of gene expression of the fetal brain and placenta between pigs and mice. The goal of this analysis was to identify genes that were regulated (express or not expressed) in the placenta but not the fetal brain (and vice versa) of both species.

\section{Materials and methods}

Gene expression data

The gene expression data from the fetal pig brain on gestation day (d) 60 and 90, regardless of sex, from a previous study (Chapter 2) was converted to fragments per kilobase million (FPKM) for comparison with gene expression data of pig placenta on d60 and d90, generated previously by Wang et al. (2019). The pig placenta gene expression data, in FPKM, was downloaded from the Gene Expression Omnibus (GEO) database (accession number GSE110414). The $\mathrm{d} 15$ mouse placenta and fetal brain gene expression data were previously generated in Susanta Behura's lab in a separate project (unpublished). The expression data are publicly available at GEO with this accession number (GSE157555).

\section{Identification of orthologous genes}

To compare the pig data to the mouse data, one-to-one orthologous genes between mouse and pig were downloaded from the Ensembl gene homolog dataset using biomart. A reciprocal orthologous mapping approach was applied to identify common genes (single-copy orthologs) between the pig and mouse. In this method, the single-copy orthologs of mouse genes in the pig genome were downloaded from the mouse Ensembl 
database (Yates et al., 2020). Similarly, the single-copy orthologs of pig genes in the mouse genome were downloaded from the pig Ensembl database (Yates et al., 2020). Both datasets were compared to find the common genes between the two species. The pig genes that had no ortholog in mouse and vice versa were also identified from the Ensembl homology database. The average gene expression of the pig fetal brain and placenta from d60 and d90 was used for comparison to the expression level of the singlecopy gene orthologs from d15 mouse fetal brain and placenta.

\section{Identification of genes expressed either in the fetal brain or placenta}

In this study genes were considered 'expressed' if the FPKM value was $>10$ and genes with FPKM $<1$ were considered 'not expressed'. This study did not further examine a gene with FPKM $\geq 1$ but $\leq 10$. These criteria were used to categorize genes that were either expressed in the placenta but not in the fetal brain, or expressed in the fetal brain but not in the placenta. Both the mouse and pig expression data were categorized the same way to compare expression of the orthologous genes.

Prediction of transcription factors that control gene sets in pigs and mice

Brain or placenta specific expression in both pigs and mice were analyzed for genes that are targets for any common transcription factor(s). The $500 \mathrm{bp}$ upstream region was searched for peak positions of 1120 chromatin immunoprecipitation sequencing (ChIPseq) tracks (ENCODE raw signals) and the rank order was determined to predict the cognate transcription factor. This analysis was performed using iRegulon method (Janky et al. 2014) implemented in the Cytoscope tool. The iRegulon is a gene set analysis 
method to perform in silico identification of regulatory factors of genes by mapping incidences of transcription factors binding motifs in the upstream DNA.

\section{Results}

Identification of genes that show an 'ON/ OFF' expression pattern in the fetal brain or placenta of pigs

On d60, 553 genes were expressed in the fetal brain but not expressed in the placenta, and 87 genes were expressed in the placenta but not in the brain were identified. On d90, a total of 553 genes were expressed in the fetal brain but not in the placenta, and 80 genes were expressed in the placenta but not in the brain (Table 4.1). Between d60 and d90 429 common genes were identified in the fetal brain (Figure 4.1A). Between d60 and d90 the placenta showed 60 common genes (Figure 4.1B).

Orthologous genes in pigs and mice

By applying a reciprocal orthologous mapping strategy (see Methods), a total of 15,376 one-to-one orthologous genes between pigs and mice were identified (Table 4.3). The one-to-one orthologous genes are single-copy genes that are common in pigs and mice. There are also other types of homologies such as many-to-one, one-to-many and many-to-many between pig and mouse genes, but this study excluded those genes in this analysis. In addition, this study identified 3,666 genes present in pigs but absent in mice and 3,569 genes present in mice but absent in pigs.

Gene expression in pigs compared to mice 
Pig genes that showed an $\mathrm{ON} / \mathrm{OFF}$ expression pattern were compared between the fetal brain and placenta with one-to-one orthologous genes in mice that also showed an ON/ OFF expression pattern between the fetal brain and placenta. The mouse expression data were downloaded from GEO (accession number GSE157555). The read count data were converted to FPKM values, and genes specific to either the placenta or fetal brain were identified by applying the criteria described previously. Then the genes that showed one-to-one orthologs with pig genes were identified. Using this approach, 414 genes that were expressed in the pig fetal brain at d60 and d90 and were one-to-one orthologs between mice and pigs were identified. Also, 50 common genes that were expressed in the pig placenta at d60 and d90 and were 1:1 orthologous gene with mice (Figure 4.3) were observed. At d60, this study revealed 171 pig genes that were expressed in the mouse fetal brain but not the placenta and 92 genes that showed the opposite pattern. Whereas, at d90, 553 pig genes were expressed in the mouse fetal brain but not the placenta and 80 pig genes were expresses in the mouse placenta but not the fetal brain (Table 4.2). When comparing the fetal brain specific genes in both species 112 common genes were identified (Figure 4.2A). Gene Ontology (GO) analysis showed that these genes are largely associated with neurogenesis and brain development, and the regulation processes. Comparing the placenta specific genes in both the species, 10 common genes were identified. Those genes were EPCAM, NRK, MBNL3, SPINT1, ELF3, S100G, PRRG4, SMPDL3B, C1orf210, and TMPRSS2 (Figure 4.2B).

Prediction of transcription factors that target the common genes 
The 112 fetal brain genes and the 10 placenta genes from both species were used to analyze the transcription factor binding sites within 500 bp of the upstream DNA using iregulon. The results of this analysis showed that REST binding sites are enriched in the upstream DNA of different subsets of these common genes. The minimal NES (normalized enrichment score) varied from 3.1 to 5.8, which was within the accepted range to validate the prediction (Janky et al. 2014). The AUC values (area under curve) ranged from 0.08 to 0.14 as the threshold prediction, which was within the range to accept the prediction (Janky et al. 2014).

\section{Discussion}

This study was carried out to perform a cross-species comparative analysis of gene expression regulation between the fetal brain and placenta in pigs and mice. A total of 553 genes were expressed in the pig fetal brain but not in the placenta on d60. On d90, 554 genes were expressed in the fetal brain but not in the placenta (Table 4.1). 429 of these were common genes expressed in the fetal brain of pigs on d60 as well as d90 (Figure 4.1A). One of those common genes is the sperm-tail PG-rich repeat containing 4 (STPG4) gene which is a maternal factor that plays a role in epigenetic chromatin reprogramming during early development of the zygote and is involved in the regulation of gametic DNA demethylation (The UniProt Consortium, 2019). This study identified 87 genes that were expressed in the placenta but not in the brain of pigs at d60 and 80 genes at d90 (Table 4.1). 60 of these genes were common between both gestation days, including the PAGE Family Member 4 (PAGE4) gene (Figure 4.1B). PAGE4 protects cells from stress-induced apoptosis by inhibiting reactive oxygen species production and 
via regulation of the mitogen-activated protein kinase (MAPK) signaling pathway (The UniProt Consortium, 2019). The MAPK pathway plays a critical role in the regulation of cell proliferation and communication of an extracellular signal to the nucleus (Zhang and Liu, 2002).

112 genes were expressed in the fetal brain of pigs and mice but absent in the placenta of both species. Included in the common genes is Dorsal Inhibitory Axon Guidance Protein (DRAXIN) and Neural EGFL Like 2 (NELL2) (Figure 4.2A). DRAXIN, in mice is required for the development of the spinal cord and forebrain commissures and acts as a chemorepulsive guidance protein for commissural axons during development (The UniProt Consortium, 2019). NELL2 is involved in the regulation of hypothalamic GNRH secretion and the control of puberty (The UniProt Consortium, 2019). The 112 common genes were analyzed using PANTHER Pathway, this analysis found that these genes were involved in development of the head, CNS, and neurons as well as neuronal differentiation, regulation of neurotransmitter levels, neurogenesis, axonogenesis, and cell-to-cell signaling.

10 common genes (EPCAM, NRK, MBNL3, SPINT1, ELF3, S100G, PRRG4, SMPDL3B, C1orf210, and TMPRSS2) were expressed both in the pig and mouse placenta but absent in the fetal brain of both species (Figure 4.2B). Although these animals have different types of placentation, this study identified common gene expression in the placenta that is vital for fetal viability. The epithelial cell adhesion molecule (EPCAM) gene may provide an immunological barrier against mucosal infection, and it may play a role in embryonic stem cell proliferation and differentiation (The UniProt Consortium, 2019). The Nik related kinase (NRK) gene is highly expressed in the placenta, regulates 
cell proliferation and development of placental tissues (Denda et al., 2011). The Muscleblind Like Splicing Regulator 3 (MBNL3) gene is abundant in the placenta of humans and has low expression in muscle. The $M B N L 3$ gene inhibits muscle differentiation by altering the splicing pattern of pre-mRNA (Lee et al., 2010). The Serine Peptidase Inhibitor, Kunitz Type 1 (SPINT1) gene displays high cell type-specific expression in the villous cytotrophoblasts of human placenta and in basal chorionic trophoblast cells of mice, and regulates placental labyrinth formation (Walentin et al., 2015). It is also known to be an essential regulator of mouse placentation with Spint1-/mice displaying an early placenta defect with lethality at embryonic day 10.5 (Walentin et al., 2015). In humans and mice, the E74 Like ETS Transcription Factor 3 (ELF3) gene may play an important role in epithelial cell differentiation, be associated with mammary gland development and involution, and play an important role in the regulation of transcription with TATA-less promoters in preimplantation embryos, which is essential in preimplantation development (The UniProt Consortium, 2019). The S100 calciumbinding protein $G(S 100 G)$ is expressed in the uterine endometrium during the estrous cycle and pregnancy of mice, pigs, and rats. $S 100 G$ is also regulated by estrogen and has been suggested to play an important role in the establishment of pregnancy in pigs (Choi et al., 2012). The molecular function of the proline rich and gla domain 4 (PRRG4) gene is not fully understood, but $P R R G$ proteins are highly expressed in the spinal cord, and PRRG4 protein has been found in the Golgi apparatus (Justice et al., 2017). PRRG4 may be a functional homolog of Drosophila commissureless, which is required for axon guidance and synapse formation in the fruit fly (Justice et al., 2017). The Sphingomyelin Phosphodiesterase Acid Like 3B (SMPDL3B) gene has in vitro phosphodiesterase activity 
in humans, but the physiological effects are poorly understood (The UniProt Consortium, 2019). The Chromosome 1 Open Reading Frame 210 (C1orf210) gene may be involved in membrane trafficking between endosomes and plasma membrane in humans (The UniProt Consortium, 2019). While the biological functions of the mammalian Transmembrane protease serine 2 (TMPRSS2) gene has not been determined, other serine proteases are known to play essential roles in many processes including coagulation, digestion, hormone processing, wound healing, embryonic development, immune responses, and cancer progression (Kim et al., 2006). TMPRSS2 has been shown to be expressed in the epithelium of the human placenta and is indispensable for cell entry of severe acute respiratory syndrome coronavirus 2 (SARS-CoV-2) (Li et al., 2020; Pique-Regi et al., 2020). While the function of some of these genes is not fully understood in pigs and mice, this study identified common gene expression between pigs and mice that have different anatomical placental types. Comparative genomics have also shown that orthologs of over $80 \%$ of the genes known to be required for proper placental development in mice are also expressed in the human placenta (Cox et al., 2009). Mouse and human placenta are more anatomically similar than mouse and pig placenta, but the common genes between the two show that there are functions of genes that are conserved among species and imperative for normal placental development in mammals.

The study further showed that REST is the common regulator of the genes expressed either in the placenta or the fetal brain of both pigs and mice. REST is a multifaceted transcription factor (Ooi and Wood, 2007) and plays a role in controlling other biological processes including embryonic stem cell self-renewal, cellular plasticity, neuronal excitement, and longevity (Gopalakrishnan, 2009; Zullo et al., 2019). In the embryonic 
stage, REST promotes stem cell pluripotency by suppressing neuronal genes in embryonic stem cells and blocking neuronal differentiation (Gupta et al., 2009; Singh et al., 2008). A recent study has shown that REST controls processes of neuronal activities and acts as a potent regulator of longevity (Zullo et al., 2019). Studies have shown that REST is expressed in the placenta, although the precise functions are unknown (Oda et al., 2004; Starks et al., 2019). An earlier study showed that genes suppressed in the placenta relative to the fetal brain of mice were significantly enriched with binding motifs of REST (Behura et al., 2019b). In the immediate upstream DNA (within $500 \mathrm{bp)} \mathrm{of} \mathrm{these}$ genes, the known REST binding motifs were over-represented, based on iRegulon analysis (Janky et al., 2014).

In a separate study, Susanta Behura's lab has identified REST target genes $(\mathrm{n}=641)$ in the placenta of male and female fetal mice on $\mathrm{d} 15$ by ChIP-seq. The study has not yet been published, but the ChIP-seq data are available at GEO under the accession number GSE157552. While most REST peaks were identified in the placenta of both sexes, nearly 17 -fold more peaks were observed in the placenta of male fetuses compared to the placenta of female fetuses. Motif enrichment analysis showed that both canonical and non-canonical RE1 motifs were recognized by REST. The analysis also showed sexspecific enrichment of REST binding sequences in the d15 placentae. Nearly $74 \%$ of peaks were localized within gene promoter regions. GO analysis of REST target genes associated with placenta development and functions, such as Cited2, Ccnf, Aridla, Krt8 and $C d k n 1 b$ (Jaquemar et al., 2003; Wang et al., 2016; Withington et al., 2006) were targeted by REST in the male placenta only. On the other hand, genes associated with chromatin modification, protein modification processes, and protein metabolic processes 
were targeted by REST in the female placenta only. The genes commonly targeted by REST in placentae of male and female fetuses were related to different regulatory processes, including regulation of protein depolymerization, microtubule polymerization, protein-containing complex disassembly, and keratinocyte proliferation. Given these findings in mice research, it remains to be seen how REST regulates target genes in the pig fetal brain and placenta.

\section{Conclusion}

The goal of this study was to investigate the similarities between gene expression in the fetal brain and placenta of pigs and mice to further understand evolutionary conserved genes during development, and the role of gene expression in the brain-placental axis. This goal was accomplished using gene expression data from fetal pigs and mice to perform comparative analyses of gene expression between the species. Although mice and pigs are phylogenetically distant species, and they have different means of reproduction and types of placentation, this study identified conserved genes and common gene expression during development in the fetal brain and the placenta. In particular, understanding of the role of REST in the brain-placental axis in pigs will provide valuable information to develop pigs as a model to further study brain development. 
Table 4.1. The number of pig genes expressed (mean FPKM $<10$ ) in the fetal brain but not expressed (mean FPKM <1) in the placenta or expressed in the placenta but not the fetal brain of pigs at gestation day (d) 60 and 90.

\begin{tabular}{|l|l|l|}
\hline Gestation Day & $\begin{array}{l}\text { Expressed in placenta } \\
\text { not in brain }\end{array}$ & $\begin{array}{l}\text { Expressed in brain } \\
\text { not in placenta }\end{array}$ \\
\hline $\mathrm{d} 60$ & 87 & 553 \\
\hline $\mathrm{d} 90$ & 80 & 554 \\
\hline
\end{tabular}


Table 4.2. The number of pig genes at gestation day (d) 60 and 90 expressed (mean FPKM > 10) in the mouse fetal brain but not expressed (mean FPKM $<1$ ) in the placenta or expressed in the mouse placenta but not the fetal brain.

\begin{tabular}{|l|l|l|}
\hline Gestation Day & $\begin{array}{l}\text { Pig genes expressed in } \\
\text { the mouse placenta but } \\
\text { not in the fetal brain }\end{array}$ & $\begin{array}{l}\text { Pig genes expressed in } \\
\text { the mouse fetal brain } \\
\text { but not in the placenta }\end{array}$ \\
\hline $\mathrm{d} 60$ & 92 & 171 \\
\hline $\mathrm{d} 90$ & 80 & 553 \\
\hline
\end{tabular}


Table 4.3. The number and type of homology genes between pigs and mice.

\begin{tabular}{|l|l|}
\hline Gene homology type & Number of Genes \\
\hline One to one & 15376 \\
\hline One to many & 2541 \\
\hline Many to many & 5193 \\
\hline
\end{tabular}


Figure 4.1. Venn diagrams comparing the pig genes expressed (mean FPKM<10) in the fetal brain but not expressed (mean FPKM $<1$ ) in the placenta (A) at gestation day (d) 60 and d90 or expressed in the placenta but not expressed in the fetal brain at d60 and d90 (B).

A

d60 Brain

d90 Brain

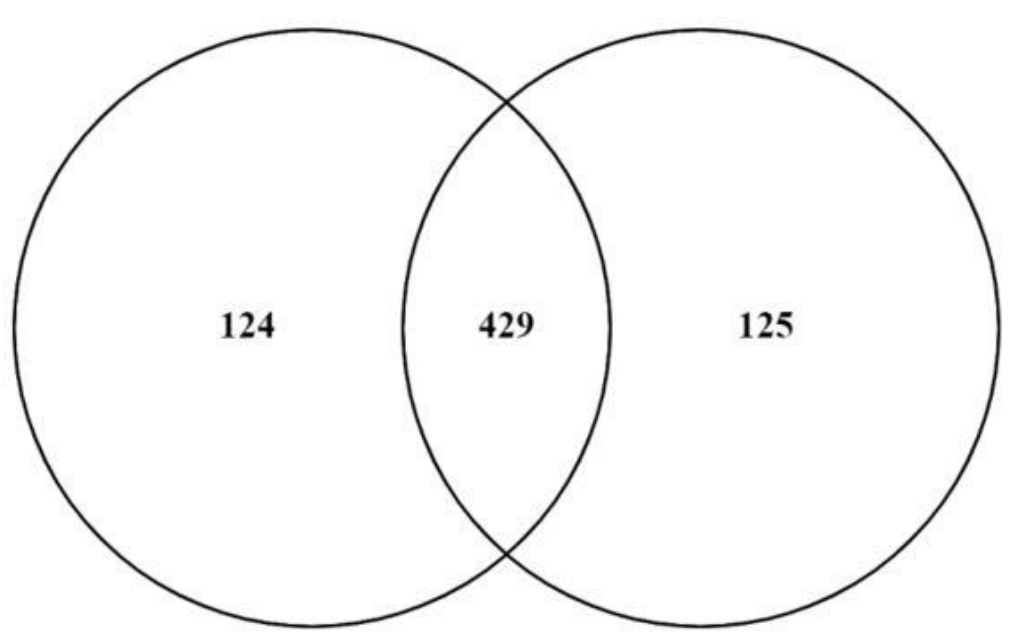

B d60 Placenta

d90 Placenta

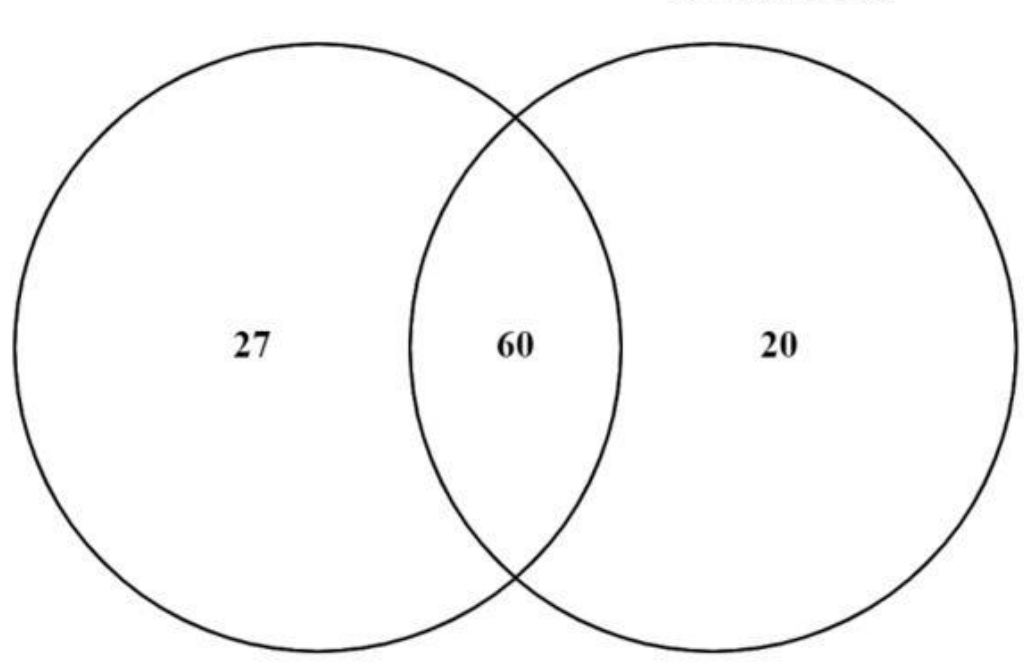


Figure 4.2. Venn diagrams comparing the number of genes expressed (mean FPKM $<10$ ) in the pig and mouse fetal brain but not expressed (mean FPKM <1) in the placenta $(\mathbf{A})$ or expressed in the placenta but not expressed in the fetal brain $(\mathbf{B})$.

A

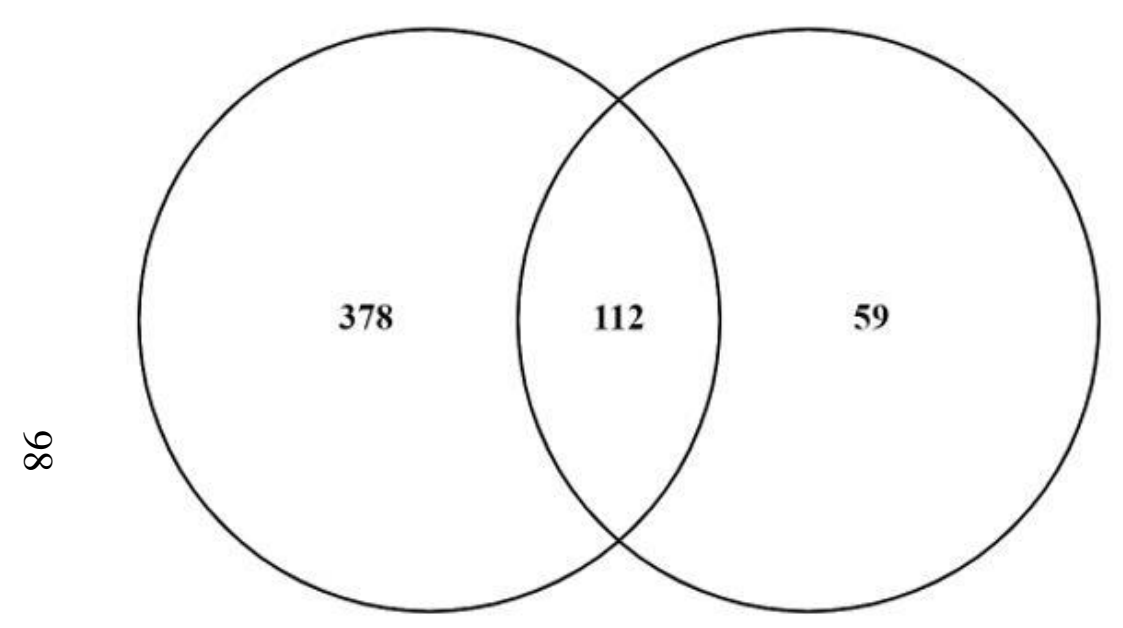

B

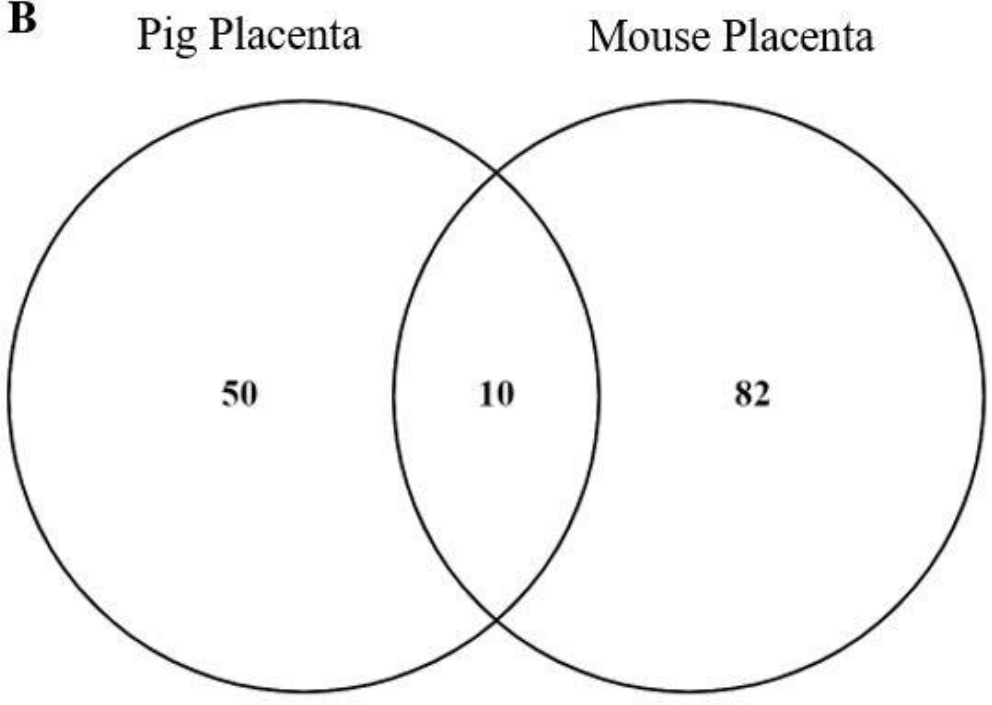


Figure 4.3. Venn diagrams comparing the 1:1 orthologous genes and the number of genes expressed (mean FPKM $<10)$ in the pig fetal brain but not expressed (mean FPKM <1) in the placenta at gestation day (d) 60 and d90 (A) and 1:1 orthologous genes compared to genes expressed in the pig placenta but not expressed in the fetal brain at d60 and d90 (B).

\section{A d60 Pig Brain}

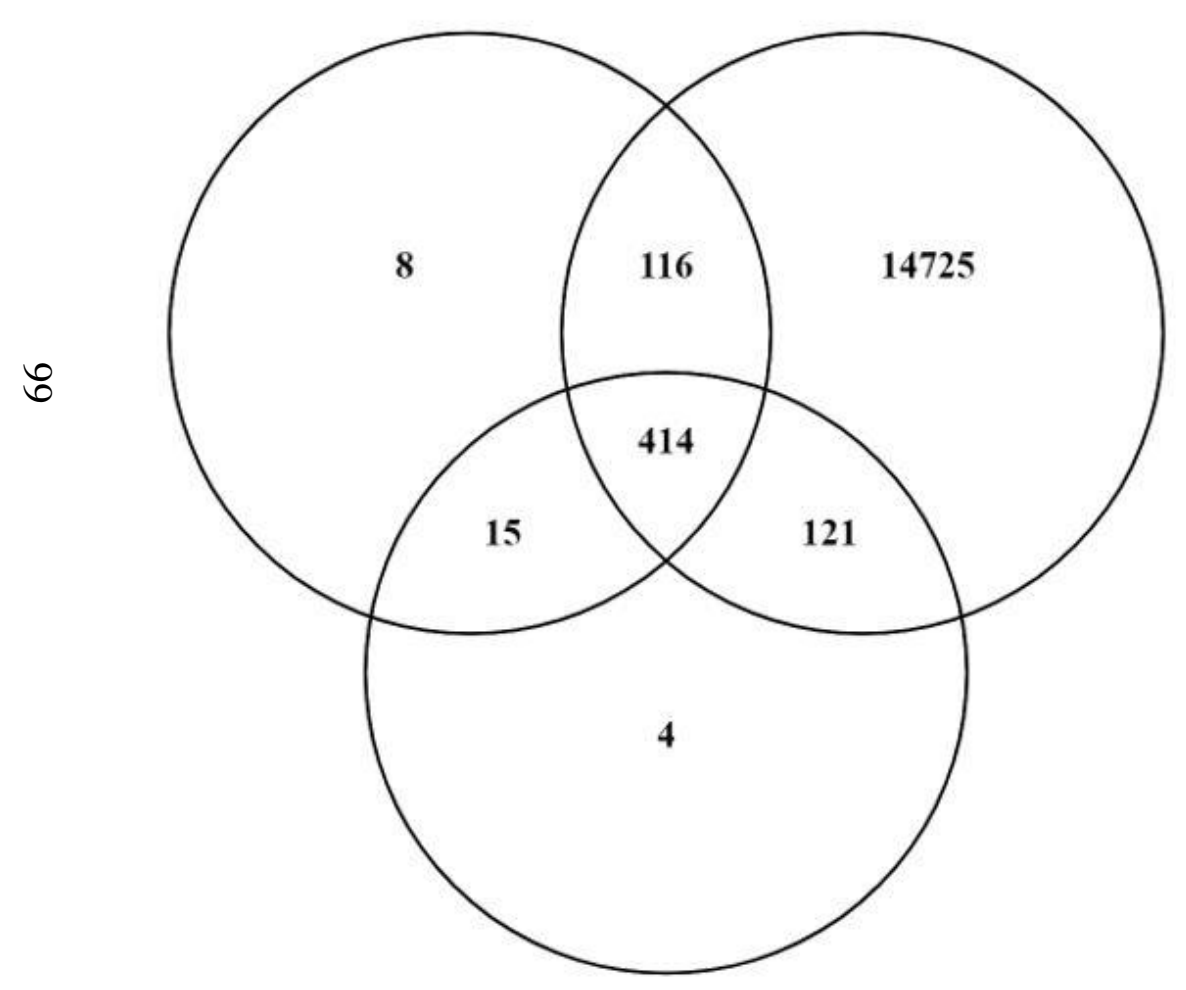

d90 Pig Brain

\section{B d60 Pig Placenta \\ 1:1 Genes}

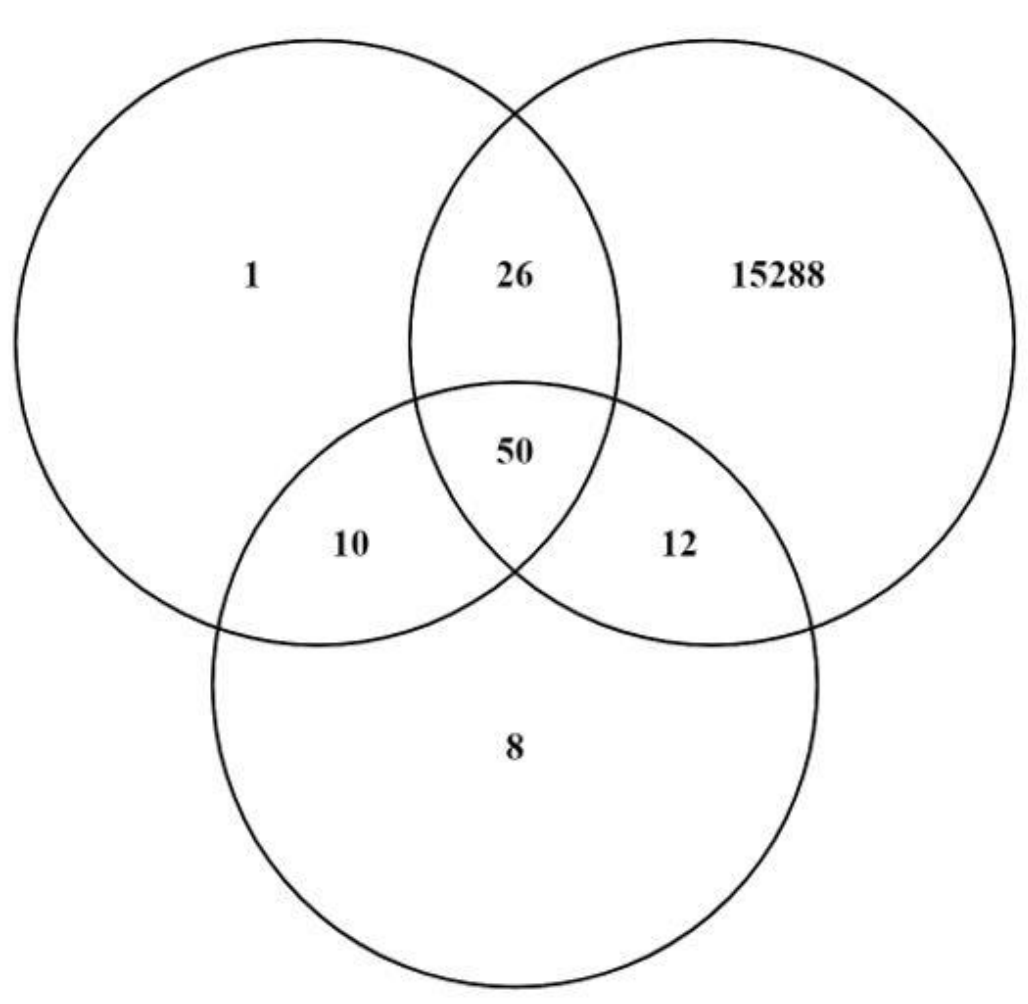

d90 Pig Placenta 
Figure 4.4. Description of one-to-one orthologous genes in pigs and mice, genes expressed in the fetal brain or placenta of both species and the common genes and transcription factor.

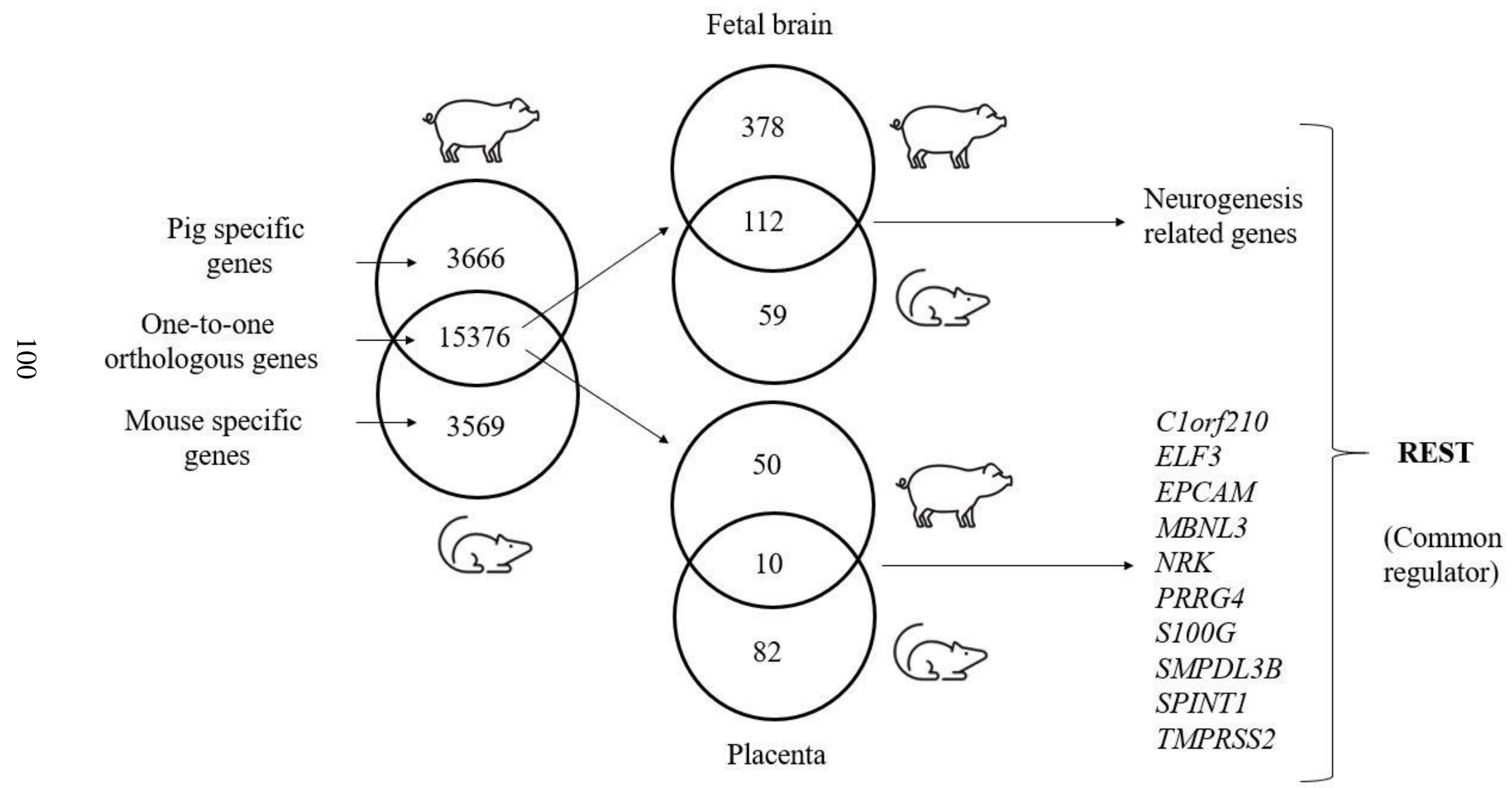




\section{Literature Cited}

Ackerman, S. 1992. The development and shaping of the brain. National Academies Press. Available from: https://www.ncbi.nlm.nih.gov/books/NBK234146/.

Ackermann, M., L. Chao, C. T. Bergstrom, and M. Doebeli. 2007. On the evolutionary origin of aging. Aging Cell. 6:235-244. doi:10.1111/j.1474-9726.2007.00281.x.

Aguilaniu, H. 2015. The mysterious relationship between reproduction and longevity. Worm. 4. doi:10.1080/21624054.2015.1020276. Available from: https://www.ncbi.nlm.nih.gov/pmc/articles/PMC4588167/

Alur, P. 2019. Sex differences in nutrition, growth, and metabolism in preterm infants. Front Pediatr. 7:22. doi:10.3389/fped.2019.00022.

Anastasiadi, D., A. Esteve-Codina, and F. Piferrer. 2018. Consistent inverse correlation between DNA methylation of the first intron and gene expression across tissues and species. Epigenetics \& Chromatin. 11:37. doi:10.1186/s13072-018-0205-1.

Antonson, A. M., B. Balakrishnan, E. C. Radlowski, G. Petr, and R. W. Johnson. 2018. Altered hippocampal gene expression and morphology in fetal piglets following maternal respiratory viral infection. DNE. 40:104-119. doi:10.1159/000486850.

Ashburner, M., C. A. Ball, J. A. Blake, D. Botstein, H. Butler, J. M. Cherry, A. P. Davis, K. Dolinski, S. S. Dwight, J. T. Eppig, M. A. Harris, D. P. Hill, L. Issel-Tarver, A. Kasarskis, S. Lewis, J. C. Matese, J. E. Richardson, M. Ringwald, G. M. Rubin, and G. Sherlock. 2000. Gene ontology: tool for the unification of biology. The gene ontology consortium. Nat Genet. 25:25-29. doi:10.1038/75556. 
Avery, O. T., C. M. MacLeod, and M. McCarty. 1944. Studies on the chemical nature of the substance inducing transformation of pneumococcal types. J Exp Med. 79:137-158.

Bainbridge, M. N., R. L. Warren, M. Hirst, T. Romanuik, T. Zeng, A. Go, A. Delaney, M. Griffith, M. Hickenbotham, V. Magrini, E. R. Mardis, M. D. Sadar, A. S. Siddiqui, M. A. Marra, and S. J. Jones. 2006. Analysis of the prostate cancer cell line $\mathrm{LNCaP}$ transcriptome using a sequencing-by-synthesis approach. BMC Genomics. 7:246. doi:10.1186/1471-2164-7-246.

Bakker, J., and M. J. Baum. 2008. Role for estradiol in female-typical brain and behavioral sexual differentiation. Front Neuroendocrinol. 29:1-16. doi:10.1016/j.yfrne.2007.06.001.

Barker, D. J. P. 2007. The origins of the developmental origins theory. J Intern Med. 261:412-417. doi:10.1111/j.1365-2796.2007.01809.x.

Barker, D. J. P., and C. Osmond. 1986. Infant mortality, childhood nutrition, and ischaemic heart disease in England and Wales. The Lancet. 327:1077-1081. doi:10.1016/S0140-6736(86)91340-1.

Barker, D. J. P., C. Osmond, K. L. Thornburg, E. Kajantie, and J. G. Eriksson. 2011. The lifespan of men and the shape of their placental surface at birth. Placenta. 32:783787. doi:10.1016/j.placenta.2011.07.031.

Barrett, K. E., S. Boitano, and S. M. Barman. 2010. Ganong's Review of Medical Physiology (23rd Edition). McGraw-Hill Professional Publishing, New York, 
USA. Available from:

http://public.ebookcentral.proquest.com/choice/publicfullrecord.aspx?p=4657620

Baxter, E. M., S. Jarvis, J. Palarea-Albaladejo, and S. A. Edwards. 2012. The weaker sex? The propensity for male-biased piglet mortality. PLoS One. 7. doi:10.1371/journal.pone.0030318. Available from: https://www.ncbi.nlm.nih.gov/pmc/articles/PMC3260262/

Behura, S. K., A. M. Kelleher, and T. E. Spencer. 2019b. Evidence for functional interactions between the placenta and brain in pregnant mice. The FASEB Journal. 33:4261-4272. doi:https://doi.org/10.1096/fj.201802037R.

Behura, S. K., A. M. Kelleher, and T. E. Spencer. 2020. Regulation of uterine genes during the peri-implantation period, and its relationship to the maternal brain in gestating mice. Mol. Reprod. Dev. 87:482-492. doi:https://doi.org/10.1002/mrd.23338.

Behura, S. K., P. Dhakal, A. M. Kelleher, A. Balboula, A. Patterson, and T. E. Spencer. 2019a. The brain-placental axis: Therapeutic and pharmacological relevancy to pregnancy. Pharmacol Res. 149:104468. doi:10.1016/j.phrs.2019.104468.

Bernstein, A. I., and P. Jin. 2015. Throughput sequencing-based mapping of cytosine modifications. In: Y. G. Zheng, editor. Epigenetic Technological Applications. Academic Press, Boston. p. 39-53. Available from: http://www.sciencedirect.com/science/article/pii/B978012801080800003X 
Bianco-Miotto, T., J. M. Craig, Y. P. Gasser, S. J. van Dijk, and S. E. Ozanne. 2017. Epigenetics and DOHaD: from basics to birth and beyond. J Dev Orig Health Dis. 8:513-519. doi:10.1017/S2040174417000733.

Bocklandt, S., W. Lin, M. E. Sehl, F. J. Sánchez, J. S. Sinsheimer, S. Horvath, and E. Vilain. 2011. Epigenetic predictor of age. PLoS One. 6. doi:10.1371/journal.pone.0014821.

Bollen, P. J. A., A. K. Hansen, and A. K. O. Alstrup. 2010. The labratory swine. 2nd ed. CRC Press, Boca Raton, FL.

Bouchard, L. 2013. Epigenetics and fetal metabolic programming: a call for integrated research on larger cohorts. Diabetes. 62:1026-1028. doi:10.2337/db12-1763.

Brockschnieder, D. 2006. Ermin, A myelinating oligodendrocyte-specific protein that regulates cell morphology. J. Neurosci. 26:757-762. doi:10.1523/JNEUROSCI.4317-05.2006.

Bronson, S. L., and T. L. Bale. 2016. The placenta as a mediator of stress effects on neurodevelopmental reprogramming. NPP. 41:207-218. doi:10.1038/npp.2015.231.

Chen, J., Q. Zou, D. Lv, M. A. Raza, X. Wang, P. Li, Y. Chen, X. Xi, A. Wen, L. Zhu, G. Tang, M. Li, X. Li, and Y. Jiang. 2019. Comprehensive transcriptional profiling of porcine brain aging. Gene. 693:1-9. doi:10.1016/j.gene.2019.01.019.

Chen, W. G., Q. Chang, Y. Lin, A. Meissner, A. E. West, E. C. Griffith, R. Jaenisch, and M. E. Greenberg. 2003. Derepression of BDNF transcription involves calcium- 
dependent phosphorylation of MeCP2. Science. 302:885-889. doi:10.1126/science.1086446.

Chen, Y., B. Pal, J. E. Visvader, and G. K. Smyth. 2018. Differential methylation analysis of reduced representation bisulfite sequencing experiments using edgeR. F1000Res. 6:2055. doi:10.12688/f1000research.13196.2.

Cheng, Y., and G. M. Church. 2000. Biclustering of Expression Data. Proc Int Conf Intell Syst Mol Biol. 8:93-103.

Cheung, F., B. J. Haas, S. M. Goldberg, G. D. May, Y. Xiao, and C. D. Town. 2006. Sequencing Medicago truncatula expressed sequenced tags using 454 Life Sciences technology. BMC Genomics. 7:272. doi:10.1186/1471-2164-7-272.

Choi, M., J. Lee, M. T. Le, D. T. Nguyen, S. Park, N. Soundrarajan, K. M. Schachtschneider, J. Kim, J.-K. Park, J.-H. Kim, and C. Park. 2015. Genomewide analysis of DNA methylation in pigs using reduced representation bisulfite sequencing. DNA Res. 22:343-355. doi:10.1093/dnares/dsv017.

Choi, Y., H. Seo, J. Shim, M. Kim, and H. Ka. 2012. Regulation of S100G expression in the uterine endometrium during early pregnancy in pigs. Asian-Australas J Anim Sci. 25:44-51. doi:10.5713/ajas.2011.11305.

Christoforou, E. R., and A. N. Sferruzzi-Perri. 2020. Molecular mechanisms governing offspring metabolic programming in rodent models of in utero stress. Cell. Mol. Life Sci. 77:4861-4898. doi:10.1007/s00018-020-03566-z. 
Chung, W. C. J., and A. P. Auger. 2013. Gender differences in neurodevelopment and epigenetics. Pflugers Arch. 465:573-584. doi:10.1007/s00424-013-1258-4.

Clouard, C., M. C. Meunier-Salaün, and D. Val-Laillet. 2012. Food preferences and aversions in human health and nutrition: how can pigs help the biomedical research? Animal. 6:118-136. doi:10.1017/S1751731111001315.

Compere, S. J., and R. D. Palmiter. 1981. DNA methylation controls the inducibility of the mouse metallothionein-I gene lymphoid cells. Cell. 25:233-240. doi:10.1016/0092-8674(81)90248-8.

Conrad, M. S., R. N. Dilger, and R. W. Johnson. 2012. Brain growth of the domestic pig (Sus scrofa) from 2 to 24 weeks of age: a longitudinal MRI study. Dev Neurosci. 34:291-298. doi:10.1159/000339311.

Cooke, B. M., G. Tabibnia, and S. M. Breedlove. 1999. A brain sexual dimorphism controlled by adult circulating androgens. Proc Natl Acad Sci U S A. 96:75387540. doi:10.1073/pnas.96.13.7538.

Cortes, L. R., C. D. Cisternas, and N. G. Forger. 2019. Does gender leave an epigenetic imprint on the brain? Front. Neurosci. 13. doi:10.3389/fnins.2019.00173.

Cotton, A. M., E. M. Price, M. J. Jones, B. P. Balaton, M. S. Kobor, and C. J. Brown. 2015. Landscape of DNA methylation on the $\mathrm{X}$ chromosome reflects $\mathrm{CpG}$ density, functional chromatin state and X-chromosome inactivation. Hum Mol Genet. 24:1528-1539. doi:10.1093/hmg/ddu564. 
Cousins, R., and C. E. Wood. 2010. Expression of organic anion transporters 1 and 3 in the ovine fetal brain during the latter half of gestation. Neurosci Lett. 484:22-25. doi:10.1016/j.neulet.2010.08.008.

Cox, B., M. Kotlyar, A. I. Evangelou, V. Ignatchenko, A. Ignatchenko, K. Whiteley, I. Jurisica, S. L. Adamson, J. Rossant, and T. Kislinger. 2009. Comparative systems biology of human and mouse as a tool to guide the modeling of human placental pathology. Mol Syst Biol. 5:279. doi:10.1038/msb.2009.37.

Crawford, M. A., W. Doyle, and N. Meadows. 1987. Gender differences at birth and differences in fetal growth. Hum Reprod. 2:517-520. doi:10.1093/oxfordjournals.humrep.a136581.

Cumming, P., M. Møller, K. Benda, L. Minuzzi, S. Jakobsen, S. B. Jensen, B. Pakkenberg, A. K. Stark, J. B. Gramsbergen, M. F. Andreasen, and A. K. Olsen. 2007. A PET study of effects of chronic 3,4-methylenedioxymethamphetamine (MDMA, “ecstasy”) on serotonin markers in Göttingen minipig brain. Synapse. 61:478-487. doi:10.1002/syn.20377.

de la Torre-Ubieta, L., J. L. Stein, H. Won, C. K. Opland, D. Liang, D. Lu, and D. H. Geschwind. 2018. The dynamic landscape of open chromatin during human cortical neurogenesis. Cell. 172:289-304.e18. doi:10.1016/j.cell.2017.12.014.

de Magalhães, J. P., and G. M. Church. 2005. Genomes optimize reproduction: aging as a consequence of the developmental program. Physiology (Bethesda). 20:252-259. doi:10.1152/physiol.00010.2005. 
Denda, K., K. Nakao-Wakabayashi, N. Okamoto, N. Kitamura, J.-Y. Ryu, Y. Tagawa, T. Ichisaka, S. Yamanaka, and M. Komada. 2011. Nrk, an X-linked protein kinase in the germinal center kinase family, is required for placental development and fetoplacental induction of labor. J Biol Chem. 286:28802-28810. doi:10.1074/jbc.M111.258160.

Dhakal, P., A. M. Kelleher, S. K. Behura, and T. E. Spencer. 2020. Sexually dimorphic effects of forkhead box a2 (FOXA2) and uterine glands on decidualization and fetoplacental development. PNAS. 117:23952-23959. doi:10.1073/pnas.2014272117.

Dickerson, J., and J. Dobbing. 1967. Prenatal and postnatal growth and development of the central nervous system of the pig. Proc. R. Soc. Lond. B. 166:384-395. doi:10.1098/rspb.1967.0002.

Dilman, V. M. 1971. Age-associated elevation of hypothalamic, threshold to feedback control, and its role in development, ageine, and disease. Lancet. 1:1211-1219. doi:10.1016/s0140-6736(71)91721-1.

Dobbing, J. 1972. Vulnerable periods of brain development. In: Lipids, Malnutrition \& the Developing Brain. 3rd ed. Elsevier. p. 9-29.

Dobbing, J. 1990. Vulnerable periods in developing brain. In: Brain, behaviour, and iron in the infant diet. Springer. p. 1-17.

Dobbing, J., and J. Sands. 1979. Comparative aspects of the brain growth spurt. Early Hum. Dev. 3:79-83. doi:10.1016/0378-3782(79)90022-7. 
dos Reis, M., J. Inoue, M. Hasegawa, R. J. Asher, P. C. J. Donoghue, and Z. Yang. 2012. Phylogenomic datasets provide both precision and accuracy in estimating the timescale of placental mammal phylogeny. Proc Biol Sci. 279:3491-3500. doi:10.1098/rspb.2012.0683.

Duncan, C. G., S. A. Grimm, D. L. Morgan, P. R. Bushel, B. D. Bennett, J. D. Roberts, F. L. Tyson, B. A. Merrick, and P. A. Wade. 2018. Dosage compensation and DNA methylation landscape of the X chromosome in mouse liver. Sci Rep. 8:10138. doi:10.1038/s41598-018-28356-3.

Dyce, K., W. Sack, and C. J. G. Wensing. 2009. The nervous system. In: Textbook of Veterinary Anatomy. 4th ed. Saunders. p. 268-331.

Edgar, R. D., M. J. Jones, M. J. Meaney, G. Turecki, and M. S. Kobor. 2017. BECon: a tool for interpreting DNA methylation findings from blood in the context of brain. Transl Psychiatry. 7:e1187-e1187. doi:10.1038/tp.2017.171.

Ehrlich, M., M. A. Gama-Sosa, L. H. Huang, R. M. Midgett, K. C. Kuo, R. A. McCune, and C. Gehrke. 1982. Amount and distribution of 5-methylcytosine in human DNA from different types of tissues of cells. Nucleic Acids Res. 10:2709-2721. doi:10.1093/nar/10.8.2709

Emrich, S. J., W. B. Barbazuk, L. Li, and P. S. Schnable. 2007. Gene discovery and annotation using LCM-454 transcriptome sequencing. Genome Res. 17:69-73. doi:10.1101/gr.5145806. 
Engebretsen, S., and J. Bohlin. 2019. Statistical predictions with glmnet. Clin Epigenet. 11:123. doi:10.1186/s13148-019-0730-1.

Erraji-Benchekroun, L., M. D. Underwood, V. Arango, H. Galfalvy, P. Pavlidis, P. Smyrniotopoulos, J. J. Mann, and E. Sibille. 2005. Molecular aging in human prefrontal cortex is selective and continuous throughout adult life. Biol. Psychiatry. 57:549-558. doi:10.1016/j.biopsych.2004.10.034.

Eventov-Friedman, S., H. Katchman, E. Shezen, A. Aronovich, D. Tchorsh, B. Dekel, E. Freud, and Y. Reisner. 2005. Embryonic pig liver, pancreas, and lung as a source for transplantation: Optimal organogenesis without teratoma depends on distinct time windows. PNAS. 102:2928-2933. doi:10.1073/pnas.0500177102.

Fang, F., S. Turcan, A. Rimner, A. Kaufman, D. Giri, L. G. T. Morris, R. Shen, V. Seshan, Q. Mo, A. Heguy, S. B. Baylin, N. Ahuja, A. Viale, J. Massague, L. Norton, L. T. Vahdat, M. E. Moynahan, and T. A. Chan. 2011. Breast cancer methylomes establish an epigenomic foundation for metastasis. Sci Transl Med. 3:75ra25. doi:10.1126/scitranslmed.3001875.

Fang, H., C. M. Disteche, and J. B. Berletch. 2019. X Inactivation and escape: epigenetic and structural features. Front. Cell Dev. Biol. 7. doi:10.3389/fcell.2019.00219. Available from: https://www.frontiersin.org/articles/10.3389/fcell.2019.00219/full

Farré, P., M. J. Jones, M. J. Meaney, E. Emberly, G. Turecki, and M. S. Kobor. 2015. Concordant and discordant DNA methylation signatures of aging in human blood and brain. Epigenetics Chromatin. 8:19. doi:10.1186/s13072-015-0011-y. 
Feltes, B. C., J. de F. Poloni, and D. Bonatto. 2015. Development and aging: two opposite but complementary phenomena. Interdiscip Top Gerontol. 40:74-84. doi:10.1159/000364932.

Feng, J., Y. Zhou, S. L. Campbell, T. Le, E. Li, J. D. Sweatt, A. J. Silva, and G. Fan. 2010. Dnmt1 and Dnmt3a maintain DNA methylation and regulate synaptic function in adult forebrain neurons. Nat. Neurosci. 13:423-430. doi:10.1038/nn.2514.

Foxcroft, G. R., W. T. Dixon, S. Novak, C. T. Putman, S. C. Town, and M. D. A. Vinsky. 2006. The biological basis for prenatal programming of postnatal performance in pigs. J Anim Sci. 84 Suppl:E105-112. doi:10.2527/2006.8413_supple105x.

Franzén, O., L.-M. Gan, and J. L. M. Björkegren. 2019. PanglaoDB: a web server for exploration of mouse and human single-cell RNA sequencing data. Database (Oxford). 2019:baz046. doi:10.1093/database/baz046.

Freytag, V., T. Carrillo-Roa, A. Milnik, P. G. Sämann, V. Vukojevic, D. Coynel, P. Demougin, T. Egli, L. Gschwind, F. Jessen, E. Loos, W. Maier, S. G. RiedelHeller, M. Scherer, C. Vogler, M. Wagner, E. B. Binder, D. J.-F. de Quervain, and A. Papassotiropoulos. 2017. A peripheral epigenetic signature of immune system genes is linked to neocortical thickness and memory. Nat Commun. 8:15193. doi:10.1038/ncomms15193.

Gage, S. H., M. R. Munafò, and G. Davey Smith. 2016. Causal inference in developmental origins of health and disease (DOHaD) research. Annu Rev Psychol. 67:567-585. doi:10.1146/annurev-psych-122414-033352. 
Galili, T. 2015. dendextend: an R package for visualizing, adjusting and comparing trees of hierarchical clustering. Bioinformatics. 31:3718-3720. doi:10.1093/bioinformatics/btv428.

Galjaard, S., L. Ameye, C. C. Lees, A. Pexsters, T. Bourne, D. Timmerman, and R. Devlieger. 2019. Sex differences in fetal growth and immediate birth outcomes in a low-risk Caucasian population. Biol Sex Differ. 10:48-59. doi:10.1186/s13293019-0261-7.

Giuffra, E., J. M. Kijas, V. Amarger, O. Carlborg, J. T. Jeon, and L. Andersson. 2000. The origin of the domestic pig: independent domestication and subsequent introgression. Genetics. 154:1785-1791.

Gluckman, P. D., M. A. Hanson, C. Cooper, and K. L. Thornburg. 2008. Effect of in utero and early-life conditions on adult health and disease. N Engl J Med. 359:6173. doi:10.1056/NEJMra0708473.

Gopalakrishnan, V. 2009. REST and the RESTless: in stem cells and beyond. Future Neurol. 4:317-329. doi:10.2217/fnl.09.1.

Gorkin, D. U., I. Barozzi, Y. Zhao, Y. Zhang, H. Huang, A. Y. Lee, B. Li, J. Chiou, A. Wildberg, B. Ding, B. Zhang, M. Wang, J. S. Strattan, J. M. Davidson, Y. Qiu, V. Afzal, J. A. Akiyama, I. Plajzer-Frick, C. S. Novak, M. Kato, T. H. Garvin, Q. T. Pham, A. N. Harrington, B. J. Mannion, E. A. Lee, Y. Fukuda-Yuzawa, Y. He, S. Preissl, S. Chee, J. Y. Han, B. A. Williams, D. Trout, H. Amrhein, H. Yang, J. M. Cherry, W. Wang, K. Gaulton, J. R. Ecker, Y. Shen, D. E. Dickel, A. Visel, L. A. 
Pennacchio, and B. Ren. 2020. An atlas of dynamic chromatin landscapes in mouse fetal development. Nature. 583:744-751. doi:10.1038/s41586-020-2093-3.

Groenen, M. A. M., A. L. Archibald, H. Uenishi, C. K. Tuggle, Y. Takeuchi, M. F. Rothschild, C. Rogel-Gaillard, C. Park, D. Milan, H.-J. Megens, S. Li, D. M. Larkin, H. Kim, L. A. F. Frantz, M. Caccamo, H. Ahn, B. L. Aken, A. Anselmo, C. Anthon, L. Auvil, B. Badaoui, C. W. Beattie, C. Bendixen, D. Berman, F. Blecha, J. Blomberg, L. Bolund, M. Bosse, S. Botti, Z. Bujie, M. Bystrom, B. Capitanu, D. Carvalho-Silva, P. Chardon, C. Chen, R. Cheng, S.-H. Choi, W. Chow, R. C. Clark, C. Clee, R. P. M. A. Crooijmans, H. D. Dawson, P. Dehais, F. De Sapio, B. Dibbits, N. Drou, Z.-Q. Du, K. Eversole, J. Fadista, S. Fairley, T. Faraut, G. J. Faulkner, K. E. Fowler, M. Fredholm, E. Fritz, J. G. R. Gilbert, E. Giuffra, J. Gorodkin, D. K. Griffin, J. L. Harrow, A. Hayward, K. Howe, Z.-L. Hu, S. J. Humphray, T. Hunt, H. Hornshøj, J.-T. Jeon, P. Jern, M. Jones, J. Jurka, H. Kanamori, R. Kapetanovic, J. Kim, J.-H. Kim, K.-W. Kim, T.-H. Kim, G. Larson, K. Lee, K.-T. Lee, R. Leggett, H. A. Lewin, Y. Li, W. Liu, J. E. Loveland, Y. Lu, J. K. Lunney, J. Ma, O. Madsen, K. Mann, L. Matthews, S. McLaren, T. Morozumi, M. P. Murtaugh, J. Narayan, D. T. Nguyen, P. Ni, S.-J. Oh, S. Onteru, et al. 2012. Analyses of pig genomes provide insight into porcine demography and evolution. Nature. 491:393-398. doi:10.1038/nature11622.

Gualtieri, T., and R. E. Hicks. 1985. An immunoreactive theory of selective male affliction. Behav Brain Sci. 8:427-441. doi:10.1017/S0140525X00001023. 
Gupta, S. K., P. Gressens, and S. Mani. 2009. NRSF downregulation induces neuronal differentiation in mouse embryonic stem cells. Differentiation. 77:19-28. doi:10.1016/j.diff.2008.09.001.

Hammerman, M. R. 2014. Classic and current opinion in embryonic organ transplantation. Curr Opin Organ Transplant. 19:133-139. doi:10.1097/MOT.0000000000000054.

Hannum, G., J. Guinney, L. Zhao, L. Zhang, G. Hughes, S. Sadda, B. Klotzle, M. Bibikova, J.-B. Fan, Y. Gao, R. Deconde, M. Chen, I. Rajapakse, S. Friend, T. Ideker, and K. Zhang. 2013. Genome-wide methylation profiles reveal quantitative views of human aging rates. Mol Cell. 49:359-367. doi:10.1016/j.molcel.2012.10.016.

Hard, D. L., and L. L. Anderson. 1979. Maternal starvation and progesterone secretion, litter size, and growth in the pig. Am J Physiol. 237:E273-280. doi:10.1152/ajpendo.1979.237.3.E273.

Héberlé, É., and A. F. Bardet. 2019. Sensitivity of transcription factors to DNA methylation. Essays Biochem. 63:727-741. doi:10.1042/EBC20190033.

Heindel, J. J., and L. N. Vandenberg. 2015. Developmental origins of health and disease: a paradigm for understanding disease cause and prevention. Curr Opin Pediatr. 27:248-253. doi:10.1097/MOP.0000000000000191.

Heinz, S., C. Benner, N. Spann, E. Bertolino, Y. C. Lin, P. Laslo, J. X. Cheng, C. Murre, H. Singh, and C. K. Glass. 2010. Simple combinations of lineage-determining 
transcription factors prime cis-regulatory elements required for macrophage and B cell identities. Mol Cell. 38:576-589. doi:10.1016/j.molcel.2010.05.004.

Hoffe, B., and M. R. Holahan. 2019. The use of pigs as a translational model for studying neurodegenerative diseases. Front Physiol. 10:838-845. doi:10.3389/fphys.2019.00838.

Holliday, R., and J. E. Pugh. 1975. DNA modification mechanisms and gene activity during development. Science. 187:226-232. doi:10.1126/science.187.4173.226.

Horvath, S. 2013. DNA methylation age of human tissues and cell types. Genome Biol. 14:3156. doi:10.1186/gb-2013-14-10-r115.

Horvath, S., M. Gurven, M. E. Levine, B. C. Trumble, H. Kaplan, H. Allayee, B. R. Ritz, B. Chen, A. T. Lu, T. M. Rickabaugh, B. D. Jamieson, D. Sun, S. Li, W. Chen, L. Quintana-Murci, M. Fagny, M. S. Kobor, P. S. Tsao, A. P. Reiner, K. L. Edlefsen, D. Absher, and T. L. Assimes. 2016. An epigenetic clock analysis of race/ethnicity, sex, and coronary heart disease. Genome Biol. 17:171-193. doi:10.1186/s13059-016-1030-0.

Hoshino, A., S. Horvath, A. Sridhar, A. Chitsazan, and T. A. Reh. 2019. Synchrony and asynchrony between an epigenetic clock and developmental timing. Sci Rep. 9:3770-3781. doi:10.1038/s41598-019-39919-3.

Hosoya, K., and M. Tachikawa. 2011. Roles of organic anion/cation transporters at the blood-brain and blood-cerebrospinal fluid barriers involving uremic toxins. Clin Exp Nephrol. 15:478-485. doi:10.1007/s10157-011-0460-y. 
Hotchkiss, R. D. 1948. The quantitative separation of purines, pyrimidines, and nucleosides by paper chromatography. J Biol Chem. 175:315-332.

Jagust, W. J. 2016. Early life sets the stage for aging. Proc Natl Acad Sci USA. 113:9148-9150. doi:10.1073/pnas.1609720113.

Jang, Y. D., Y. L. Ma, and M. D. Lindemann. 2014. Intrauterine position affects fetal weight and crown-rump length throughout gestation. J Anim Sci. 92:4400-4406. doi:10.2527/jas.2014-7762.

Janky, R., A. Verfaillie, H. Imrichová, B. V. de Sande, L. Standaert, V. Christiaens, G. Hulselmans, K. Herten, M. N. Sanchez, D. Potier, D. Svetlichnyy, Z. K. Atak, M. Fiers, J.-C. Marine, and S. Aerts. 2014. iRegulon: from a gene list to a gene regulatory network using large motif and track collections. PLoS Comput. Biol. 10:e1003731. doi:10.1371/journal.pcbi.1003731.

Jansson, T., L. Myatt, and T. L. Powell. 2009. The role of trophoblast nutrient and ion transporters in the development of pregnancy complications and adult disease. Curr Vasc Pharmacol. 7:521-533. doi:10.2174/157016109789043982.

Jaquemar, D., S. Kupriyanov, M. Wankell, J. Avis, K. Benirschke, H. Baribault, and R. G. Oshima. 2003. Keratin 8 protection of placental barrier function. J Cell Biol. 161:749-756. doi:10.1083/jcb.200210004.

Jarmasz, J. S., H. Stirton, J. R. Davie, and M. R. Del Bigio. 2019. DNA methylation and histone post-translational modification stability in post-mortem brain tissue. Clin Epigenetics. 11:5-27. doi:10.1186/s13148-018-0596-7. 
Jennings, D. 2004. Supraspinal control of posture and movement. In: W. Reece, editor. Dukes' Physiology of Domestic Animals. 12th ed. Comstock Publishing Associates. p. 904-920.

Jensen Peña, C., C. Monk, and F. A. Champagne. 2012. Epigenetic effects of prenatal stress on $11 \beta$-hydroxysteroid dehydrogenase- 2 in the placenta and fetal brain. PLoS One. 7:e39791. doi:10.1371/journal.pone.0039791.

Ji, Y., Z. Wu, Z. Dai, X. Wang, J. Li, B. Wang, and G. Wu. 2017. Fetal and neonatal programming of postnatal growth and feed efficiency in swine. J. Anim. Sci. Biotechnol. 8:42. doi:10.1186/s40104-017-0173-5.

Jia, T., C. Chu, Y. Liu, J. van Dongen, E. Papastergios, N. J. Armstrong, M. E. Bastin, T. Carrillo-Roa, A. den Braber, M. Harris, R. Jansen, J. Liu, M. Luciano, A. P. S. Ori, R. Roiz Santiañez, B. Ruggeri, D. Sarkisyan, J. Shin, K. Sungeun, D. Tordesillas Gutiérrez, D. van’t Ent, D. Ames, E. Artiges, G. Bakalkin, T. Banaschewski, A. L. W. Bokde, H. Brodaty, U. Bromberg, R. Brouwer, C. Büchel, E. Burke Quinlan, W. Cahn, G. I. de Zubicaray, S. Ehrlich, T. J. Ekström, H. Flor, J. H. Fröhner, V. Frouin, H. Garavan, P. Gowland, A. Heinz, J. Hoare, B. Ittermann, N. Jahanshad, J. Jiang, J. B. Kwok, N. G. Martin, J.-L. Martinot, K. A. Mather, K. L. McMahon, A. F. McRae, F. Nees, D. Papadopoulos Orfanos, T. Paus, L. Poustka, P. G. Sämann, P. R. Schofield, M. N. Smolka, D. J. Stein, L. T. Strike, J. Teeuw, A. Thalamuthu, J. Trollor, H. Walter, J. M. Wardlaw, W. Wen, R. Whelan, L. G. Apostolova, E. B. Binder, D. I. Boomsma, V. Calhoun, B. Crespo-Facorro, I. J. Deary, H. Hulshoff Pol, R. A. Ophoff, Z. Pausova, P. S. 
Sachdev, A. Saykin, M. J. Wright, P. M. Thompson, G. Schumann, and S.

Desrivières. 2019. Epigenome-wide meta-analysis of blood DNA methylation and its association with subcortical volumes: findings from the ENIGMA epigenetics working group. Mol Psychiatry. 1-12. doi:10.1038/s41380-019-0605-z.

Johnson, A. A., K. Akman, S. R. G. Calimport, D. Wuttke, A. Stolzing, and J. P. de Magalhães. 2012. The role of DNA methylation in aging, rejuvenation, and agerelated disease. Rejuvenation Res. 15:483-494. doi:10.1089/rej.2012.1324.

Justice, E. D., S. J. Barnum, and T. Kidd. 2017. The WAGR syndrome gene PRRG4 is a functional homologue of the commissureless axon guidance gene. PLoS Genet. 13. doi:10.1371/journal.pgen.1006865. Available from: https://www.ncbi.nlm.nih.gov/pmc/articles/PMC5578492/

Jylhävä, J., N. L. Pedersen, and S. Hägg. 2017. Biological age predictors. EBioMedicine. 21:29-36. doi:10.1016/j.ebiom.2017.03.046.

Kalisch-Smith, J. I., D. G. Simmons, M. Pantaleon, and K. M. Moritz. 2017. Sex differences in rat placental development: from pre-implantation to late gestation. Biol. Sex Differ. 8:17-29. doi:10.1186/s13293-017-0138-6.

Kanekar, S., and C. Gustas. 2011. Metabolic disorders of the brain: part I. Semin Ultrasound CT MR. 32:590-614. doi:10.1053/j.sult.2011.08.003.

Karolchik, D., A. S. Hinrichs, T. S. Furey, K. M. Roskin, C. W. Sugnet, D. Haussler, and W. J. Kent. 2004. The UCSC table browser data retrieval tool. Nucleic Acids Res. 32:D493-496. doi:10.1093/nar/gkh103. 
Kim, T. S., C. Heinlein, R. C. Hackman, and P. S. Nelson. 2006. Phenotypic analysis of mice lacking the Tmprss2-Encoded Protease. Mol Cell Biol. 26:965-975. doi:10.1128/MCB.26.3.965-975.2006.

Kinder, HollyA., EmilyW. Baker, and FranklinD. West. 2019. The pig as a preclinical traumatic brain injury model: current models, functional outcome measures, and translational detection strategies. Neural Regen Res. 14:413-424. doi:10.4103/1673-5374.245334.

Knox, R. V. 2014. Impact of swine reproductive technologies on pig and global food production. Adv Exp Med Biol. 752:131-160. doi:10.1007/978-1-4614-8887-3_7.

Kraemer, S. 2000. The fragile male. BMJ. 321:1609-1612. doi:https://doi.org/10.1136/bmj.321.7276.1609.

Krueger, F., and S. R. Andrews. 2011. Bismark: a flexible aligner and methylation caller for Bisulfite-Seq applications. Bioinformatics. 27:1571-1572. doi:10.1093/bioinformatics/btr167.

Kumamoto, T., and S. Oshio. 2013. Effect of fetal exposure to bisphenol A on brain mediated by X-chromosome inactivation. J Toxicol Sci. 38:485-494. doi: $10.2131 /$ jts.38.485.

Kumamoto, T., N. Tsukue, H. Takano, K. Takeda, and S. Oshio. 2013. Fetal exposure to diesel exhaust affects $\mathrm{X}$-chromosome inactivation factor expression in mice. $\mathrm{J}$ Toxicol Sci. 38:245-254. doi:10.2131/jts.38.245. 
Laber, K. E., M. T. Whary, S. A. Bingel, J. A. Goodrich, A. C. Smith, and M. M. Swindle. 2015. Biology and diseases of swine. In: Laboratory Animal Medicine. 3rd ed. Elsevier, Amsterdam, Netherlands.

Lampl, M., F. Gotsch, J. P. Kusanovic, R. Gomez, J. K. Nien, E. A. Frongillo, and R. Romero. 2010. Sex differences in fetal growth responses to maternal height and weight. Am J Hum Biol. 22:431-443. doi:10.1002/ajhb.21014.

Larsen, M., C. R. Bjarkam, K. Østergaard, M. J. West, and J. C. Sørensen. 2004. The anatomy of the porcine subthalamic nucleus evaluated with immunohistochemistry and design-based stereology. Anat Embryol. 208:239247. doi:10.1007/s00429-004-0395-0.

Laufer, B. I., K. E. Neier, A. E. Valenzuela, D. H. Yasui, R. J. Schmidt, P. J. Lein, and J. M. LaSalle. 2021. Genome-Wide DNA methylation profiles of neurodevelopmental disorder genes in mouse placenta and fetal brain following prenatal exposure to polychlorinated biphenyls. bioRxiv. 2021.05.27.446011. doi:10.1101/2021.05.27.446011.

Lee, K.-S., Y. Cao, H. E. Witwicka, S. Tom, S. J. Tapscott, and E. H. Wang. 2010. RNAbinding protein muscleblind-like 3 (MBNL3) disrupts myocyte enhancer factor 2 (Mef2) $\beta$-exon splicing. J Biol Chem. 285:33779-33787. doi:10.1074/jbc.M110.124255.

Lee, Y., S. Choufani, R. Weksberg, S. L. Wilson, V. Yuan, A. Burt, C. Marsit, A. T. Lu, B. Ritz, J. Bohlin, H. K. Gjessing, J. R. Harris, P. Magnus, A. M. Binder, W. P. Robinson, A. Jugessur, and S. Horvath. 2019. Placental epigenetic clocks: 
estimating gestational age using placental DNA methylation levels. Aging (Albany NY). 11:4238-4253. doi:10.18632/aging.102049.

Leenhouwers, J. I., E. F. Knol, P. N. de Groot, H. Vos, and T. van der Lende. 2002. Fetal development in the pig in relation to genetic merit for piglet survival. J Anim Sci. 80:1759-1770. doi:10.2527/2002.8071759x.

Leiser, R., and V. Dantzer. 1988. Structural and functional aspects of porcine placental microvasculature. Anat Embryol (Berl). 177:409-419. doi:10.1007/BF00304738.

Lester, B. M., and C. J. Marsit. 2018. Epigenetic mechanisms in the placenta related to infant neurodevelopment. Epigenomics. 10:321-333. doi:10.2217/epi-2016-0171.

Lewis, D. L., M. A. DeCamillis, C. R. Brunetti, G. Halder, V. A. Kassner, J. E. Selegue, S. Higgs, and S. B. Carroll. 1999. Ectopic gene expression and homeotic transformations in arthropods using recombinant Sindbis viruses. Curr. 9:12791287. doi:10.1016/S0960-9822(00)80049-4.

Li, M., L. Chen, J. Zhang, C. Xiong, and X. Li. 2020. The SARS-CoV-2 receptor ACE2 expression of maternal-fetal interface and fetal organs by single-cell transcriptome study. PLoS One. 15:4-15. doi:10.1371/journal.pone.0230295.

Liao, Y., G. K. Smyth, and W. Shi. 2014. featureCounts: an efficient general purpose program for assigning sequence reads to genomic features. Bioinformatics. 30:923-930. doi:10.1093/bioinformatics/btt656.

Lin, D., J. Chen, S. Ehrlich, J. R. Bustillo, N. Perrone-Bizzozero, E. Walton, V. P. Clark, Y.-P. Wang, J. Sui, Y. Du, B. C. Ho, C. S. Schulz, V. D. Calhoun, and J. Liu. 
2018. Cross-Tissue exploration of genetic and epigenetic effects on brain gray matter in schizophrenia. Schizophr Bull. 44:443-452. doi:10.1093/schbul/sbx068.

Lind, N. M., A. Moustgaard, J. Jelsing, G. Vajta, P. Cumming, and A. K. Hansen. 2007. The use of pigs in neuroscience: modeling brain disorders. Neurosci Biobehav Rev. 31:728-751. doi:10.1016/j.neubiorev.2007.02.003.

Lister, R., E. A. Mukamel, J. R. Nery, M. Urich, C. A. Puddifoot, N. D. Johnson, J. Lucero, Y. Huang, A. J. Dwork, M. D. Schultz, M. Yu, J. Tonti-Filippini, H. Heyn, S. Hu, J. C. Wu, A. Rao, M. Esteller, C. He, F. G. Haghighi, T. J. Sejnowski, M. M. Behrens, and J. R. Ecker. 2013. Global epigenomic reconfiguration during mammalian brain development. Science. 341:1237905. doi:10.1126/science.1237905.

Lister, R., M. Pelizzola, R. H. Dowen, R. D. Hawkins, G. Hon, J. Tonti-Filippini, J. R. Nery, L. Lee, Z. Ye, Q.-M. Ngo, L. Edsall, J. Antosiewicz-Bourget, R. Stewart, V. Ruotti, A. H. Millar, J. A. Thomson, B. Ren, and J. R. Ecker. 2009. Human DNA methylomes at base resolution show widespread epigenomic differences. Nature. 462:315-322. doi:10.1038/nature08514.

Lowsky, D. J., S. J. Olshansky, J. Bhattacharya, and D. P. Goldman. 2014. Heterogeneity in healthy aging. J Gerontol A Biol Sci Med Sci. 69:640-649. doi:10.1093/gerona/glt162.

Lu, T., Y. Pan, S.-Y. Kao, C. Li, I. Kohane, J. Chan, and B. Yankner. 2004. Gene regulation and DNA damage in the ageing human brain. Nature. 429:883-91. doi:10.1038/nature02661. 
Lye, P., E. Bloise, M. Javam, W. Gibb, S. J. Lye, and S. G. Matthews. 2015. Impact of bacterial and viral challenge on multidrug resistance in first- and third-trimester human placenta. The American Journal of Pathology. 185:1666-1675. doi:10.1016/j.ajpath.2015.02.013.

Martin, E., L. Smeester, P. A. Bommarito, M. R. Grace, K. Boggess, K. Kuban, M. R. Karagas, C. J. Marsit, T. M. O’Shea, and R. C. Fry. 2017. Sexual epigenetic dimorphism in the human placenta: implications for susceptibility during the prenatal period. Epigenomics. 9:267-278. doi:10.2217/epi-2016-0132.

Martin, G. M. 2005. Epigenetic drift in aging identical twins. PNAS. 102:10413-10414. doi:10.1073/pnas.0504743102.

Martinowich, K., D. Hattori, H. Wu, S. Fouse, F. He, Y. Hu, G. Fan, and Y. E. Sun. 2003. DNA methylation-related chromatin remodeling in activity-dependent BDNF gene regulation. Science. 302:890-893. doi:10.1126/science.1090842.

McCarthy, M. M. 2016. Sex differences in the developing brain as a source of inherent risk. Dialogues Clin Neurosci. 18:361-372. doi:10.31887/DCNS.2016.18.4/mmccarthy.

McCarthy, M. M., A. P. Arnold, G. F. Ball, J. D. Blaustein, and Geert. J. De Vries. 2012. Sex differences in the brain: the not so inconvenient truth. J Neurosci. 32:22412247. doi:10.1523/JNEUROSCI.5372-11.2012.

McCarthy, M. M., A. P. Auger, T. L. Bale, G. J. De Vries, G. A. Dunn, N. G. Forger, E. K. Murray, B. M. Nugent, J. M. Schwarz, and M. E. Wilson. 2009. The 
Epigenetics of sex differences in the brain. J. Neurosci. 29:12815-12823. doi:10.1523/JNEUROSCI.3331-09.2009.

McCarty, M., and O. T. Avery. 1946. Studies on the chemical nature of the substance inducing transformation of pneumococcal types: II. Effect of desoxyribonuclease on the biological activity of the transforming substance. J Exp Med. 83:89-96.

McPherson, R. L., F. Ji, G. Wu, J. R. Blanton Jr., and S. W. Kim. 2004. Growth and compositional changes of fetal tissues in pigs. J Anim Sci. 82:2534-2540. doi: $10.2527 / 2004.8292534 \mathrm{x}$.

Merid, S. K., A. Novoloaca, G. C. Sharp, L. K. Küpers, A. T. Kho, R. Roy, L. Gao, I. Annesi-Maesano, P. Jain, M. Plusquin, M. Kogevinas, C. Allard, F. O. Vehmeijer, N. Kazmi, L. A. Salas, F. I. Rezwan, H. Zhang, S. Sebert, D. Czamara, S. L. Rifas-Shiman, P. E. Melton, D. A. Lawlor, G. Pershagen, C. V. Breton, K. Huen, N. Baiz, L. Gagliardi, T. S. Nawrot, E. Corpeleijn, P. Perron, L. Duijts, E. A. Nohr, M. Bustamante, S. L. Ewart, W. Karmaus, S. Zhao, C. M. Page, Z. Herceg, M.-R. Jarvelin, J. Lahti, A. A. Baccarelli, D. Anderson, P. Kachroo, C. L. Relton, A. Bergström, B. Eskenazi, M. H. Soomro, P. Vineis, H. Snieder, L. Bouchard, V. W. Jaddoe, T. I. A. Sørensen, M. Vrijheid, S. H. Arshad, J. W. Holloway, S. E. Håberg, P. Magnus, T. Dwyer, E. B. Binder, D. L. DeMeo, J. M. Vonk, J. Newnham, K. G. Tantisira, I. Kull, J. L. Wiemels, B. Heude, J. Sunyer, W. Nystad, M. C. Munthe-Kaas, K. Räikkönen, E. Oken, R.-C. Huang, S. T. Weiss, J. M. Antó, J. Bousquet, A. Kumar, C. Söderhäll, C. Almqvist, A. Cardenas, O. Gruzieva, C.-J. Xu, S. E. Reese, J. Kere, P. Brodin, O. Solomon, M. Wielscher, N. 
Holland, A. Ghantous, M.-F. Hivert, J. F. Felix, G. H. Koppelman, S. J. London, and E. Melén. 2020. Epigenome-wide meta-analysis of blood DNA methylation in newborns and children identifies numerous loci related to gestational age.

Genome Med. 12:25. doi:10.1186/s13073-020-0716-9.

Meyer, P. E., F. Lafitte, and G. Bontempi. 2008. minet: A R/Bioconductor package for inferring large transcriptional networks using mutual information. BMC Bioinformatics. 9:461. doi:10.1186/1471-2105-9-461.

Mi, H., A. Muruganujan, J. T. Casagrande, and P. D. Thomas. 2013. Large-scale gene function analysis with the PANTHER classification system. Nat Protoc. 8:15511566. doi:10.1038/nprot.2013.092.

Michael, K., B. S. Ward, and W. M. Moore. 1983. Relationship of fetal to placental size: the pig model. Eur J Obstet Gynecol Reprod Biol. 16:53-62. doi:10.1016/00282243(83)90220-4.

Miller, C. A., S. L. Campbell, and J. D. Sweatt. 2008. DNA methylation and histone acetylation work in concert to regulate memory formation and synaptic plasticity. Neurobiol Learn Mem. 89:599-603. doi:10.1016/j.nlm.2007.07.016.

Mudd, A. T., and R. N. Dilger. 2017. Early-life nutrition and neurodevelopment: Use of the piglet as a translational model. Adv Nutr. 8:92-104. doi:10.3945/an.116.013243. 
Müller, H.-G., J.-M. Chiou, J. R. Carey, and J.-L. Want. 2002. Fertility and life span: Late children enhance female longevity. J. Gerontol. 57:B202-B206. doi:10.1093/gerona/57.5.B202.

Nagalakshmi, U., Z. Wang, K. Waern, C. Shou, D. Raha, M. Gerstein, and M. Snyder. 2008. The transcriptional landscape of the yeast genome defined by RNA Sequencing. Science. 320:1344-1349. doi:10.1126/science.1158441.

Nelissen, E. C. M., A. P. A. van Montfoort, J. C. M. Dumoulin, and J. L. H. Evers. 2011. Epigenetics and the placenta. Hum. Reprod. Update. 17:397-417. doi:10.1093/humupd/dmq052.

Nguyen, D. K., and C. M. Disteche. 2006. High expression of the mammalian X chromosome in brain. Brain Res. 1126:46-49. doi:10.1016/j.brainres.2006.08.053.

Niemann, H., and D. Rath. 2001. Progress in reproductive biotechnology in swine. Theriogenology. 56:1291-1304. doi:10.1016/s0093-691x(01)00630-6.

Nigam, S. K., K. T. Bush, G. Martovetsky, S.-Y. Ahn, H. C. Liu, E. Richard, V. Bhatnagar, and W. Wu. 2015. The Organic anion transporter (OAT) family: A systems biology perspective. Physiol Rev. 95:83-123. doi:10.1152/physrev.00025.2013.

Noden, D. M. and A. De Lahunta. 1985. Central nervous system and eye. In: The embryology of domestic animals. Williams \& Wilkins. p. 92-119. 
Numata, S., T. Ye, T. M. Hyde, X. Guitart-Navarro, R. Tao, M. Wininger, C. Colantuoni, D. R. Weinberger, J. E. Kleinman, and B. K. Lipska. 2012. DNA methylation signatures in development and aging of the human prefrontal cortex. Am J Hum Genet. 90:260-272. doi:10.1016/j.ajhg.2011.12.020.

O’Connor, T. G., J. Heron, J. Golding, and V. Glover. 2003. Maternal antenatal anxiety and behavioural/emotional problems in children: a test of a programming hypothesis. J Child Psychol Psychiatry. 44:1025-1036. doi:https://doi.org/10.1111/1469-7610.00187.

Oda, Y., Y. Muroishi, H. Misawa, and S. Suzuki. 2004. Comparative study of gene expression of cholinergic system-related molecules in the human spinal cord and term placenta. Neuroscience. 128:39-49. doi:10.1016/j.neuroscience.2004.06.002.

Oksbjerg, N., P. M. Nissen, M. Therkildsen, H. S. Møller, L. B. Larsen, M. Andersen, and J. F. Young. 2013. Meat science and muscle biology symposium: in utero nutrition related to fetal development, postnatal performance, and meat quality of pork. J Anim Sci. 91:1443-1453. doi:10.2527/jas.2012-5849.

Ooi, L., and I. C. Wood. 2007. Chromatin crosstalk in development and disease: lessons from REST. Nat. Rev. Genet. 8:544-554. doi:10.1038/nrg2100.

Pallayova, M., A. Brandeburova, and D. Tokarova. 2019. Update on sexual dimorphism in brain structure-function interrelationships: A literature review. Appl Psychophysiol Biofeedback. 44:271-284. doi:10.1007/s10484-019-09443-1. 
Parés-Casanova, P. M. 2013. Sexual size dimorphism in swine denies Rensch's rule. Asian J. Agric. Sci. 01:112-118.

Pavanello, S., M. Campisi, A. Fabozzo, G. Cibin, V. Tarzia, G. Toscano, and G. Gerosa. 2020. The biological age of the heart is consistently younger than chronological age. Sci. Rep. 10. doi:10.1038/s41598-020-67622-1.

Penkler, M., M. Hanson, R. Biesma, and R. Müller. 2019. DOHaD in science and society: emergent opportunities and novel responsibilities. J Dev Orig Health Dis. 10:268273. doi:10.1017/S2040174418000892.

Pinney, S. E., and R. A. Simmons. 2012. Metabolic programming, epigenetics, and gestational diabetes mellitus. Curr Diab Rep. 12:67-74. doi:10.1007/s11892-0110248-1.

Pique-Regi, R., R. Romero, A. L. Tarca, F. Luca, Y. Xu, A. Alazizi, Y. Leng, C.-D. Hsu, and N. Gomez-Lopez. 2020. Does the human placenta express the canonical cell entry mediators for SARS-CoV-2? bioRxiv. 2020.05.18.101485. doi:10.1101/2020.05.18.101485.

Pitale, S., and A. Sahasrabuddhe. 2011. Fetal origin of vascular aging. Indian J Endocrinol Metab. 15 Suppl 4:S289-297. doi:10.4103/2230-8210.86971.

Pronker, M. F., S. Lemstra, J. Snijder, A. J. R. Heck, D. M. E. Thies-Weesie, R. J. Pasterkamp, and B. J. C. Janssen. 2016. Structural basis of myelin-associated glycoprotein adhesion and signalling. Nat Commun. 7. doi:10.1038/ncomms13584. 
Qi, W., S. Gao, C. Liu, G. Lan, X. Yang, and Q. Guo. 2017. Diffusion tensor MR imaging characteristics of cerebral white matter development in fetal pigs. BMC Med Imaging. 17:50-59. doi:10.1186/s12880-017-0205-9.

Quinlan, A. R., and I. M. Hall. 2010. BEDTools: a flexible suite of utilities for comparing genomic features. Bioinformatics. 26:841-842. doi:10.1093/bioinformatics/btq033.

Rabaglino, M. B., M. Keller-Wood, and C. E. Wood. 2014. Transcriptomics of the late gestation ovine fetal brain: modeling the co-expression of immune marker genes. BMC Genomics. 15:1001-1009. doi:10.1186/1471-2164-15-1001.

Radovic, M., M. Ghalwash, N. Filipovic, and Z. Obradovic. 2017. Minimum redundancy maximum relevance feature selection approach for temporal gene expression data. BMC Bioinformatics. 18:9-22. doi:10.1186/s12859-016-1423-9.

Ratnu, V. S., M. R. Emami, and T. W. Bredy. 2017. Genetic and epigenetic factors underlying sex differences in the regulation of gene expression in the brain. $\mathrm{J}$ Neurosci Res. 95:301-310. doi:10.1002/jnr.23886.

Renegar, R. H., F. W. Bazer, and R. M. Roberts. 1982. Placental transport and distribution of uteroferrin in the fetal pig. Biol Reprod. 27:1247-1260. doi:10.1095/biolreprod27.5.1247.

Rohde Parfet, K. A., W. R. Lamberson, A. R. Rieke, T. C. Cantley, V. K. Ganjam, F. S. vom Saal, and B. N. Day. 1990. Intrauterine position effects in male and female 
swine: subsequent survivability, growth rate, morphology and semen characteristics. J Anim Sci. 68:179-185. doi:10.2527/1990.681179x.

Rosenfeld, C. 2021. The placenta-brain-axis. J. Neurosci. Res. 99:271-283. doi:10.1002/jnr.24603.

Rosenfeld, C. S. 2015. Sex-Specific placental responses in fetal development. Endocrinology. 156:3422-3434. doi:10.1210/en.2015-1227.

Rothkötter, H. J., E. Sowa, and R. Pabst. 2002. The pig as a model of developmental immunology. Hum Exp Toxicol. 21:533-536. doi:10.1191/0960327102ht293oa.

Ruchat, S. M., M. F. Hivert, and L. Bouchard. 2013. Epigenetic programming of obesity and diabetes by in utero exposure to gestational diabetes mellitus. Nutr Rev. 71 Suppl 1:S88-94. doi:10.1111/nure.12057.

Ryan, B. C., and J. G. Vandenbergh. 2002. Intrauterine position effects. Neurosci Biobehav Rev. 26:665-678. doi:10.1016/s0149-7634(02)00038-6.

Salomon, L. J., and C. Garel. 2007. Magnetic resonance imaging examination of the fetal brain. Ultrasound Obstet Gynecol. 30:1019-1032. doi:10.1002/uog.5176.

Sangkhae, V., and E. Nemeth. 2019. Placental iron transport: The mechanism and regulatory circuits. Free Radic Biol Med. 133:254-261. doi:10.1016/j.freeradbiomed.2018.07.001.

Sasaki, K., and E. R. Norwitz. 2011. Gonadotropin-releasing hormone/gonadotropinreleasing hormone receptor signaling in the placenta. Curr Opin Endocrinol Diabetes Obes. 18:401-408. doi:10.1097/MED.0b013e32834cd3b0. 
Savic, I., A. Garcia-Falgueras, and D. Swaab. 2010. Sexual differentiation of the human brain in relation to gender identity and sexual orientation. In: Sex Differences in the Human Brain, their Underpinnings and Implications. Vol. 186. Elsevier Enhanced Reader. p. 41-62. Available from: https://reader.elsevier.com/reader/sd/pii/B978044453630300004X?token=2BFFF D865443ABAE37707980F98FDCEC96AB5D1100490CC925AF4FEE8F30C6E E4A930147C50825FAD269304CFC583DF6

Schachtschneider, K. M., L. B. Schook, J. J. Meudt, D. Shanmuganayagam, J. A. Zoller, A. Haghani, C. Z. Li, J. Zhang, A. Yang, K. Raj, and S. Horvath. 2020. Epigenetic clock and DNA methylation analysis of porcine models of aging and obesity. bioRxiv. 2020.09.29.319509. doi:10.1101/2020.09.29.319509.

Schachtschneider, K. M., O. Madsen, C. Park, L. A. Rund, M. A. M. Groenen, and L. B. Schook. 2015. Adult porcine genome-wide DNA methylation patterns support pigs as a biomedical model. BMC Genomics. 16:743-760. doi:10.1186/s12864015-1938-x.

Semple, B. D., K. Blomgren, K. Gimlin, D. M. Ferriero, and L. J. Noble-Haeusslein. 2013. Brain development in rodents and humans: Identifying benchmarks of maturation and vulnerability to injury across species. Prog Neurobiol. 106:1-16. doi:10.1016/j.pneurobio.2013.04.001.

Shallie, P. D., and T. Naicker. 2019. The placenta as a window to the brain: A review on the role of placental markers in prenatal programming of neurodevelopment. Int $\mathbf{J}$ Dev Neurosci. 73:41-49. doi:10.1016/j.ijdevneu.2019.01.003. 
Sharp, A. J., E. Stathaki, E. Migliavacca, M. Brahmachary, S. B. Montgomery, Y. Dupre, and S. E. Antonarakis. 2011. DNA methylation profiles of human active and inactive X chromosomes. Genome Res. 21:1592-1600. doi:10.1101/gr.112680.110.

Shiau, S., L. Wang, H. Liu, Y. Zheng, A. Drong, B. T. Joyce, J. Wang, W. Li, J. Leng, Y. Shen, R. Gao, G. Hu, L. Hou, and A. A. Baccarelli. 2020. Prenatal gestational diabetes mellitus exposure and accelerated offspring DNA methylation age in early childhood. Epigenetics. 16:186-195. doi:10.1080/15592294.2020.1790924.

Sidorenko, J., I. Kassam, K. E. Kemper, J. Zeng, L. R. Lloyd-Jones, G. W. Montgomery, G. Gibson, A. Metspalu, T. Esko, J. Yang, A. F. McRae, and P. M. Visscher. 2019. The effect of X-linked dosage compensation on complex trait variation. Nat Commun. 10:3009. doi:10.1038/s41467-019-10598-y.

Sierra, M. I., A. F. Fernández, and M. F. Fraga. 2015. Epigenetics of aging. Curr Genomics. 16:435-440. doi:10.2174/1389202916666150817203459.

Simpkin, A. J., G. Hemani, M. Suderman, T. R. Gaunt, O. Lyttleton, W. L. Mcardle, S. M. Ring, G. C. Sharp, K. Tilling, S. Horvath, S. Kunze, A. Peters, M. Waldenberger, C. Ward-Caviness, E. A. Nohr, T. I. A. Sørensen, C. L. Relton, and G. D. Smith. 2016. Prenatal and early life influences on epigenetic age in children: a study of mother-offspring pairs from two cohort studies. Hum Mol Genet. 25:191-201. doi:10.1093/hmg/ddv456. 
Singh, S. K., M. N. Kagalwala, J. Parker-Thornburg, H. Adams, and S. Majumder. 2008. REST maintains self-renewal and pluripotency of embryonic stem cells. Nature. 453:223-227. doi:10.1038/nature06863.

Singhal, R. P., L. L. Mays-Hoopes, and G. L. Eichhorn. 1987. DNA methylation in aging of mice. Mech Ageing Dev. 41:199-210. doi:10.1016/0047-6374(87)90040-6.

Sjöstedt, E., W. Zhong, L. Fagerberg, M. Karlsson, N. Mitsios, C. Adori, P. Oksvold, F. Edfors, A. Limiszewska, F. Hikmet, J. Huang, Y. Du, L. Lin, Z. Dong, L. Yang, X. Liu, H. Jiang, X. Xu, J. Wang, H. Yang, L. Bolund, A. Mardinoglu, C. Zhang, K. von Feilitzen, C. Lindskog, F. Pontén, Y. Luo, T. Hökfelt, M. Uhlén, and J. Mulder. 2020. An atlas of the protein-coding genes in the human, pig, and mouse brain. Science. 367:eaay5947. doi:10.1126/science.aay5947.

Soares, M. J., K. M. Varberg, and K. Iqbal. 2018. Hemochorial placentation: development, function, and adaptations. Biol. Reprod. 99:196-211. doi:10.1093/biolre/ioy049.

Soede, N. M., P. Langendijk, and B. Kemp. 2011. Reproductive cycles in pigs. Anim Reprod Sci. 124:251-258. doi:10.1016/j.anireprosci.2011.02.025.

Somel, M., S. Guo, N. Fu, Z. Yan, H. Y. Hu, Y. Xu, Y. Yuan, Z. Ning, Y. Hu, C. Menzel, H. Hu, M. Lachmann, R. Zeng, W. Chen, and P. Khaitovich. 2010. MicroRNA, mRNA, and protein expression link development and aging in human and macaque brain. Genome Res. 20:1207-1218. doi:10.1101/gr.106849.110. 
Sookoian, S., T. F. Gianotti, A. L. Burgueño, and C. J. Pirola. 2013. Fetal metabolic programming and epigenetic modifications: a systems biology approach. Pediatr. Res. 73:531-542. doi:10.1038/pr.2013.2.

Spiers, H., E. Hannon, L. C. Schalkwyk, R. Smith, C. C. Y. Wong, M. C. O’Donovan, N. J. Bray, and J. Mill. 2015. Methylomic trajectories across human fetal brain development. Genome Res. 25:338-352. doi:10.1101/gr.180273.114.

Starks, R. R., A. Biswas, A. Jain, and G. Tuteja. 2019. Combined analysis of dissimilar promoter accessibility and gene expression profiles identifies tissue-specific genes and actively repressed networks. Epigenetics Chromatin. 12:16. doi:10.1186/s13072-019-0260-2.

Stenhouse, C., C. O. Hogg, and C. J. Ashworth. 2019. Novel relationships between porcine fetal size, sex, and endometrial angiogenesis. Biol Reprod. 101:112-125. doi:10.1093/biolre/ioz068.

Steuer, R., J. Kurths, C. O. Daub, J. Weise, and J. Selbig. 2002. The mutual information: Detecting and evaluating dependencies between variables. Bioinformatics. 18:S231-S240. doi:10.1093/bioinformatics/18.suppl_2.S231.

St-Pierre, M. V., T. Stallmach, A. Freimoser Grundschober, J.-F. Dufour, M. A. Serrano, J. J. G. Marin, Y. Sugiyama, and P. J. Meier. 2004. Temporal expression profiles of organic anion transport proteins in placenta and fetal liver of the rat. Am J Physiol Regul Integr Comp Physiol. 287:R1505-1516. doi:10.1152/ajpregu.00279.2003. 
Stubbs, T. M., M. J. Bonder, A.-K. Stark, F. Krueger, D. Bolland, G. Butcher, T. Chandra, S. J. Clark, A. Corcoran, M. Eckersley-Maslin, L. Field, U. C. Frising, C. Gilbert, J. Guedes, I. Hernando-Herraez, J. Houseley, F. Kemp, A. MacQueen, F. von Meyenn, K. Okkenhaug, W. Reik, M. Rhoades, M. J. C. Santbergen, A.-K. Stark, M. Stebegg, T. M. Stubbs, M. Veldhoen, F. von Meyenn, O. Stegle, W. Reik, and BI Ageing Clock Team. 2017. Multi-tissue DNA methylation age predictor in mouse. Genome Biol. 18:68-81. doi:10.1186/s13059-017-1203-5.

Sugiyama, D., H. Kusuhara, H. Taniguchi, S. Ishikawa, Y. Nozaki, H. Aburatani, and Y. Sugiyama. 2003. Functional characterization of rat brain-specific organic anion transporter (Oatp14) at the blood-brain barrier: high affinity transporter for thyroxine. J Biol Chem. 278:43489-43495. doi:10.1074/jbc.M306933200.

Swindle, M. M., A. Makin, A. J. Herron, F. J. Clubb, and K. S. Frazier. 2012. Swine as models in biomedical research and toxicology testing. Vet Pathol. 49:344-356. doi:10.1177/0300985811402846.

Tacutu, R., D. Thornton, E. Johnson, A. Budovsky, D. Barardo, T. Craig, E. Diana, G. Lehmann, D. Toren, J. Wang, V. E. Fraifeld, and J. P. de Magalhães. 2018. Human ageing genomic resources: new and updated databases. Nucleic Acids Res. 46:D1083-D1090. doi:10.1093/nar/gkx1042.

Tallinen, T., J. Y. Chung, J. S. Biggins, and L. Mahadevan. 2014. Gyrification from constrained cortical expansion. PNAS. 111:12667-12672. doi:10.1073/pnas.1406015111. 
Tawa, R., T. Ono, A. Kurishita, S. Okada, and S. Hirose. 1990. Changes of DNA methylation level during pre-and postnatal periods in mice. Differentiation. 45:44-48. doi:10.1111/j.1432-0436.1990.tb00455.x.

Taylor, H. S., G. B. Vanden Heuvel, and P. Igarashi. 1997. A conserved hox axis in the mouse and human female reproductive system: Late establishment and persistent adult expression of the Hoxa cluster genes. Biol. Reprod. 57:1338-1345. doi:10.1095/biolreprod57.6.1338.

Tekola-Ayele, F., T. Workalemahu, G. Gorfu, D. Shrestha, B. Tycko, R. Wapner, C. Zhang, and G. Louis. 2019. Sex differences in the associations of placental epigenetic aging with fetal growth. Aging. 11:5412-5432. doi:10.18632/aging.102124.

Terry, M. B., L. Delgado-Cruzata, N. Vin-Raviv, H. C. Wu, and R. M. Santella. 2011. DNA methylation in white blood cells: association with risk factors in epidemiologic studies. Epigenetics. 6:828-837. doi:10.4161/epi.6.7.16500.

The UniProt Consortium. 2019. UniProt: a worldwide hub of protein knowledge. Nucleic Acids Res. 47:D506-D515. doi:10.1093/nar/gky1049.

Tzika, E., T. Dreker, and A. Imhof. 2018. Epigenetics and metabolism in health and disease. Front. Genet. 9:361-368. doi:10.3389/fgene.2018.00361.

Vassoler, F. M., S. L. White, H. D. Schmidt, G. Sadri-Vakili, and R. C. Pierce. 2013. Epigenetic inheritance of a cocaine resistance phenotype. Nat Neurosci. 16:42-47. doi:10.1038/nn.3280. 
Villadsen, J., H. D. Hansen, L. M. Jørgensen, S. H. Keller, F. L. Andersen, I. N. Petersen, G. M. Knudsen, and C. Svarer. 2018. Automatic delineation of brain regions on MRI and PET images from the pig. J Neurosci Methods. 294:51-58. doi:10.1016/j.jneumeth.2017.11.008.

vom Saal, F. S. 1989. Sexual differentiation in litter-bearing mammals: influence of sex of adjacent fetuses in utero. J Anim Sci. 67:1824-1840. doi:10.2527/jas1989.6771824x.

Wadhwa, P. D., C. Buss, S. Entringer, and J. M. Swanson. 2009. Developmental origins of health and disease: brief history of the approach and current focus on epigenetic mechanisms. Semin Reprod Med. 27:358-368. doi:10.1055/s-00291237424.

Wagner, A. 2005. Energy constraints on the evolution of gene expression. Mol Biol Evol. 22:1365-1374. doi:10.1093/molbev/msi126.

Walentin, K., C. Hinze, M. Werth, N. Haase, S. Varma, R. Morell, A. Aue, E. Pötschke, D. Warburton, A. Qiu, J. Barasch, B. Purfürst, C. Dieterich, E. Popova, M. Bader, R. Dechend, A. C. Staff, Z. Y. Yurtdas, E. Kilic, and K. M. Schmidt-Ott. 2015. A Grhl2-dependent gene network controls trophoblast branching morphogenesis. Development. 142:1125-1136. doi:10.1242/dev.113829.

Walton, E., J. Hass, J. Liu, J. L. Roffman, F. Bernardoni, V. Roessner, M. Kirsch, G. Schackert, V. Calhoun, and S. Ehrlich. 2016. Correspondence of DNA methylation between blood and brain tissue and its application to schizophrenia research. Schizophr Bull. 42:406-414. doi:10.1093/schbul/sbv074. 
Wang, P., Y. Song, H. Zhong, S. Lin, X. Zhang, J. Li, L. Che, B. Feng, Y. Lin, S. Xu, Y. Zhuo, D. Wu, D. G. Burrin, and Z. Fang. 2019. Transcriptome profiling of placenta through pregnancy reveals dysregulation of bile acids transport and detoxification function. Int J Mol Sci. 20. doi:10.3390/ijms20174099.

Wang, X., and H. N. Kadarmideen. 2019. An epigenome-wide DNA methylation map of testis in pigs for study of complex traits. Front Genet. 10:405-417. doi:10.3389/fgene.2019.00405.

Wang, X., K. C. Douglas, J. L. Vandeberg, A. G. Clark, and P. B. Samollow. 2014. Chromosome-wide profiling of X-chromosome inactivation and epigenetic states in fetal brain and placenta of the opossum, Monodelphis domestica. Genome Res. 24:70-83. doi:10.1101/gr.161919.113.

Wang, X., S. Khatri, R. Broaddus, Z. Wang, and S. M. Hawkins. 2016. Deletion of Arid1a in reproductive tract mesenchymal cells reduces fertility in female mice. Biol Reprod. 94:93. doi:10.1095/biolreprod.115.133637.

Weber, A. P. M., K. L. Weber, K. Carr, C. Wilkerson, and J. B. Ohlrogge. 2007. Sampling the Arabidopsis transcriptome with massively parallel pyrosequencing. Plant Physiol. 144:32-42. doi:10.1104/pp.107.096677.

Wells, K. D., and R. S. Prather. 2017. Genome-editing technologies to improve research, reproduction, and production in pigs. Mol Reprod Dev. 84:1012-1017. doi:10.1002/mrd.22812. 
Wierenga, L. M., M. G. N. Bos, F. van Rossenberg, and E. A. Crone. 2019. Sex effects on development of brain structure and executive functions: Greater variance than mean effects. J. Cogn. Neurosci. 31:730-753. doi:10.1162/jocn_a_01375.

Wilms, I., and C. Croux. 2015. Sparse canonical correlation analysis from a predictive point of view. Biom J. 57:834-851. doi:10.1002/bimj.201400226.

Wilson, V. L., R. A. Smith, S. Ma, and R. G. Cutler. 1987. Genomic 5methyldeoxycytidine decreases with age. J. Biol. Chem. 262:9948-9951.

Winter, J. D., S. Dorner, J. Lukovic, J. A. Fisher, K. S. St. Lawrence, and A. Kassner. 2011. Noninvasive MRI measures of microstructural and cerebrovascular changes during normal swine brain development. Pediatr. Res. 69:418-424. doi:10.1203/PDR.0b013e3182110f7e.

Withington, S. L., A. N. Scott, D. N. Saunders, K. Lopes Floro, J. I. Preis, J. Michalicek, K. Maclean, D. B. Sparrow, J. P. M. Barbera, and S. L. Dunwoodie. 2006. Loss of Cited 2 affects trophoblast formation and vascularization of the mouse placenta. Developmental Biology. 294:67-82. doi:10.1016/j.ydbio.2006.02.025.

Wu, H., B. Caffo, H. A. Jaffee, R. A. Irizarry, and A. P. Feinberg. 2010. Redefining CpG islands using hidden Markov models. Biostatistics. 11:499-514. doi:10.1093/biostatistics/kxq005.

Xu, J., and C. M. Disteche. 2006. Sex differences in brain expression of X-and Y-linked genes. Brain Res. 1126:50-55. doi:10.1016/j.brainres.2006.08.049. 
Xuan Lin, Q. X., S. Sian, O. An, D. Thieffry, S. Jha, and T. Benoukraf. 2019. MethMotif: an integrative cell specific database of transcription factor binding motifs coupled with DNA methylation profiles. Nucleic Acids Res. 47:D145-D154. doi:10.1093/nar/gky1005.

Yates, A. D., P. Achuthan, W. Akanni, James Allen, Jamie Allen, J. Alvarez-Jarreta, M. R. Amode, I. M. Armean, A. G. Azov, R. Bennett, J. Bhai, K. Billis, S. Boddu, J. C. Marugán, C. Cummins, C. Davidson, K. Dodiya, R. Fatima, A. Gall, C. G. Giron, L. Gil, T. Grego, L. Haggerty, E. Haskell, T. Hourlier, O. G. Izuogu, S. H. Janacek, T. Juettemann, M. Kay, I. Lavidas, T. Le, D. Lemos, J. G. Martinez, T. Maurel, M. McDowall, A. McMahon, S. Mohanan, B. Moore, M. Nuhn, D. N. Oheh, A. Parker, A. Parton, M. Patricio, M. P. Sakthivel, A. I. Abdul Salam, B. M. Schmitt, H. Schuilenburg, D. Sheppard, M. Sycheva, M. Szuba, K. Taylor, A. Thormann, G. Threadgold, A. Vullo, B. Walts, A. Winterbottom, A. Zadissa, M. Chakiachvili, B. Flint, A. Frankish, S. E. Hunt, G. IIsley, M. Kostadima, N. Langridge, J. E. Loveland, F. J. Martin, J. Morales, J. M. Mudge, M. Muffato, E. Perry, M. Ruffier, S. J. Trevanion, F. Cunningham, K. L. Howe, D. R. Zerbino, and P. Flicek. 2020. Ensembl. Nucleic Acids Res. 48:D682-D688. doi:10.1093/nar/gkz966.

Yoon, H. J., J. H. Kim, T. Y. Jeon, S.-Y. Yoo, and H. Eo. 2014. Devastating metabolic brain disorders of newborns and young infants. RadioGraphics. 34:1257-1272. doi:10.1148/rg.345130095. 
Youness, A., C.-H. Miquel, and J.-C. Guéry. 2021. Escape from X chromosome inactivation and the female predominance in autoimmune diseases. Int J Mol Sci. 22:1114. doi:10.3390/ijms22031114.

Zak, L. J., A. H. Gaustad, A. Bolarin, M. L. W. J. Broekhuijse, G. A. Walling, and E. F. Knol. 2017. Genetic control of complex traits, with a focus on reproduction in pigs. Mol Reprod Dev. 84:1004-1011. doi:10.1002/mrd.22875.

Zeltser, L. M., and R. L. Leibel. 2011. Roles of the placenta in fetal brain development. PNAS. 108:15667-15668. doi:10.1073/pnas.1112239108.

Zhang, W., and H. T. Liu. 2002. MAPK signal pathways in the regulation of cell proliferation in mammalian cells. Cell Research. 12:9-18. doi:10.1038/sj.cr.7290105.

Zhu, J., F. Chen, L. Luo, W. Wu, J. Dai, J. Zhong, X. Lin, C. Chai, P. Ding, L. Liang, S. Wang, X. Ding, Y. Chen, H. Wang, J. Qiu, F. Wang, C. Sun, Y. Zeng, J. Fang, X. Jiang, P. Liu, G. Tang, X. Qiu, X. Zhang, Y. Ruan, S. Jiang, J. Li, S. Zhu, X. Xu, F. Li, Z. Liu, G. Cao, and D. Chen. 2021. Single-cell atlas of domestic pig cerebral cortex and hypothalamus. Science Bulletin. doi:10.1016/j.scib.2021.04.002.

Zullo, J. M., D. Drake, L. Aron, P. O’Hern, S. C. Dhamne, N. Davidsohn, C.-A. Mao, W. H. Klein, A. Rotenberg, D. A. Bennett, G. M. Church, M. P. Colaiácovo, and B. A. Yankner. 2019. Regulation of lifespan by neural excitation and REST. Nature. 574:359-364. doi:10.1038/s41586-019-1647-8. 